PUPT-2237

ITEP-TH/22-07

\title{
Towards an Explicit Model of D-brane Inflation
}

\author{
Daniel Baumann, ${ }^{1}$ Anatoly Dymarsky, ${ }^{1}$ \\ Igor R. Klebanov, ${ }^{1,2}$ and Liam McAllister ${ }^{1}$ \\ ${ }^{1}$ Department of Physics, Princeton University, Princeton, NJ 08544 \\ ${ }^{2}$ Princeton Center for Theoretical Physics, Princeton University, Princeton, NJ 08544
}

\begin{abstract}
We present a detailed analysis of an explicit model of warped D-brane inflation, incorporating the effects of moduli stabilization. We consider the potential for D3-brane motion in a warped conifold background that includes fluxes and holomorphically-embedded D7-branes involved in moduli stabilization. Although the D7-branes significantly modify the inflaton potential, they do not correct the quadratic term in the potential, and hence do not cause a uniform change in the slow-roll parameter $\eta$. Nevertheless, we present a simple example based on the Kuperstein embedding of D7-branes, $z_{1}=$ constant, in which the potential can be fine-tuned to be sufficiently flat for inflation. To derive this result, it is essential to incorporate the fact that the compactification volume changes slightly as the D3-brane moves. We stress that the compactification geometry dictates certain relationships among the parameters in the inflaton Lagrangian, and these microscopic constraints impose severe restrictions on the space of possible models. We note that the shape of the final inflaton potential differs from projections given in earlier studies: in configurations where inflation occurs, it does so near an inflection point. Finally, we comment on the difficulty of making precise cosmological predictions in this scenario. This is the companion paper to [1].
\end{abstract}

October 22, 2018 


\section{Contents}

1 Introduction $\quad 2$

2 D3-brane Potential in Warped Backgrounds 5

2.1 The compactification . . . . . . . . . . . . . . 5 5

2.2 D3-brane potential from moduli stabilization . . . . . . . . . . . 7

2.3 Potential in the warped conifold throat . . . . . . . . . . . . . . . 9

3 Case Study: Kuperstein Embedding $\quad 10$

3.1 Multi-field potential . . . . . . . . . . . . . . . 11

3.2 Effective single-field potential . . . . . . . . . . . . . . . . 13

4 Search for an Inflationary Trajectory $\quad 15$

4.1 Analytic considerations . . . . . . . . . . . . . . . 16

4.2 Parameters and microscopic constraints . . . . . . . . . . . . . 17

4.3 Numerical results . . . . . . . . . . . . . . . . . . . . . . . . . . . . . . . 19

4.4 Phenomenological model: cubic inflation . . . . . . . . . . . . . . 21

5 Comments on Other Embeddings $\quad 25$

6 Discussion $\quad 29$

7 Conclusions $\quad 31$

$\begin{array}{ll}\text { A Background Geometry } & 35\end{array}$

$\begin{array}{ll}\text { B Dimensional Reduction } & 37\end{array}$

C Stability in the Angular Directions $\quad 44$

D Stabilization of the Volume $\quad 54$

$\begin{array}{llc}\text { E Effects of Induced Magnetic Fields } & 58\end{array}$

F Symbols Used in the Paper $\quad 62$ 


\section{Introduction}

Inflation [2] provides an explanation for certain observed properties of our universe, from large-scale isotropy and homogeneity to the small-scale anisotropies in the cosmic microwave background (CMB). Recent observational advances [3] have begun to constrain the space of inflationary models, and in the next decade it should be possible to exclude a large fraction of current models. This is a striking opportunity to extract clues about physics at extremely high energy scales. At the same time, there is a pressing need to develop a more fundamental understanding of the physics of inflation. String theory, as a quantum theory of Planck-scale physics, is a promising candidate for the fundamental theory underlying the inflationary paradigm.

In this paper we will be concerned with making progress towards an explicit model of inflation in string theory, by which we mean a model in which the fields and parameters in the low-energy Lagrangian are derived from the data of a string compactification. In such a scenario, questions about the structure of the inflaton potential can be resolved by concrete string theory computations. This should be contrasted with string-inspired models constructed directly in effective field theory, for which naturalness is the final arbiter of the form of the potential. We will not quite reach the ambitious goal of an entirely explicit model of inflation derived from string theory, and indeed one main point of this paper is that truly explicit models of string inflation can be rather intricate, involving multiple nontrivial microscopic constraints that are surprising from the low-energy perspective.

We will work in the setting of D-brane inflation $[4,5,6]$, a promising framework that has attracted considerable interest, but in which concrete, working models remain scarce. ${ }^{1}$ In this approach to string inflation the inflaton field is identified with the location of a mobile D-brane, usually a D3-brane, in the compactification manifold. The proposal [4] is that a weak force on this brane, e.g. from a distant antibrane, could give rise to a relatively flat inflaton potential. In practice, this is difficult to achieve: the Coulomb potential of a braneantibrane pair in an unwarped compactification is generically too steep for inflation. In [6], it was observed that a brane-antibrane pair separated along a warped direction enjoys a much flatter potential. At the same time, it was recognized that moduli stabilization, which is essential for a successful cosmological model in string theory, gives rise to corrections to the inflaton potential. Some of these corrections arise from complicated properties of the compactification and have been computed only recently $[9] .^{2}$

The attitude taken in most of the literature on the subject (see e.g. $[6,13,8]$ ) is that because of the vast number and complexity of string vacua, in some nonzero fraction of them it should be the case that the corrections to the inflaton potential cancel to high precision,

\footnotetext{
${ }^{1}$ For other approaches to string inflation, see e.g. [7], as well as [8] and references therein.

${ }^{2}$ For important earlier work, see $[10,11,12]$.
} 
leaving a suitable inflationary model. However, there is no guarantee that this hope can be realized: for example, the correction terms may invariably have the same sign so that no cancellation can ever occur. Moreover, the precise nature of the cancellation will affect the detailed predictions of the model. In this paper we will systematically address the question of whether or not this hope of fine-tuned cancellation can in fact be realized. The new ingredient that makes this investigation possible is our recent result, obtained in collaboration with J. Maldacena and A. Murugan [9], for the one-loop correction to the volume-stabilizing nonperturbative superpotential. This effect is due to the interaction $[11,12]$ between the inflationary D3-brane and the moduli-stabilizing wrapped branes, i.e. D7-branes wrapping a four-cycle within the Calabi-Yau threefold. ${ }^{3}$ The location of these wrapped branes therefore becomes a crucial parameter in the D3-brane potential.

With this theoretical input we ask whether the known correction terms to the inflaton potential can indeed cancel for specific values of the microphysical input parameters. To investigate this, we study D3-brane motion in warped conifold backgrounds [14, 15] for a large class of wrapped brane embeddings. By identifying radial trajectories that are stable in the angular directions, we integrate out the angular degrees of freedom and arrive at an effective two-field potential for the inflaton - corresponding to the radial direction in the throat - and the compactification volume. Because we work in a framework with explicitly stabilized moduli, the compactification volume has a positive mass-squared. However, this mass is not so large that the volume remains fixed at a single value during inflation. Instead, the minimum of the potential for the volume shifts as the D3-brane moves: the compact space shrinks slightly as the D3-brane falls down the throat. Properly incorporating this phenomenon leads to a nontrivial change in the effective single-field inflaton potential. Thus, we find that an approximation that keeps the volume fixed at its KKLT [16] minimum during inflation is not sufficiently accurate for a D-brane inflation model. Our improved approximation is that the volume changes adiabatically, remaining in an inflaton-dependent minimum, as the D3-brane moves.

Equipped with the effective single-field potential, we ask whether the trajectories that are stable in the angular directions can enjoy flat potentials. For the large class of holomorphic wrapped brane embeddings described in [17], we find trajectories that are too steep to permit inflation, even with an arbitrary amount of fine-tuning of the compactification parameters: the functional form of the leading corrections to the potential makes fine-tuning impossible. (Our conclusions are consistent with those reached in [18] for the special case of the Ouyang embedding [19].) This illustrates a key virtue of the explicit, top-down approach: by direct computation we can refute the very reasonable expectation that fine-tuning is generically possible.

\footnotetext{
${ }^{3}$ Alternatively, one could consider Euclidean D3-branes wrapping this four-cycle. Their effect on the nonperturbative superpotential is very similar to that of the D7-branes.
} 
Undeterred by this no-go result for a wide class of D3-brane trajectories, we devote a large portion of this paper to showing that in a particularly simple and symmetric embedding due to Kuperstein [20], the stable trajectory is not necessarily steep. We then establish that for fine-tuned values of the microphysical parameters, a D3-brane following this stable trajectory leads to sustained slow-roll inflation near an inflection point of the potential. Finally, we derive nontrivial constraints, due to the consistency of the embedding of the warped throat in a flux compactification, that relate the microscopic parameters of the inflaton Lagrangian. These constraints sharply restrict the parameter space of the model, and in fact exclude most, but not all, of the parameter space that permits sustained inflation.

Our result provides evidence for the existence of successful warped D-brane inflation models based on concrete microscopic data of a flux compactification. However, we emphasize that inflation is non-generic in this class of D-brane models. In fact, because of the geometric constraints, it is surprisingly difficult, though not impossible, to achieve inflation.

The outline of this paper is as follows: in $\S 2$ we briefly review D-brane inflation in warped backgrounds. We then provide results for the complete D3-brane potential in a warped throat region of the compactification. This includes an important correction to the volume-stabilizing nonperturbative superpotential first computed in [9]. In $\S 3$ we present a detailed study of a simple example, the case of the Kuperstein embedding [20] of the wrapped branes. By integrating out the complex Kähler modulus and the angular positions of the D3-brane, we derive effective single-field potentials for different brane trajectories. In $\S 4$ we then prove the existence of a stable inflationary trajectory, but also discuss important constraints on microscopic parameters that make inflation challenging to achieve. In $\S 5$ we comment on generalizations to other embeddings. We then take the opportunity, in $\S 6$, to make some general remarks about the problem of relating string compactification data to the low energy Lagrangian. We conclude in $\S 7$.

In order to make the body of the paper more readable, we have relegated a number of more technical results to a series of appendices. Although most of these results are new, they could be omitted on a first reading. Appendix A gives details of the conifold geometry and of the supergravity F-term potential. In Appendix B we dimensionally reduce the ten-dimensional string action and derive microscopic constraints on the inflaton field range and the warped four-cycle volume. In particular, we explain how to generalize the field range bound of [21] to a compactification with a nontrivial breathing mode. We also derive a new constraint that relates the field range and the volume of the wrapped four-cycle. Appendix $\mathrm{C}$ provides more technical aspects of the proof that the inflationary trajectory is stable against angular fluctuations. In Appendix D we derive the dependence of the compactification volume on the D3-brane position. This is an important improvement on the typical approach of keeping the volume fixed as the D3-brane moves. Appendix E addresses the concern that induced magnetic fields on the D7-branes could affect the D3-brane potential. Finally, Appendix F 
collects the definitions of all variables used in this work.

A condensed presentation of some of the key results of this paper has appeared in [1].

\section{D3-brane Potential in Warped Backgrounds}

In warped brane-antibrane inflation [6], one considers the motion of a D3-brane towards an anti-D3-brane that sits at the bottom of a warped throat region of a stabilized flux compactification. The inflaton field is the separation between the brane and the antibrane.

\subsection{The compactification}

Our setting is a flux compactification [22] (see [23] for a review) of type IIB string theory on an orientifold of a Calabi-Yau threefold (or an F-theory compactification on a Calabi-Yau fourfold). We suppose that the fluxes are chosen so that the internal space has a warped throat region. As a simple, concrete example of this local geometry, we consider the warped deformed conifold [14]. The deformed conifold is a subspace of complex dimension three in $\mathbb{C}^{4}$ defined by the constraint equation

$$
\sum_{i=1}^{4} z_{i}^{2}=\varepsilon^{2}
$$

where $\left\{z_{i}, i=1,2,3,4\right\}$ are complex coordinates in $\mathbb{C}^{4}$. The deformation parameter $\varepsilon$ can be made real by an appropriate phase rotation. The region relevant to our modeling of D-brane inflation lies far from the bottom of the throat, where the right hand side of (2.1) can be ignored and the metric of the deformed conifold is well-approximated by that of the singular conifold,

$$
\mathrm{d} s_{6}^{2}=\mathrm{d} \hat{r}^{2}+\hat{r}^{2} \mathrm{~d} s_{T^{1,1}}^{2},
$$

where $\mathrm{d} s_{T^{1,1}}^{2}$ is the metric of the Einstein manifold $T^{1,1}$, the base of the cone (see Appendix A). This Calabi-Yau metric is obtained from the Kähler potential [24]

$$
k=\frac{3}{2}\left(\sum_{i=1}^{4}\left|z_{i}\right|^{2}\right)^{2 / 3}=\frac{3}{2} r^{2}=\hat{r}^{2} .
$$

The warping is achieved by turning on $M$ units of $F_{3}$ flux through the A-cycle of the deformed conifold (the three-sphere at the bottom) and $-K$ units of $H_{3}$ flux through the dual B-cycle. The resulting warped deformed conifold background is given in [14, 25]. If $\hat{r}_{\mathrm{UV}}$ is the maximum radial coordinate where the throat is glued into a compact manifold, then 
for $\varepsilon^{2 / 3} \ll \hat{r} \ll \hat{r}_{\mathrm{UV}}$ the background is well-approximated by the warped conifold [15]

$$
\mathrm{d} s_{10}^{2}=h^{-1 / 2}(\hat{r}) \mathrm{d} s_{4}^{2}+h^{1 / 2}(\hat{r}) \mathrm{d} s_{6}^{2},
$$

with the warp factor $[15,25]$

$$
h(\hat{r})=\frac{L^{4}}{\hat{r}^{4}} \ln \frac{\hat{r}}{\varepsilon^{2 / 3}}, \quad L^{4}=\frac{81}{8}\left(g_{s} M \alpha^{\prime}\right)^{2} .
$$

We also have $[22,25]$

$$
\ln \frac{\hat{r}_{\mathrm{UV}}}{\varepsilon^{2 / 3}} \approx \frac{2 \pi K}{3 g_{s} M} .
$$

The scale of supersymmetry breaking associated with an anti-D3-brane at the bottom of the throat is $D=2 T_{3} h_{0}^{-1}$, where $h_{0}$ is the warp factor there. The approximation (2.5) is not accurate enough to determine the warp factor at the bottom of the throat; its value is $[14,25]$

$$
h_{0}=a_{0}\left(g_{s} M \alpha^{\prime}\right)^{2} 2^{2 / 3} \varepsilon^{-8 / 3}, \quad a_{0} \approx 0.71805,
$$

which is approximately $h_{0} \approx e^{8 \pi K / 3 g_{s} M}[22]$.

Following [16], we require that all the closed string moduli are stabilized, ${ }^{4}$ by a combination of fluxes and nonperturbative effects. Each nonperturbative effect may arise either from Euclidean D3-branes wrapping a four-cycle, or from strong gauge dynamics, such as gaugino condensation, on a stack of $n>1$ D7-branes wrapping a four-cycle. Finally, as in [9], we require that at least one of the four-cycles bearing nonperturbative effects descends a finite distance into the warped throat. For simplicity of presentation we will refer to the nonperturbative effects on this cycle as originating on D7-branes, but all our results apply equally well to the case in which Euclidean D3-branes are responsible for this effect.

An embedding is specified by the number $n=N_{D 7}>1$ of D7-branes and the minimal radial coordinate $r_{\mu}$ reached by the D7-branes. The stabilization of $r_{\mu}$ is a potentially confusing issue, so we pause to explain it. In the construction [20] of supersymmetric wrapped D7-branes in the noncompact $\mathrm{KS}$ throat, $r_{\mu}$ is a free parameter. One might therefore think that the wrapped D7-branes are not stabilized, and that there is a massless field corresponding to changes in $r_{\mu}$. However, in the F-theory picture, $r_{\mu}$ is determined by the complex structure of the fourfold. For generic choices of four-form fluxes, this complex structure is entirely fixed [26] (see also [27]), just as the threefold complex structure is fixed in type IIB compactifications with generic three-form fluxes [22]. Moreover, the scale of the associated mass terms (see e.g. [28]), $m_{\text {flux }} \sim \frac{\alpha^{\prime}}{\sqrt{V_{6}}}$, with $V_{6}$ the volume of the compact space, is considerably higher than the (warped) energy scale associated with the brane-antibrane pair

\footnotetext{
${ }^{4}$ This condition is necessary for a realistic model, and amounts to a nontrivial selection criterion on the space of compactifications.
} 


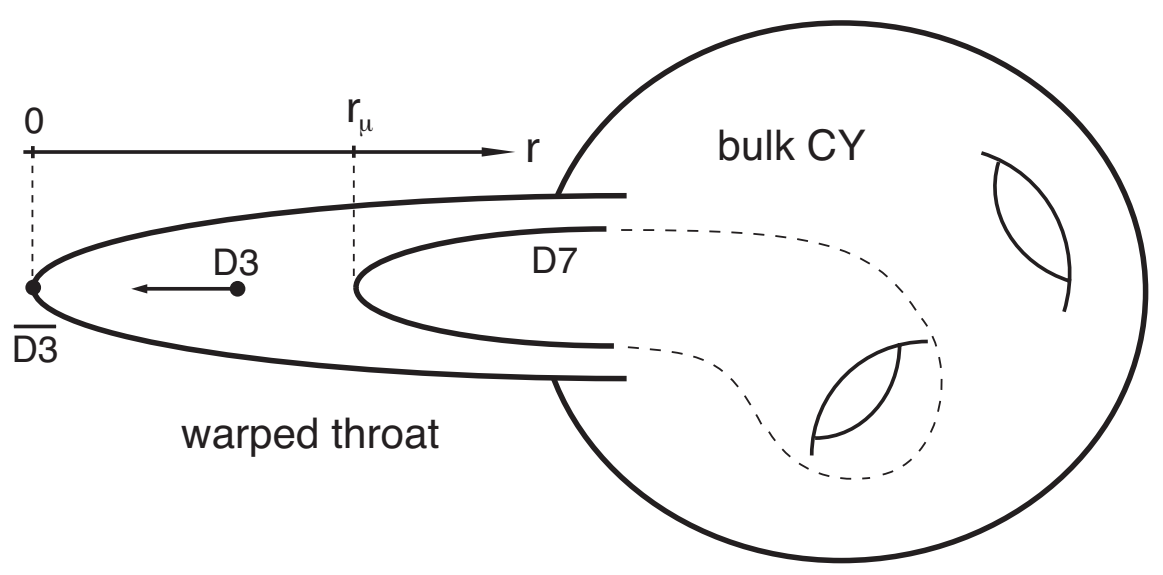

Figure 1: Cartoon of an embedded stack of D7-branes wrapping a four-cycle $\Sigma_{4}$, and a mobile D3-brane, in a warped throat region of a compact Calabi-Yau. The D3-brane feels a force from the D7-branes and from an anti-D3-brane at the tip of the throat.

under consideration. Hence, for our purposes the D7-brane moduli are massive enough to be ignored. Next, the stabilized value of $r_{\mu}$ is determined by the fluxes in the bulk of the fourfold. In a generic compactification the number of choices of such fluxes is vast, so we expect that for a given compactification and for any desired value $r_{\mu}^{\star}$, there exist choices of flux that fix the D7-brane to a location $r_{\mu} \approx r_{\mu}^{\star}$.

\subsection{D3-brane potential from moduli stabilization}

The effect of moduli stabilization on the D3-brane is captured by the F-term potential of $\mathcal{N}=1$ supergravity,

$$
V_{F}=e^{\kappa^{2} \mathcal{K}}\left[D_{\Sigma} W \mathcal{K}^{\Sigma \bar{\Omega}} \overline{D_{\Omega} W}-3 \kappa^{2} W \bar{W}\right], \quad \kappa^{2}=M_{P}^{-2} \equiv 8 \pi G
$$

where $\left\{Z^{\Sigma}\right\} \equiv\left\{\rho, z_{\alpha} ; \alpha=1,2,3\right\}$ and $D_{\Sigma} W=\partial_{\Sigma} W+\kappa^{2}\left(\partial_{\Sigma} \mathcal{K}\right) W$. The combined Kähler potential for the volume modulus, $\rho$, and the three open string moduli (D3-brane positions), $z_{\alpha}$, is of the form postulated by DeWolfe and Giddings $[29]^{5}$

$$
\kappa^{2} \mathcal{K}\left(\rho, \bar{\rho}, z_{\alpha}, \overline{z_{\alpha}}\right)=-3 \log \left[\rho+\bar{\rho}-\gamma k\left(z_{\alpha}, \overline{z_{\alpha}}\right)\right] \equiv-3 \log U
$$

\footnotetext{
${ }^{5}$ In [30] it was suggested that this result may receive corrections in strongly-warped scenarios. However, the proposed corrections do not affect the metric on the Kähler moduli space, and thus are irrelevant for most of the considerations presented here. However, a truly thorough search for possible effects of such corrections on our analysis must await a more complete understanding of the structure of corrections to the Kähler potential.
} 
where in general $k\left(z_{\alpha}, \overline{z_{\alpha}}\right)$ denotes the Kähler potential of the Calabi-Yau manifold. The normalization constant $\gamma$ in (2.9) is derived in Appendix B and may be expressed as

$$
\gamma \equiv \frac{\sigma_{0}}{3} \frac{T_{3}}{M_{P}^{2}}
$$

where $2 \sigma_{0} \equiv 2 \sigma_{\star}(0)=\rho_{\star}(0)+\bar{\rho}_{\star}(0)$ is the stabilized value of the Kähler modulus when the D3-brane is near the tip of the throat.

The Kähler metric $\mathcal{K}_{\Omega \bar{\Sigma}} \equiv \mathcal{K}_{, \Omega \bar{\Sigma}}$ assumes the form

$$
\mathcal{K}_{\Omega \bar{\Sigma}}=\frac{3}{\kappa^{2} U^{2}}\left(\begin{array}{c|c}
1 & -\gamma k_{\bar{\beta}} \\
\hline-\gamma k_{\alpha} & U \gamma k_{\alpha \bar{\beta}}+\gamma^{2} k_{\alpha} k_{\bar{\beta}}
\end{array}\right),
$$

where $k_{\alpha \bar{\beta}} \equiv \partial_{\alpha} \partial_{\bar{\beta}} k$ is the Calabi-Yau metric, and $k_{\alpha} \equiv k_{, \alpha}$. The problem of finding the inverse metric, $\mathcal{K}^{\Delta \bar{\Gamma}} \mathcal{K}_{\bar{\Gamma} \Omega}=\delta_{\Omega}^{\Delta}$, was solved in [18]:

$$
\mathcal{K}^{\Delta \bar{\Gamma}}=\frac{\kappa^{2} U}{3}\left(\begin{array}{c|c}
U+\gamma k_{\gamma} k^{\gamma \bar{\delta}} k_{\bar{\delta}} & k_{\gamma} k^{\gamma \bar{\beta}} \\
\hline k^{\alpha \bar{\delta}} k_{\bar{\delta}} & \frac{1}{\gamma} k^{\alpha \bar{\beta}}
\end{array}\right) .
$$

After some calculation, these results lead to the F-term potential

$$
\begin{gathered}
V_{F}\left(\rho, z_{\alpha}\right)=\frac{\kappa^{2}}{3 U^{2}}\left[\left(\rho+\bar{\rho}+\gamma\left(k_{\gamma} k^{\gamma \bar{\delta}} k_{\bar{\delta}}-k\right)\right)\left|W_{, \rho}\right|^{2}-3\left(\bar{W} W_{, \rho}+\text { c.c. }\right)\right. \\
+\underbrace{\left(k^{\alpha \bar{\delta}} k_{\bar{\delta}} \overline{W_{, \rho}} W_{, \alpha}+\text { c.c. }\right)+\frac{1}{\gamma} k^{\alpha \bar{\beta}} W_{, \alpha} \overline{W_{, \beta}}}_{\Delta V_{F}}] .
\end{gathered}
$$

The label $\Delta V_{F}$ has isolated the terms in F-term potential (2.13) that arise exclusively from the dependence of the nonperturbative superpotential on the brane position [9]. The remainder of (2.13) is the standard KKLT F-term potential [16].

The superpotential $W$ is the sum of the constant Gukov-Vafa-Witten flux superpotential [31], $W_{\text {flux }}=\int G \wedge \Omega \equiv W_{0}$, and a term from nonperturbative effects, $W_{\mathrm{np}}=A\left(z_{\alpha}\right) e^{-a \rho}$,

$$
W\left(\rho, z_{\alpha}\right)=W_{0}+A\left(z_{\alpha}\right) e^{-a \rho}, \quad a \equiv \frac{2 \pi}{n} .
$$

By a choice of phases we can arrange that $W_{0}$ is real and negative. The nonperturbative term $W_{\text {np }}$ arises either from strong gauge dynamics on a stack of $n>1$ D7-branes or from Euclidean D3-branes (with $n=1$ ). We assume that either sort of brane supersymmetrically wraps a four-cycle in the warped throat that is specified by a holomorphic embedding equation $f\left(z_{\alpha}\right)=0$. The warped volume of the four-cycle governs the magnitude of the 
nonperturbative effect, by affecting the gauge coupling on the D7-branes (equivalently, the action of Euclidean D3-branes) wrapping this four-cycle. The presence of a D3-brane gives rise to a perturbation to the warp factor, and this leads to a correction to the warped four-cycle volume. This correction depends on the D3-brane position and is responsible for the prefactor $A\left(z_{\alpha}\right)$ [12]. In [9], in collaboration with J. Maldacena and A. Murugan, we computed the D3-brane backreaction on the warped four-cycle volume. This gave the result ${ }^{6}$

$$
A\left(z_{\alpha}\right)=A_{0}\left(\frac{f\left(z_{\alpha}\right)}{f(0)}\right)^{1 / n} .
$$

See [9] for a derivation of this result, and for a more complete discussion of the setup, which we have only briefly reviewed here.

\subsection{Potential in the warped conifold throat}

In this section we apply the general formulae of the previous section to the case of a D3-brane moving in a warped deformed conifold. We will assume that both the mobile D3-brane and the fixed D7-branes are located far enough from the tip that the deformation parameter $\varepsilon$ may be neglected. If we use $z_{\alpha}=\left\{z_{1}, z_{2}, z_{3}\right\}$ as the three independent variables, the conifold constraint allows us to express $z_{4}= \pm i\left(\sum_{\alpha=1}^{3} z_{\alpha}^{2}\right)^{1 / 2}$. Using this basis, and the Kähler potential (2.3), we obtain the conifold metric

$$
\begin{aligned}
k_{\alpha \bar{\beta}} & =\frac{3}{2} \frac{\partial^{2}}{\partial z_{\alpha} \partial \overline{z_{\beta}}}\left(\sum_{\gamma=1}^{3}\left|z_{\gamma}\right|^{2}+\left|\sum_{\gamma=1}^{3} z_{\gamma}^{2}\right|\right)^{2 / 3} \\
& =\frac{1}{r}\left[\delta_{\alpha \bar{\beta}}+\frac{z_{\alpha} \overline{z_{\beta}}}{\left|z_{4}\right|^{2}}-\frac{1}{3 r^{3}}\left(z_{\alpha} \overline{z_{\beta}}+z_{\beta} \overline{z_{\alpha}}-\frac{z_{4}}{\overline{z_{4}}} \overline{z_{\alpha} z_{\beta}}-\frac{\overline{z_{4}}}{z_{4}} z_{\alpha} z_{\beta}\right)\right] .
\end{aligned}
$$

Its inverse assumes the simple form

$$
k^{\alpha \bar{\beta}}=r\left[\delta^{\alpha \bar{\beta}}+\frac{1}{2} \frac{z_{\alpha} \overline{z_{\beta}}}{r^{3}}-\frac{z_{\beta} \overline{z_{\alpha}}}{r^{3}}\right] .
$$

Expression (2.13) for the F-term potential simplifies significantly when we substitute (2.18) and note that

$$
U(\rho, r)=\rho+\bar{\rho}-\frac{3 \gamma}{2} r^{2}, \quad k^{\gamma \bar{\delta}} k_{\bar{\delta}}=\frac{3}{2} z_{\gamma}, \quad k_{\gamma} k^{\gamma \bar{\delta}} k_{\bar{\delta}}=k
$$

\footnotetext{
${ }^{6}$ The D3-brane-independent factor $A_{0}$ in (2.15) arises from threshold corrections that depend on the complex structure moduli. This quantity is not known except in special cases, but is a relatively unimportant constant in our scenario, because the complex structure moduli are stabilized by the flux background, and because, as we shall see, $A_{0}$ appears in the final potential only as an overall constant prefactor.
} 
The remaining term in the potential is the contribution of an anti-D3-brane at the tip of the conifold, including its Coulomb interaction with the mobile D3-brane [6]

$$
V_{D}(\rho, r)=\frac{D(r)}{U^{2}(\rho, r)}, \quad D(r) \equiv D\left[1-\frac{3 D}{16 \pi^{2}} \frac{1}{\left(T_{3} r^{2}\right)^{2}}\right]+D_{\text {other }} \approx D+D_{\text {other }},
$$

where $D \equiv 2 h_{0}^{-1} T_{3}$ is twice the warped D3-brane tension at the tip ${ }^{7}$ and $D_{\text {other }}$ represents a possible contribution from distant sources of supersymmetry breaking, e.g. in other throats.

The complete inflaton potential is then the sum of the F-term potential from moduli stabilization, plus the contribution of the antibrane,

$$
V=V_{F}\left(\rho, z_{\alpha}\right)+V_{D}(\rho, r)
$$

The canonical inflaton $\phi$ is proportional to $r$, the radial location of the D3-brane (see $\S$ B.3 for details). Using (2.21) to compute the slow-roll parameter

$$
\eta \equiv M_{P}^{2} \frac{V_{, \phi \phi}}{V}
$$

we find

$$
\eta=\frac{2}{3}+\Delta \eta(\phi)
$$

where $\Delta \eta$ arises from the dependence of the superpotential on $\phi$. If $A$ were a constant independent of $\phi$, slow-roll inflation would be impossible [6], because in that case $\eta=\frac{2}{3}$. In this paper, using the explicit result of [9] for $A(\phi)$, we will compute $\Delta \eta$ and determine whether the full potential can be flat enough for inflation. Note that the sign of $\Delta \eta$, while crucial, is not obvious a priori.

\section{Case Study: Kuperstein Embedding}

Let us consider a particularly simple and symmetric holomorphic embedding due to Kuperstein [20], which is defined by the algebraic equation

$$
f\left(z_{1}\right)=\mu-z_{1}=0
$$

where, without loss of generality, we will consider the case in which $\mu \in \mathbb{R}^{+}$. This embedding preserves an $S O(3)$ subgroup of the $S O(4)$ global symmetry acting on the $z_{i}$ coordinates of the deformed conifold. Kuperstein showed that this is a supersymmetric embedding not just for the singular conifold, but also in the full warped deformed conifold background

\footnotetext{
${ }^{7}$ A similar potential for a mobile D3-brane arises if instead of including the antibrane we generalize the throat background [32].
} 
with three-form fluxes. (For comparison, the embeddings of $[19,17]$ have so far been studied explicitly only in the $A d S_{5} \times T^{1,1}$ background). Adding just a mobile D3-brane does not break supersymmetry in the case of the non-compact throat. Therefore, the interaction between the D3-branes and D7-branes must vanish in that limit. When the throat is embedded in a compactification, the D3-brane potential can receive a contribution from the nonperturbative superpotential (2.14).

The inflaton potential $V\left(\rho, r, z_{i}\right)$ is in general a complicated function of the Kähler modulus and of the radial and angular coordinates of the D3-brane. In this section we systematically integrate out all fields except the radial coordinate, leading to an effective single-field potential for the radial inflaton.

\subsection{Multi-field potential}

\subsubsection{F-term potential}

By the results of [9], equation (3.1) implies

$$
A\left(z_{1}\right)=A_{0}\left(1-\frac{z_{1}}{\mu}\right)^{1 / n},
$$

and the F-term potential (2.13) is

$$
\begin{aligned}
V_{F}=\frac{\kappa^{2} a\left|A\left(z_{1}\right)\right|^{2} e^{-a(\rho+\bar{\rho})}}{3 U(\rho, r)^{2}} & (a(\rho+\bar{\rho})+6)+6 W_{0} \operatorname{Re}\left(\frac{e^{a \rho}}{A\left(z_{1}\right)}\right) \\
& \left.-3 \operatorname{Re}\left(\alpha_{z_{1}} z_{1}\right)+\frac{r}{a \gamma}\left(1-\frac{\left|z_{1}\right|^{2}}{2 r^{3}}\right)\left|\alpha_{z_{1}}\right|^{2}\right],
\end{aligned}
$$

where

$$
\alpha_{z_{1}} \equiv \frac{A_{z_{1}}}{A}=-\frac{1}{n\left(\mu-z_{1}\right)},
$$

and

$$
\operatorname{Re}\left(\alpha_{z_{1}} z_{1}\right)=-\frac{1}{2 n} \frac{\mu\left(z_{1}+\overline{z_{1}}\right)-2\left|z_{1}\right|^{2}}{\left|\mu-z_{1}\right|^{2}} .
$$

Note that the potential (3.3) depends only on $r, z_{1}$, and $\rho$. Therefore, it is invariant under the $S O(3)$ that acts on $z_{2}, z_{3}, z_{4}$. 


\subsubsection{Angular degrees of freedom}

\section{Imaginary part of the Kähler modulus}

First, to reduce the complexity of the multi-field potential, we integrate out the imaginary part of the Kähler modulus. Setting $\rho \equiv \sigma+i \tau$, equation (3.3) becomes

$$
\begin{aligned}
V_{F}=\frac{\kappa^{2} a|A|^{2} e^{-2 a \sigma}}{3 U^{2}}[ & (2 a \sigma+6)+6 W_{0} e^{a \sigma} \frac{\underline{\operatorname{Re}\left(\frac{e^{i a \tau}}{A}\right)}}{=} \\
& \left.-3 \operatorname{Re}\left(\alpha_{z_{1}} z_{1}\right)+\frac{r}{a \gamma}\left(1-\frac{\left|z_{1}\right|^{2}}{2 r^{3}}\right)\left|\alpha_{z_{1}}\right|^{2}\right] .
\end{aligned}
$$

We see that only the underlined term depends on $\tau$, and the potential for $\tau$ is minimized when this term is as small as possible. Because $W_{0}$ is negative, integrating out $\tau\left(z_{1}, r\right)=\operatorname{Im}(\rho)$ then amounts to the replacement

$$
\frac{e^{i a \tau}}{A} \rightarrow \frac{1}{|A|}
$$

Notice that this is not the same as setting $\tau \equiv 0$. In particular, $\tau\left(z_{1}, r\right)$ might be a complicated function, but all we need to know is (3.7).

\section{Angular directions}

The D3-brane position is described by the radial coordinate $r$ and five angles $\Psi_{i}$ on the base of the cone. The angles are periodic coordinates on a compact space, so the potential in $\Psi_{i}$ is either constant or else has discrete minima at some values $\Psi_{i}^{\star}$. We are interested in trajectories that are stable in the angular directions, so that the motion occurs purely along the radial direction.

We can therefore reduce the number of degrees of freedom by fixing the angular coordinates to the positions that minimize the potential. In Appendix $\mathrm{C}$ we show that for any Kuperstein-like embedding $f\left(z_{1}\right)=0$, these extrema in the angular directions occur only for trajectories satisfying

$$
z_{1}= \pm \frac{r^{3 / 2}}{\sqrt{2}}, \quad \Leftrightarrow \quad \frac{\partial V}{\partial \Psi_{i}}=0
$$

Furthermore, in Appendix C we examine the matrix of second derivatives, $\frac{\partial^{2} V}{\partial \Psi_{i} \partial \Psi_{j}}$, and find the conditions under which these extrema are stable minima. For the present discussion we only need one result from that section: for small $r$, the trajectory (3.8) is stable against angular fluctuations for negative $z_{1}$ and unstable for positive $z_{1}$. In Appendix B we show that the canonical inflaton field $\phi$ is well-approximated by a constant rescaling of the radial 
coordinate $r$,

$$
\phi^{2} \equiv T_{3} \hat{r}^{2}=\frac{3}{2} T_{3} r^{2}
$$

An important parameter of the brane potential is the minimal radial coordinate of the D7brane embedding $[9,20], r_{\mu}^{3} \equiv 2 \mu^{2}$, or $\phi_{\mu}^{2}=\frac{3}{2} T_{3}\left(2 \mu^{2}\right)^{2 / 3}$. The potential along the trajectory (3.8) may then be written as

$$
V(\phi, \sigma)=V_{F}(\phi, \sigma)+V_{D}(\phi, \sigma)
$$

where

$$
\begin{aligned}
V_{F}(\phi, \sigma) & =\frac{\kappa^{2} a\left|A_{0}\right|^{2}}{3} \frac{\exp (-2 a \sigma)}{U(\phi, \sigma)^{2}} g(\phi)^{2 / n}\left[2 a \sigma+6-6 \exp (a \sigma) \frac{\left|W_{0}\right|}{\left|A_{0}\right|} \frac{1}{g(\phi)^{1 / n}}\right. \\
& \left.+\frac{3}{n}\left(c \frac{\phi}{\phi_{\mu}} \pm\left(\frac{\phi}{\phi_{\mu}}\right)^{3 / 2}-\left(\frac{\phi}{\phi_{\mu}}\right)^{3}\right) \frac{1}{g(\phi)^{2}}\right] \\
V_{D}(\phi, \sigma) & =\frac{D(\phi)}{U(\phi, \sigma)^{2}},
\end{aligned}
$$

and

$$
g(\phi) \equiv\left|1 \mp\left(\frac{\phi}{\phi_{\mu}}\right)^{3 / 2}\right|, \quad U(\phi, \sigma) \equiv 2 \sigma-\frac{\sigma_{0}}{3} \frac{\phi^{2}}{M_{P}^{2}} .
$$

Here we have introduced the constant

$$
c \equiv \frac{1}{4 \pi \gamma r_{\mu}^{2}}=\frac{9}{4 n a \sigma_{0} \frac{\phi_{\mu}^{2}}{M_{P}^{2}}} .
$$

This two-field potential is the input for our numerical study in $§ 4.3$.

\subsection{Effective single-field potential}

\section{Real part of the Kähler modulus}

Having reduced the potential to a function of two real fields, $\phi$ and $\sigma$, we integrate out $\sigma$ by assuming $^{8}$ that it evolves adiabatically while remaining in its instantaneous minimum $\sigma_{\star}(\phi)$, which is defined implicitly by

$$
\left.\partial_{\sigma} V\right|_{\sigma_{\star}(\phi)}=0 .
$$

\footnotetext{
${ }^{8} \mathrm{We}$ are assuming that $\sigma$ is much more massive than $\phi$. This may not be valid for a truly generic configuration of a D3-brane in a compact space, but we are specifically interested in cases in which the potential for $\phi$ has been fine-tuned to be flat. Thus, when slow-roll inflation is possible at all, the adiabatic approximation is justified. See also [34].
} 
This leads to the effective single-field potential

$$
\mathbb{V}(\phi) \equiv V\left(\sigma_{\star}(\phi), \phi\right)
$$

In general, we are not able to solve equation (3.15) analytically for $\sigma_{\star}(\phi)$, so we perform this final step numerically $(\S 4.3)$. Nevertheless, in Appendix D we derive useful approximate analytical solutions for the stabilized volume modulus and its dependence on $\phi$ (similar results were derived independently in [33]). Here, we cite the basic results of that section. First, the critical value $\sigma_{F}$ of the Kähler modulus before uplifting is determined by $\left.D_{\rho} W\right|_{\phi=0, \sigma_{F}}=0$, or equivalently [16],

$$
3 \frac{\left|W_{0}\right|}{\left|A_{0}\right|} e^{a \sigma_{F}}=2 a \sigma_{F}+\left.3 \quad \Rightarrow \quad \frac{\partial V_{F}}{\partial \sigma}\right|_{\sigma_{F}}=0 .
$$

We now show how the Kähler modulus is shifted away from $\sigma_{F}$ by the inclusion of a braneantibrane pair.

\section{Shift induced by the uplifting}

Adding an anti-D3-brane to lift the KKLT AdS minimum to a dS minimum induces a small shift in the stabilized volume, $\sigma_{F} \rightarrow \sigma_{F}+\delta \sigma \equiv \sigma_{\star}(0) \equiv \sigma_{0}$, where

$$
\delta \sigma \approx \frac{s}{a^{2} \sigma_{F}} \ll 1 \ll \sigma_{F} .
$$

Here we found it convenient to define the ratio of the antibrane energy to the F-term energy before uplifting, i.e. when $\sigma=\sigma_{F}$,

$$
s \equiv \frac{\left(D+D_{\text {other }}\right) U^{-2}\left(0, \sigma_{F}\right)}{\left|V_{F}\left(0, \sigma_{F}\right)\right|},
$$

where stability of the volume modulus in a metastable de Sitter vacuum requires $1<$ $s \lesssim \mathcal{O}(3)$. Although $\delta \sigma$ is small, it appears in an exponent in (3.11), so that its effect there has to be considered,

$$
3 \frac{\left|W_{0}\right|}{\left|A_{0}\right|} e^{a \sigma_{0}} \approx 2 a \sigma_{0}+3+2 s .
$$

When the D3-brane is near the tip, $\phi \approx 0$, the Kähler modulus remains at $\sigma_{0}$. Using this constant value even when the brane is at finite $\phi$ suffices for understanding the basic qualitative features of the potential (3.11). However, important quantitative details of the potential depend sensitively on the dependence of $\sigma_{\star}$ on $\phi$; see Appendix $\mathrm{D}$ and Ref. [34]. 


\section{Shift induced by D3-brane motion}

In Appendix D we derive the following analytic approximation to the dependence of the stabilized volume on the D3-brane position:

$$
\sigma_{\star}(\phi) \approx \sigma_{0}\left[1+c_{3 / 2}\left(\frac{\phi}{\phi_{\mu}}\right)^{3 / 2}\right]
$$

where

$$
c_{3 / 2} \approx \frac{1}{n} \frac{1}{a \sigma_{F}}\left[1-\frac{1}{2 a \sigma_{F}}\right]
$$

This expression is valid along $z_{1}=-\frac{r^{3 / 2}}{\sqrt{2}}$, which we argue below is the interesting case in which the potential is stable in the angular directions.

Analytic single-field potential

Along the trajectory $z_{1}=-\frac{r^{3 / 2}}{\sqrt{2}}$, the inflaton potential is

$$
\begin{gathered}
\mathbb{V}(\phi)=\frac{\kappa^{2} a\left|A_{0}\right|^{2}}{3} \frac{\exp \left(-2 a \sigma_{\star}(\phi)\right)}{U\left(\phi, \sigma_{\star}(\phi)\right)^{2}} g(\phi)^{2 / n}\left[2 a \sigma_{\star}(\phi)+6-6 \exp \left(a \sigma_{\star}(\phi)\right) \frac{\left|W_{0}\right|}{\left|A_{0}\right|} \frac{1}{g(\phi)^{1 / n}}\right. \\
\left.+\frac{3 c}{n} \frac{\phi}{\phi_{\mu}} \frac{1}{g(\phi)^{2}}-\frac{3}{n}\left(\frac{\phi}{\phi_{\mu}}\right)^{3 / 2} \frac{1}{g(\phi)}\right]+\frac{D(\phi)}{U\left(\phi, \sigma_{\star}(\phi)\right)^{2}},
\end{gathered}
$$

where $\sigma_{\star}(\phi)$ can be determined numerically or approximated analytically by (3.21). Using the analytic result (3.21) in (3.23) captures the basic qualitative features of the potential, but is insufficient to assess detailed quantitative questions. In particular, by using (3.21) one systematically underestimates the total number of $e$-folds supported by the potential (see Appendix D and Ref. [34]).

The inflaton potential (3.23) is one of our main results.

\section{Search for an Inflationary Trajectory}

In the preceding section, we derived the inflaton potential (3.23) along the angularly-stable trajectory $z_{1}=-\frac{r^{3 / 2}}{\sqrt{2}}$. We will now explore this potential and establish that slow roll inflation is possible for a certain range of parameters. First, in $\S 4.1$, we present a few analytic results about the curvature of the potential. Then, in $\S 4.2$, we describe the constraints on the parameters of the model that are dictated by the structure of the compactification. Finally, in $\S 4.3$, we present the results of a numerical study of the potential. 


\subsection{Analytic considerations}

Let us briefly recall the reason for computing the effect of $A(\phi)$ on the inflaton potential. Kachru et al. [6] derived $\eta=\frac{2}{3}$ for the case $A=$ const., and suggested that the inflatondependence of the nonperturbative superpotential, $A(\phi)$, could contribute corrections to the inflaton mass, which, if of the right sign, could accidentally make $\eta$ small. This reasonable expectation hinges on the presence of quadratic corrections to the inflaton potential.

We now argue that in view of the result (2.15), wrapped D7-branes give no purely quadratic corrections to the inflaton potential. To see this, we note that the holomorphic coordinates on the conifold scale as fractional powers of $\phi,\left|z_{i}\right| \propto \phi^{3 / 2}$, and $A\left(z_{i}\right)$ is a holomorphic function of the $z_{i}$ coordinates [9]. This observation implies that the inflaton potential is of the form

$$
\frac{\mathbb{V}(\phi)}{\mathbb{V}(0)}=1+\frac{1}{3} \frac{\phi^{2}}{M_{P}^{2}}+v(\phi),
$$

where $v(\phi)$ contains no quadratic terms. The slow roll parameter $\eta$, which needs to be very small for sustained slow roll inflation, is

$$
\eta \equiv M_{P}^{2} \frac{\mathbb{V}, \phi \phi}{\mathbb{V}}=\frac{2}{3}+M_{P}^{2} v_{, \phi \phi}
$$

Because $v_{, \phi \phi}$ contains no constant term, there is no possibility of cancelling the $\frac{2}{3}$ uniformly, for the entire range of $\phi$. Instead, we can at best hope to cancel the $\frac{2}{3}$ at some special point(s) $\phi_{0}$ obeying $v_{, \phi \phi}\left(\phi_{0}\right)=-\frac{2}{3}$.

Using the explicit form (3.23) and expanding for small $\phi / \phi_{\mu}$, we find

$$
\eta=\frac{2}{3}-\eta_{-1 / 2}\left(\frac{\phi}{\phi_{\mu}}\right)^{-1 / 2}+\cdots
$$

where

$$
\eta_{-1 / 2} \approx \frac{M_{P}^{2}}{\phi_{\mu}^{2}} \frac{3(4 s-3)}{8 n(s-1)} \frac{1}{a \sigma_{0}}>0 .
$$

Hence, $\eta<0$ sufficiently close to the tip. On the other hand, we find that for $\phi \gg \phi_{\mu}, \eta>0$. By continuity, $\eta$ must vanish at some intermediate location $\phi_{0}$.

The precise location of $\phi_{0}$ is a parameter-dependent question. For this purpose, the most important parameter is the minimal radius $\phi_{\mu}$ of the D7-branes. Notice that (4.2) can be written as

$$
\eta=\frac{2}{3}+\frac{M_{P}^{2}}{\phi_{\mu}^{2}} v_{, x x}
$$

where $x \equiv \frac{\phi}{\phi_{\mu}}$ and $v_{x x}$ is insensitive to $\phi_{\mu}$ (see (3.23)). From (4.3) we see that the second term in (4.5) dominates near the tip, giving a large negative $\eta$. This implies the opportunity 
for a small $\eta$ by cancellation against the positive $\frac{2}{3}$. However, only if $\phi_{\mu}$ is not too small can this cancellation be achieved inside the throat. Otherwise, $\eta$ remains negative throughout the regime of interest. We conclude that for small $\phi_{\mu}, \phi_{0}$ is outside the throat, and hence outside the validity of our construction.

\subsection{Parameters and microscopic constraints}

Let us describe the microscopic parameters that determine the inflaton potential (3.23). In view of (3.19), the D-term $D_{\text {other }}+2 T_{3} h_{0}^{-1}$ and $W_{0}$ are represented by $s$ and by $\omega_{F}=$ $a \sigma_{F}$, respectively. Next, the prefactor $A_{0}$ only appears as an overall constant rescaling the height of the potential, so we set $A_{0} \equiv 1$. The shape of the inflaton potential is therefore determined by $n, \omega_{F}, s$ and $\phi_{\mu}$. As we now explain, microscopic constraints lead to important restrictions on the allowed parameter ranges and induce non-trivial correlations among the above parameters.

First, the range of the radial coordinate $r$ affects the four-dimensional Planck mass, because a longer throat makes a larger contribution to the volume of the compact space. In [21] two of us (D.B. and L.M.) showed that this creates a strong constraint on the allowed field range of the inflaton field $\phi$ (see also Appendix B)

$$
\frac{\Delta \phi}{M_{P}}<\frac{2}{\sqrt{N}}
$$

Here we use the field range bound (4.6) to constrain the microscopically viable range of $\phi_{\mu}$, the minimal radial extent of the D7-branes in canonical units. For this purpose we find it convenient to write the bound in the form

$$
\frac{\phi_{\mu}^{2}}{M_{P}^{2}}=\frac{1}{Q_{\mu}^{2}} \frac{1}{B_{6}} \frac{4}{N}
$$

where $B_{6} \equiv \frac{V_{6}^{w}}{\left(V_{6}^{w}\right)_{\text {throat }}}>1$ parameterizes the relative contribution of the throat to the total (warped) volume of the compact space, and

$$
Q_{\mu} \equiv \frac{r_{\mathrm{UV}}}{r_{\mu}}
$$

is a measure of how far into the throat the four-cycle extends. Applicability of the results of [9] requires $Q_{\mu}=\frac{\phi_{\mathrm{UV}}}{\phi_{\mu}}>1$.

Second, the warped volume $V_{\Sigma_{4}}^{w}$ of the wrapped four-cycle $\Sigma_{4}$ is bounded below by the warped volume in the throat region,

$$
V_{\Sigma_{4}}^{w}=\left(V_{\Sigma_{4}}^{w}\right)_{\text {throat }}+\left(V_{\Sigma_{4}}^{w}\right)_{\text {bulk }} \geq\left(V_{\Sigma_{4}}^{w}\right)_{\text {throat }}
$$


In Appendix B.2 we compute $\left(V_{\Sigma_{4}}^{w}\right)_{\text {throat }}$ for the Kuperstein embedding

$$
T_{3}\left(V_{\Sigma_{4}}^{w}\right)_{\text {throat }}=\frac{3}{2} N \log Q_{\mu} .
$$

In $\S 2$ we explained how the unperturbed warped four-cycle volume relates to the Kähler modulus of the compactification (more details can be found in Refs. [9, 12] and Appendix B). If we use $B_{4} \equiv \frac{V_{\Sigma_{4}}^{w}}{\left(V_{\Sigma_{4}}^{w}\right) \text { throat }}>1$ to parameterize the relative contribution of the throat to the total warped volume of the four-cycle wrapped by the D7-branes, then we can relate the vev of the Kähler modulus to microscopic parameters of the compactification

$$
\omega_{F} \approx \omega_{0} \approx \frac{3}{2} \frac{N}{n} B_{4} \log Q_{\mu} .
$$

We require $\omega_{0}<\mathcal{O}(30)$, because otherwise the inflation scale will be too low - see Appendix B.

The constraints (4.7), (4.11) will play an essential role in our analysis. We will find that inflationary configurations are rather easy to find if these constraints are neglected, but imposing them dramatically decreases the parameter space suitable for inflation.

\subsubsection{Bulk contributions to the volume}

We have just introduced two parameters, $B_{4}=\frac{V_{\Sigma_{4}}^{w}}{\left(V_{\Sigma_{4}}^{w}\right) \text { throat }}$ and $B_{6}=\frac{V_{6}^{w}}{\left(V_{6}^{w}\right)_{\text {throat }}}$, that represent ratios of total volumes to throat volumes. In the throat, we have access to an explicit CalabiYau metric and can compute the volumes directly. This metric data in the throat is one of the main reasons that warped D-brane inflation can be studied explicitly. In contrast, we have very little data about the bulk, so we cannot compute $B_{4}$ and $B_{6}$.

Fortunately, these parameters do not directly enter the potential. Instead, they appear in the compactification constraints (4.7) and (4.11), and thereby affect the microscopically allowable ranges of the other parameters, such as $\phi_{\mu}$ and $Q_{\mu}$. In particular, when $B_{6}$ is large, the range of $\phi_{\mu}$ is reduced, because the throat is shorter in four-dimensional Planck units. In the numerical investigation of $\S 4.3$, we find that inflation is possible inside the throat, and our construction is self-consistent, provided that $B_{4} / B_{6} \gtrsim 2$. For concreteness, we take $B_{4} \sim 9, B_{6} \sim 1.5$ in the remainder. This means that the throat contributes a greater share of the total six-volume than it does of the wrapped four-cycle volume: in other words, the wrapped four-cycle only enters the upper reaches of the throat. Although we expect that such a configuration can be realized, it will be valuable to find a fully explicit construction. We note, however, that a very large value of $B_{4}$, implying that the D7-brane is hardly in the throat at all, would mean that the result of $[9]$ is inapplicable, because the correction to the four-cycle volume is then dominated by the correction to the uncomputable bulk portion of the volume. 


\subsubsection{Parameter choices}

Although a systematic study of the full multi-dimensional parameter space would undoubtedly be instructive, we here employ a simpler and more transparent strategy that we believe nevertheless accurately portrays the range of possibilities. To this end, we set some of the discrete parameters to reasonable values and then scan over the remaining parameters. Let us emphasize that although the precise values chosen here are not important, it is important that we were able to find regions in parameter space where all our approximations are valid and all the compactification constraints are satisfied.

First, we fix $n \rightarrow 8$. This helps to reduce the degree to which the volume shifts during inflation, as from (3.22), $c_{3 / 2} \propto n^{-1}$. Numerical study of the case $n=2$ yields results qualitatively similar to those we present here, but the analytical treatment is more challenging. To ignore backreaction of the wrapped branes on the background geometry, we require that the background D3-brane charge exceeds the number of wrapped branes, $\frac{N}{n}>1$. For concreteness, we use $N=32$. Finally, as previously stated, we take $B_{4} \sim 5, B_{6} \sim 2$.

This allows us to impose the microscopic constraints (4.7), (4.11) on the compactification volume $\omega_{F}$ and the wrapped brane location $\phi_{\mu}$ in terms of a single parameter $Q_{\mu}$. The remaining parameters that determine the potential are then $Q_{\mu}$ (4.8) and $s$ (3.19).

To search for inflationary solutions, we scanned over $Q_{\mu}$ and $s$, treating both as continuous parameters, although they are in principle determined by discrete flux input. Here, for simplicity of presentation, we will fix $Q_{\mu}$ to a convenient value, $Q_{\mu}=1.2$, and only exhibit the scanning over $s$. To interpret this scanning in microphysical terms, we recall that, for fixed F-term potential and for fixed supersymmetry breaking (corresponding to the parameter $D_{\text {other }}$ ) outside the throat, $s$ is determined by $D=2 T_{3} h_{0}^{-1}$, where $h_{0}$ is given in (2.7) and is

of order $h_{0} \approx \exp \left(\frac{8 \pi K}{3 g_{s} M}\right)$. The values of $h_{0}$ we will consider can be achieved for reasonable values of $K, M, g_{s}$.

In summary, we have arranged that all consistency conditions are satisfied, and all parameters except for the amount of uplifting, $s$, are fixed. As we vary the uplifting, the shape of the potential (3.23) will change. As we shall now see, for a certain range of values of $s$ the potential becomes flat enough for prolonged inflation.

\subsection{Numerical results}

The first observation we make about (3.23) is that, near the parameter values we have indicated, it is generically non-monotonic. In fact, the potential has a metastable minimum ${ }^{9}$

\footnotetext{
${ }^{9}$ A D3-brane located in this metastable minimum contributes to the breaking of supersymmetry. It would be extremely interesting to use a configuration of a D3-brane and a moduli-stabilizing D7-brane stack to uplift to a de Sitter vacuum. Here we have not quite accomplished this: we have, of course, included an
} 


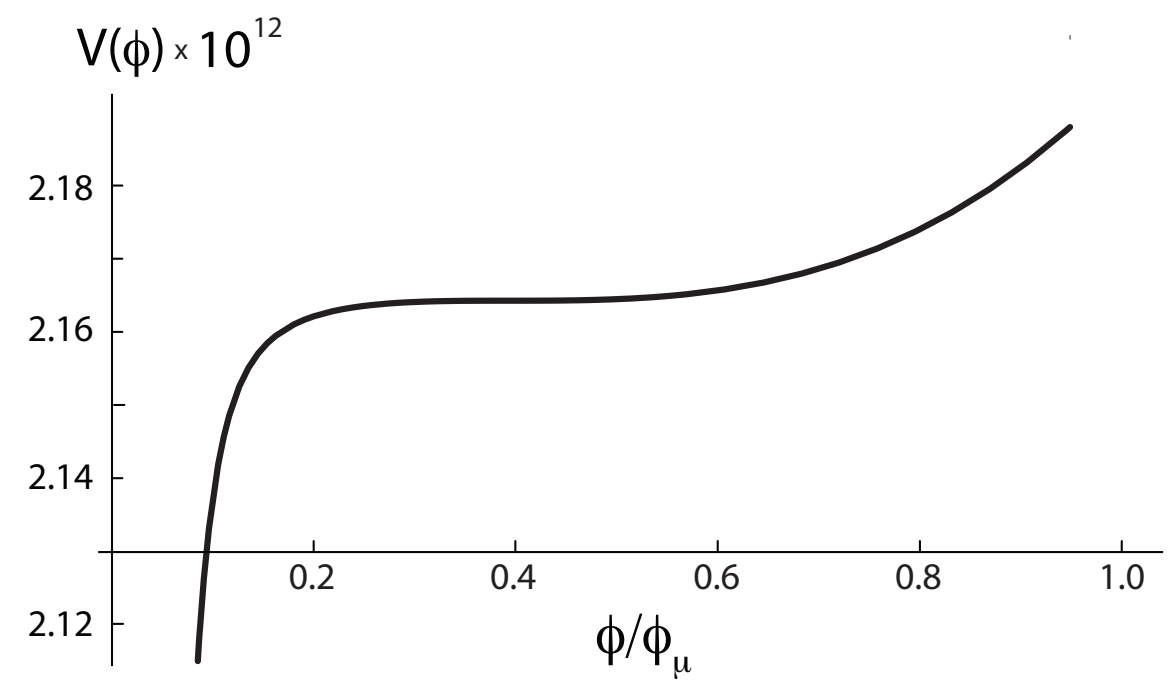

Figure 2: Inflaton potential $\mathbb{V}(\phi)$.

Compactification data: $n=8, \omega_{F}=10, N=32, Q_{\mu}=1.2, B_{6}=1.5, B_{4}=9, s=1.1$, which implies $\phi_{\mu}=0.25, W_{0}=-3.432 \times 10^{-4}, D+D_{\text {other }}=1.2 \times 10^{-8}, \omega_{0} \approx 10.1$.

at some distance from the tip. We are confident that this is a minimum and not a saddle point, because we have explicitly shown in the Appendices that the curvature of the potential in the angular directions is non-negative. (The curvature is zero along directions protected by the unbroken $S O(3)$ symmetry of the background, and positive in the other directions.) Moreover, we have shown that the potential is stable with respect to changes in the Kähler modulus.

Next, we notice that as we vary $s$, the metastable minimum grows more shallow, and the two zeroes of $V^{\prime}$, the local maximum and the local minimum, approach each other. A zero of $V^{\prime \prime}$ is trapped in the shrinking range between these two zeroes of $V^{\prime}$. For a critical value of $s$, the zero of $V^{\prime \prime}$ and the two zeroes of $V^{\prime}$ coincide, and the potential has an inflection point. As $s$ changes further, the potential becomes strictly monotonic.

We therefore find that there exists a range of $s$ for which both the first and second derivatives of the potential approximately vanish. This is an approximate inflection point. In the next section we discuss a phenomenological model that captures the essential features of (3.23) in the vicinity of this inflection point.

anti-D3-brane as well, which is well-known to accomplish the uplifting by itself [16]. If this antibrane is removed, the structure of the potential changes, and it is not clear from our results so far that a remaining D3-brane would suffice to uplift to a de Sitter vacuum. We leave this as a promising direction for future work. 

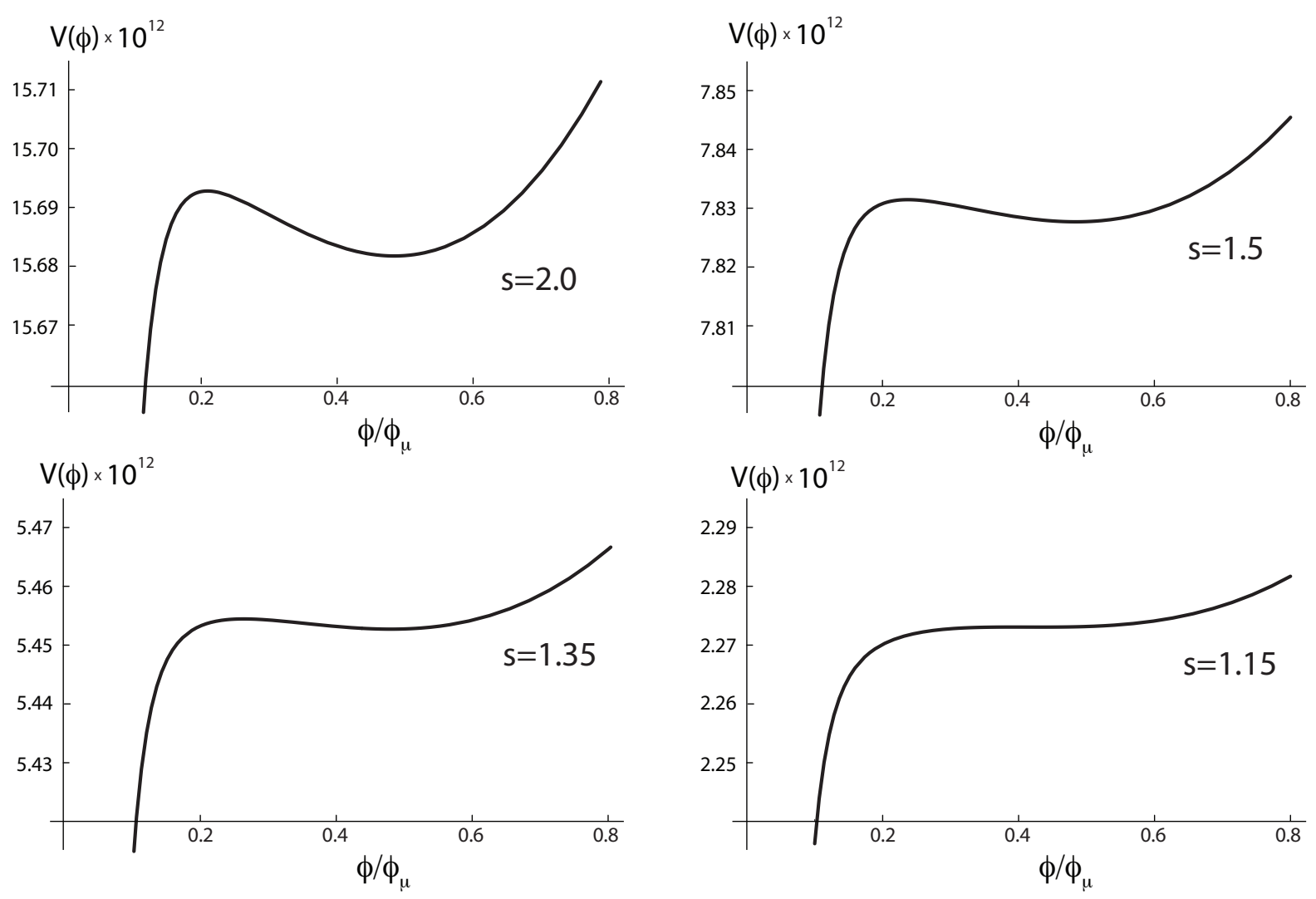

Figure 3: The inflaton potential $\mathbb{V}(\phi)$ as a function of $s$.

The transition from metastability to monotonicity is shown; old inflation and new inflation are continuously connected.

\subsection{Phenomenological model: cubic inflation}

We have shown that inflationary solutions in the Kuperstein embedding arise near an inflection point at $\phi=\phi_{0}$, where the potential is very well approximated by the cubic form $[35]^{10}$

$$
\mathbb{V}=V_{0}+\lambda_{1}\left(\phi-\phi_{0}\right)+\frac{1}{3 !} \lambda_{3}\left(\phi-\phi_{0}\right)^{3}
$$

\footnotetext{
${ }^{10}$ Ref. [36] has developed an inflation model within the minimal supersymmetric standard model (MSSM) that has a similar cubic phenomenology. We thank Justin Khoury for bringing this model to our attention. Inflection point inflation in the context of string theory, with the inflaton corresponding to the compactification volume, has been considered in [37].
} 
Prolonged inflation requires smallness of the slow roll parameters, $\epsilon, \eta \ll 1$. From (4.12) we find ${ }^{11}$

$$
\begin{aligned}
\epsilon & \equiv \frac{1}{2}\left(\frac{\mathbb{V}, \phi}{\mathbb{V}}\right)^{2} \approx \frac{1}{2}\left(\frac{\lambda_{1}+\frac{1}{2} \lambda_{3}\left(\phi-\phi_{0}\right)^{2}}{V_{0}}\right)^{2}, \\
\eta & \equiv \frac{\mathbb{V}_{, \phi \phi}}{\mathbb{V}} \approx \frac{\lambda_{3}}{V_{0}}\left(\phi-\phi_{0}\right) .
\end{aligned}
$$

The number of $e$-folds between some value $\phi$ and the end of inflation $\phi_{\text {end }}$ is then

$$
N_{e}(\phi)=\int_{\phi_{\text {end }}}^{\phi} \frac{\mathrm{d} \phi}{\sqrt{2 \epsilon}}=\left.\frac{N_{\text {tot }}}{\pi} \arctan \left(\frac{\eta(\phi)}{2 \pi N_{\text {tot }}^{-1}}\right)\right|_{\phi_{\text {end }}} ^{\phi},
$$

where

$$
N_{\text {tot }} \equiv \int_{-\infty}^{\infty} \frac{\mathrm{d} \phi}{\sqrt{2 \epsilon}}=\pi \sqrt{\frac{2 V_{0}^{2}}{\lambda_{1} \lambda_{3}}}
$$

In (4.13) and (4.14) we have set $\mathbb{V}(\phi) \approx V_{0}$ in the denominators, while in (4.16) we extended the integral from the range where $|\eta|<1$ to infinity. These approximations are very good in the regime

$$
\frac{V_{0}}{\lambda_{3}} \ll 1, \quad \frac{V_{0}}{\sqrt{\lambda_{1} \lambda_{3}}} \gg 1 .
$$

The first of these conditions guarantees that inflation is of the small-field type, while the second implies that $N_{\text {tot }} \gg 1$. We will be interested in $N_{\text {tot }} \geq N_{\mathrm{CMB}} \sim 60$.

Equation (4.15) shows that there are $\frac{1}{2} N_{\text {tot }} e$-folds during which $|\eta|<2 \pi N_{\text {tot }}^{-1}$; see Figure 4. For large $N_{\text {tot }}$ this implies that there is a large range of $e$-folds where $\eta$ is small and the scalar perturbation spectrum is nearly scale-invariant. Predicting the scalar spectral index in these models is non-trivial:

$$
n_{s}-1=\left.(2 \eta-6 \epsilon)\right|_{\phi_{\mathrm{CMB}}} \approx 2 \eta\left(\phi_{\mathrm{CMB}}\right) .
$$

The scalar spectral index on CMB scales can be red, blue or even perfectly scale-invariant depending on where $\phi_{\mathrm{CMB}}$ is relative to the inflection point. If inflation only lasts for the minimal number of $e$-folds to solve the horizon and flatness problems then the scalar spectrum is blue. If the potential is flatter than this, so that $\epsilon$ is smaller, inflation lasts longer and $\phi_{\mathrm{CMB}}$ is more likely to be smaller than $\phi_{0}$. The spectrum is then red, since $\eta\left(\phi_{\mathrm{CMB}}<\phi_{0}\right)<0$.

More concretely, we can evaluate $n_{s}$ by inverting (4.15) at $\phi_{\mathrm{CMB}}$ where $N_{e}\left(\phi_{\mathrm{CMB}}\right) \equiv$ $N_{\mathrm{CMB}} \sim 60$, and using $\eta\left(\phi_{\text {end }}\right)=-1$. This gives

$$
n_{s}-1=\frac{4 \pi}{N_{\mathrm{tot}}} \tan \left(\pi \frac{N_{\mathrm{CMB}}}{N_{\mathrm{tot}}}-\arctan \left(\frac{N_{\mathrm{tot}}}{2 \pi}\right)\right) .
$$

\footnotetext{
${ }^{11}$ In this section we set $M_{P} \equiv 1$.
} 


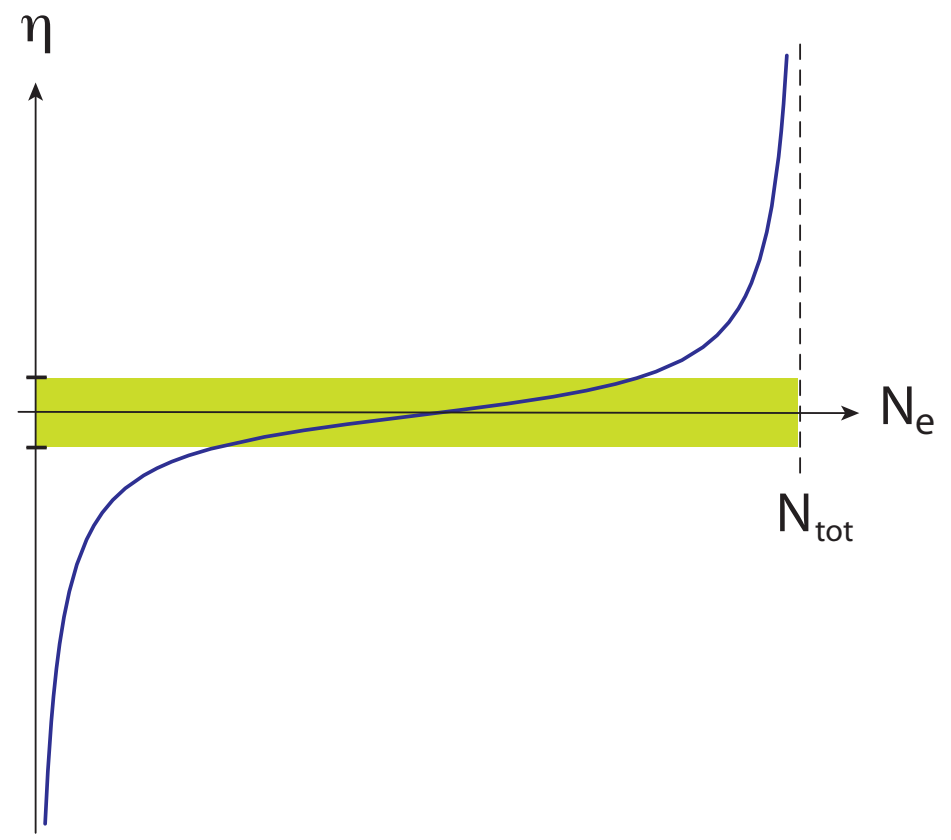

Figure 4: $\eta(\phi)$ as a function of the number of $e$-folds of inflation, $N_{e}$. In the green band, $|\eta|<2 \pi N_{\text {tot }}^{-1}$.

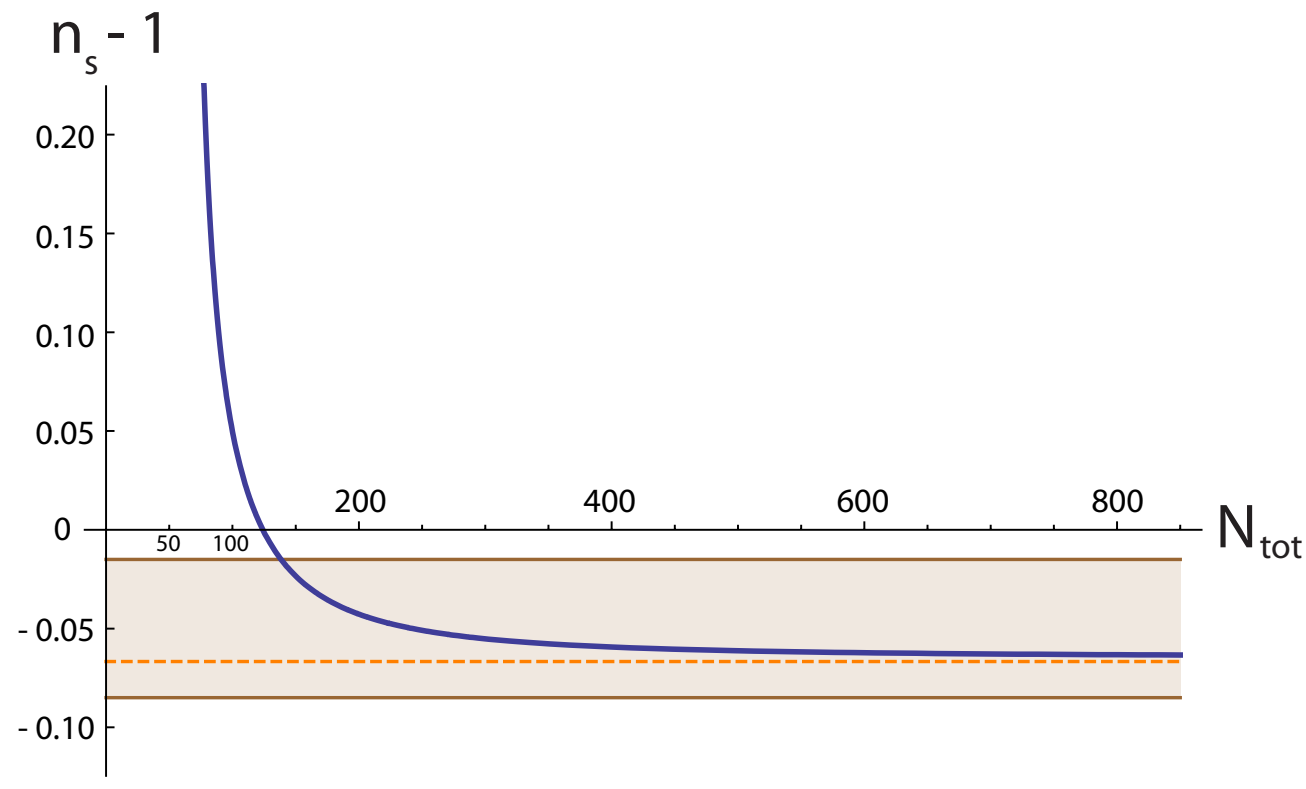

Figure 5: Spectral index $n_{s}$, evaluated on CMB scales, as a function of the total number of $e$-folds of inflation, $N_{\text {tot }}$.

The light band gives the WMAP3 $2 \sigma$ limit on $n_{s}$ (for $r \equiv 0$ ) [3]. 
Using $\arctan x=\frac{\pi}{2}-x^{-1}+\mathcal{O}\left(x^{-3}\right)$ we furthermore find

$$
n_{s}-1=\frac{4 \pi}{N_{\mathrm{tot}}} \tan \left(\pi \frac{N_{\mathrm{CMB}}+2}{N_{\mathrm{tot}}}-\frac{\pi}{2}+\mathcal{O}\left(N_{\mathrm{tot}}^{-3}\right)\right) .
$$

Using $N_{\mathrm{CMB}}+2 \approx N_{\mathrm{CMB}} \gg 1$, we may simplify this to

$$
n_{s}-1 \approx-\frac{4 \pi}{N_{\mathrm{tot}}} \cot \left(\pi \frac{N_{\mathrm{CMB}}}{N_{\mathrm{tot}}}\right)
$$

which has the expansion

$$
n_{s}-1 \approx-\frac{4}{N_{\mathrm{CMB}}}+\frac{4 \pi^{2}}{3} \frac{N_{\mathrm{CMB}}}{N_{\mathrm{tot}}^{2}}+\mathcal{O}\left(\frac{N_{\mathrm{CMB}}^{3}}{N_{\mathrm{tot}}^{4}}\right) .
$$

Equation (4.21) is plotted in Figure 5. We note the following properties of this result. For $N_{\text {tot }}$ not much greater than $N_{\mathrm{CMB}}$ the spectrum is strongly blue and the model is hence ruled out by recent observations [3] (in this regime the slow-roll formulae we have used are not good approximations, but a more exact treatment gives similar results). For $N_{\text {tot }} \approx 2 N_{\mathrm{CMB}}$ the spectrum on CMB scales is exactly scale-invariant. For $N_{\text {tot }} \gtrsim 2 N_{\mathrm{CMB}}$ the spectrum is red and asymptotes to the lower limit $n_{s} \rightarrow 1-4 / N_{\mathrm{CMB}} \approx 0.93$ for $N_{\text {tot }} \gg N_{\mathrm{CMB}}$. This asymptotic limit, which has been noted in studies of inflation near an inflection point [36, 37], is more strongly red than is typical in single-field inflation models.

Given the explicit expression (4.21) for the spectral index $n_{s}\left(N_{e}\right)$ we can compute its scale-dependence or 'running':

$$
\begin{aligned}
\alpha_{s} \equiv \frac{d n_{s}}{d \ln k}=-\left.\frac{d n_{s}}{d N_{e}}\right|_{N_{e}=N_{\mathrm{CMB}}} & =-\frac{4 \pi^{2}}{N_{\mathrm{tot}}^{2}} \sin ^{-2}\left(\pi \frac{N_{\mathrm{CMB}}}{N_{\mathrm{tot}}}\right) \\
& \approx-\frac{4}{N_{\mathrm{CMB}}^{2}}-\frac{4 \pi^{2}}{3} \frac{1}{N_{\mathrm{tot}}^{2}}+\mathcal{O}\left(\frac{N_{\mathrm{CMB}}^{2}}{N_{\mathrm{tot}}^{4}}\right) .
\end{aligned}
$$

The running can be large for models with blue spectral tilt $\left(N_{\text {tot }} \sim N_{\mathrm{CMB}}\right)$, but is small for models with red spectra. The asymptotic value for $N_{\text {tot }} \gg N_{\mathrm{CMB}}$ is $\alpha_{s} \rightarrow-4 / N_{\mathrm{CMB}}^{2} \approx$ $-10^{-3}$. Notice that because both the tilt $n_{s}-1$ and the running $\alpha_{s}$ are determined by $N_{\text {tot }}$ alone (for fixed $N_{\mathrm{CMB}}$ ), this phenomenological model is predictive.

It is possible to arrange for the magnitude of scalar perturbations on CMB scales to be small,

$$
\Delta_{\mathcal{R}}^{2}=\left.\frac{1}{24 \pi^{2}} \frac{\mathbb{V}}{\epsilon}\right|_{\phi_{\mathrm{CMB}}} \approx \frac{1}{12 \pi^{2}} \frac{V_{0}^{3}}{\lambda_{1}^{2}} \approx 2.4 \times 10^{-9},
$$

by adjusting the overall scale of inflation.

Let us comment briefly on some general difficulties in inflection point inflation. Since inflation is restricted to a small region around the inflection point, an immediate concern 
is the question of initial conditions. In particular, how sensitive is the present scenario to the initial position and velocity of the D3-brane? What fraction of initial conditions lead to overshoot rather than to inflation? A complete analysis of these questions is beyond the scope of the present paper; see [33, 37] for a discussion of some aspects of this problem.

Since this is a small-field model, it is sensitive to small corrections in the slope of the potential (see §6). These corrections are important for both the background evolution, i.e. the number of $e$-folds of inflation, and for the perturbation spectrum.

Finally, we note that the appearance of the inflection point feature depends sensitively on the use of the adiabatic approximation for integrating out the volume modulus. One might therefore be worried about cases in which the exact two-field evolution is not well captured by this approximation and a more detailed numerical study of the two-field evolution is required. ${ }^{12}$

\section{Comments on Other Embeddings}

The previous two sections contained a detailed discussion of the D3-brane potential for the Kuperstein embedding. We derived important microscopic constraints, analyzed the fine-tuning problem involved in realizing inflationary solutions, and studied the resulting cosmological dynamics.

In this section we will make some brief remarks about other embeddings. When applicable we will emphasize the differences and similarities to the Kuperstein case. This will illustrate the special status of the Kuperstein embedding.

First, we give a simple proof that for an infinite class of D7-brane embeddings, the ACR embeddings, there always exist trajectories for which no amount of fine-tuning can flatten the inflaton potential. Areán, Crooks and Ramallo (ACR) [17] studied supersymmetric four-cycles in the conifold described by the embedding equations

$$
f\left(w_{i}\right)=\mu^{P}-\prod_{i=1}^{4} w_{i}^{p_{i}}=0,
$$

where $p_{i} \in \mathbb{Z}, P \equiv \sum_{i=1}^{4} p_{i}$ and $\mu^{P} \in \mathbb{C}$ are constants defining the embedding. Here $w_{i} \in \mathbb{C}$ are alternative coordinates on the conifold that follow from the $z_{i}$ coordinates by a linear transformation (see Appendix A). The conifold constraint in these coordinates is $w_{1} w_{2}-w_{3} w_{4}=0$. By requiring that the $p_{i}$ are non-negative we can restrict attention to

\footnotetext{
${ }^{12}$ This important problem has been explored in the subsequent work [34] where the validity of the adiabatic approximation is explicitly confirmed.
} 
four-cycles that do not reach the tip of the conifold. Two simple special cases of the ACR embeddings (5.1) are the Ouyang embedding [19], $w_{1}=\mu$, and the Karch-Katz embedding [38], $w_{1} w_{2}=\mu^{2}$.

To study the ACR embeddings in a unified way we define a collective coordinate $\Phi$

$$
\Phi^{P} \equiv \prod_{i=1}^{4} w_{i}^{p_{i}}
$$

such that

$$
A\left(\Phi^{P}\right)=A_{0}\left(1-\frac{\Phi^{P}}{\mu^{P}}\right)^{1 / n}
$$

and

$$
\sum_{i} w_{i} \alpha_{w_{i}}=\sum_{i} p_{i} \Phi^{P} \alpha_{\Phi^{P}}=P \Phi^{P} \alpha_{\Phi^{P}}
$$

where

$$
\alpha_{\Phi^{P}} \equiv \frac{1}{A} \frac{\partial A}{\partial \Phi^{P}}=-\frac{1}{n} \frac{1}{\mu^{P}-\Phi^{P}} .
$$

Next, we consider the part of the F-term potential that depends on derivatives of the superpotential with respect to the brane coordinates

$$
\Delta V_{F}=-\frac{\kappa^{2} a|A|^{2} e^{-2 a \sigma}}{U^{2}}\left[3 \operatorname{Re}\left(w^{i} \alpha_{w_{i}}\right)-\frac{1}{a \gamma} \hat{k}_{w}^{i \bar{\gamma}} \alpha_{w_{i}} \alpha_{\overline{w_{j}}}\right],
$$

where

$$
\operatorname{Re}\left(w^{i} \alpha_{w_{i}}\right)=P \operatorname{Re}\left(\Phi^{P} \alpha_{\Phi^{P}}\right)
$$

and

$$
\frac{1}{\gamma} \hat{k}_{w}^{i \bar{\gamma}} \alpha_{w_{i}} \alpha_{\overline{w_{j}}}=\frac{1}{\gamma r^{2}}\left|\alpha_{\Phi^{P}}\right|^{2}\left|\Phi^{P}\right|^{2} \times\left\{\frac{1}{2} P^{2}+\left(p_{1}-p_{2}\right)^{2}+\left(p_{3}-p_{4}\right)^{4}+Z\left(w_{i}\right)\right\} .
$$

Here, we found it convenient to write the Kähler metric $\hat{k}_{w}^{i \bar{\jmath}}$ in $S O(4)$-invariant form (see Appendix A.2) and defined the function

$$
\begin{aligned}
Z \equiv & +\left|p_{1} \frac{w_{4}}{w_{1}}+p_{3} \frac{w_{2}}{w_{3}}\right|^{2}+\left|p_{1} \frac{w_{3}}{w_{1}}+p_{4} \frac{w_{2}}{w_{4}}\right|^{2} \\
& +\left|p_{2} \frac{w_{3}}{w_{2}}+p_{4} \frac{w_{1}}{w_{4}}\right|^{2}+\left|p_{2} \frac{w_{4}}{w_{2}}+p_{3} \frac{w_{1}}{w_{3}}\right|^{2} .
\end{aligned}
$$




\subsection{Delta-flat directions}

We now show that there is always a radial trajectory, $\Phi=0$, along which $\partial W / \partial w_{i}$ is orthogonal to $w_{i}$ and lies in the null direction of $\hat{k}_{w}^{i \bar{j}}$. The term $\Delta V_{F}$ in (5.6) then vanishes and the prefactor $A$ of the superpotential becomes independent of the brane position. We call this a delta-flat direction. For the Ouyang embedding this trajectory was first found by Burgess et al. [18]. As noted in [18], delta-flat directions are noteworthy because they have $\Delta \eta=0$ and therefore imply a well-known no-go result for inflation [6].

More concretely, we see that for (5.7) to vanish one requires

$$
\Phi=0,
$$

i.e. at least one of the $w_{i}$ that enter the embedding must vanish. In fact, we notice that (5.7) vanishes whenever (5.8) does, so we can restrict our attention to (5.8). If any $p_{i}>1$, then we see immediately from the overall factor $\left|\Phi^{P}\right|^{2}=\left|w_{1}\right|^{2 p_{1}}\left|w_{2}\right|^{2 p_{2}}\left|w_{3}\right|^{2 p_{3}}\left|w_{4}\right|^{2 p_{4}}$ that (5.8) vanishes on $w_{i}=0$. For $p_{i} \leq 1$ there are only a few distinct cases: $\Phi=w_{1}, \Phi^{2}=w_{1} w_{2}$, $\Phi^{2}=w_{1} w_{3}, \Phi^{3}=w_{1} w_{2} w_{3}$, and $\Phi^{4}=w_{1} w_{2} w_{3} w_{4}$. In the next section we will illustrate the argument for the important case of the Ouyang embedding, $\Phi=w_{1}$; the proof is easily generalized to the remaining cases. This completes the proof that all ACR embeddings have delta-flat trajectories.

\subsection{Comparison of the Ouyang and Kuperstein Embeddings}

Recently the result of [9] has been applied $[18,33]$ to compactifications involving the Ouyang embedding $w_{1}=\mu$. In this case, the correction to the F-term potential is

$$
\Delta V_{F}=\frac{\kappa^{2}}{3 U^{2}}\left[\frac{3}{2}\left(\overline{W_{, \rho}} w_{1} W_{, w_{1}}+\text { c.c. }\right)+\frac{1}{\gamma} \hat{k}_{w}^{1 \overline{1}} W_{, w_{1}} \overline{W_{, w_{1}}}\right],
$$

where

$$
\hat{k}_{w}^{1 \overline{1}}=r\left(1+\frac{\left|w_{1}\right|^{2}}{2 r^{3}}-\frac{\left|w_{2}\right|^{2}}{r^{3}}\right) .
$$

There are two kinds of radial extremal trajectories: the delta-flat trajectory $w_{1}=w_{3}=w_{4}=$ 0 , for which $\Delta V_{F}=0$ [18], and also a trajectory $w_{2}=w_{3}=w_{4}=0, w_{1} \in \mathbb{R}$ used in [33].

The Kuperstein scenario is closely related to the Ouyang scenario except for two subtle differences which we now discuss:

1. There exists no delta-flat direction for the Kuperstein embedding.

2. The single-field potential along the non-delta-flat direction for the Ouyang embedding is identical in shape to that along the corresponding Kuperstein trajectory. However, 
the angular stability is different (see Appendix C). This trajectory in the Kuperstein embedding is stable for small $r$, while in the Ouyang embedding it is unstable in that regime.

To see this compare the correction to the F-term potential for the Ouyang embedding, (5.11), with the corresponding term for the Kuperstein embedding,

$$
\Delta V_{F}=\frac{\kappa^{2}}{3 U^{2}}\left[\frac{3}{2}\left(\overline{W_{, \rho}} z_{1} W_{, z_{1}}+\text { c.c. }\right)+\frac{1}{\gamma} k^{1 \overline{1}} W_{, z_{1}} \overline{W_{, z_{1}}}\right]
$$

where

$$
k^{1 \overline{1}}=r\left(1-\frac{1}{2} \frac{\left|z_{1}\right|^{2}}{r^{3}}\right) .
$$

This shows immediately that the Kuperstein embedding does not have a delta-flat direction, since $k^{1 \overline{1}}$ cannot vanish for $r>0$. This is to be viewed in contrast to the Ouyang case for which (5.12) vanishes on $w_{1}=0$.

Considering $k^{1 \overline{1}}, \hat{k}_{w}^{1 \overline{1}}$ for each case one may further show that the trajectories $2\left|z_{1}\right|^{2}=r^{3}$ and $\left|w_{1}\right|^{2}=r^{3}$ lead to identical shapes for the single-field potential. However, 'off-shell', i.e. away from the extremal path, $\hat{k}_{w}^{1 \overline{1}}$ for the Ouyang embedding is of a different form from $k^{1 \overline{1}}$ for the Kuperstein embedding. It is for this reason that the angular stability of the two scenarios is different (see Appendix $\mathrm{C}$ for details). In particular, while for the Kuperstein embedding the trajectory is stable for the regime of interest, for the Ouyang embedding it is unstable.

To discuss the issue of stability in simple terms, we consider $\theta_{1}=\theta_{2}=\theta$ and $\tilde{\psi} \equiv$ $\psi-\phi_{1}-\phi_{2}$, so that $w_{1}=e^{\frac{i}{2}} \tilde{\psi} r^{3 / 2} \sin ^{2}(\theta / 2)$. Then, as shown in [18] for $n=1$,

$$
V_{F}(\theta)=V_{1} \sin ^{2}(\theta / 2)+V_{2} \sin ^{4}(\theta / 2)+\text { const. },
$$

where

$$
\begin{aligned}
V_{1} & =\frac{\kappa^{2}\left|A_{0}\right|^{2} e^{-2 a \sigma}}{3 U^{2}} \frac{r}{\gamma \mu^{2}}\left(2-a \mu \gamma \sqrt{r} \cos \frac{\tilde{\psi}}{2}\left(9+4 a \sigma+6 W_{0} \frac{e^{a \sigma}}{\left|A_{0}\right|}\right)\right), \\
V_{2} & =\frac{1}{4} \frac{\kappa^{2}\left|A_{0}\right|^{2} e^{-2 a \sigma}}{3 U^{2}} \frac{r}{\gamma \mu^{2}}\left(-2+a \gamma r^{2}(12+8 a \sigma)\right) .
\end{aligned}
$$

We see that $\frac{\partial V_{F}}{\partial \theta}$ vanishes for $\theta=0$ or $\pi$.

The $\theta=0$ trajectory is delta-flat [18]. For this trajectory, $\frac{\partial^{2} V_{F}}{\partial \theta^{2}}=\frac{1}{2} V_{1}$ which is clearly positive for small $r$ and stays positive up to some critical radius $r_{c}$. To compute $r_{c}$ we evaluate $V_{1}$ at $\sigma=\sigma_{0}$ using

$$
4 a \sigma_{0}+9-6 W_{0} \frac{e^{a \sigma_{0}}}{\left|A_{0}\right|} \approx 3-4 s .
$$


We find

$$
V_{1} \approx \frac{\kappa^{2}\left|A_{0}\right|^{2} e^{-2 a \sigma_{0}}}{3 U^{2}} \frac{r}{\gamma \mu^{2}}\left(2+a \mu \gamma(4 s-3) \sqrt{r} \cos \frac{\tilde{\psi}}{2}\right) .
$$

For $s>3 / 4$ and for real positive $\mu$, the potential is minimized at $\tilde{\psi}=2 \pi$, where

$$
V_{1} \approx \frac{\kappa^{2}\left|A_{0}\right|^{2} e^{-2 a \sigma_{0}}}{3 U^{2}} \frac{r}{\gamma \mu^{2}}(2-a \mu \gamma(4 s-3) \sqrt{r}),
$$

This is positive as long as $r$ is less than $r_{c}$, where

$$
\frac{r_{c}}{r_{\mu}}=\frac{1}{(4 s-3)^{2}}\left(\frac{9}{a \sigma_{0}}\right)^{2} \frac{M_{P}^{4}}{\phi_{\mu}^{4}} .
$$

Applying the field range bound in the form $\frac{\phi_{\mu}^{2}}{M_{P}^{2}}<\frac{4}{N}$ one finds

$$
\frac{r_{c}}{r_{\mu}}>\frac{N^{2}}{4^{2}} \frac{1}{(4 s-3)^{2}}\left(\frac{9}{a \sigma_{0}}\right)^{2} .
$$

For typical parameters we therefore conclude that $r_{c} \geq r_{\mu}$ and the delta-flat direction is hence stable from the tip to at least the location $r_{\mu}$ of the D7-branes.

For the $\theta=\pi$ trajectory, $\frac{\partial^{2} V_{F}}{\partial \theta^{2}}=-\frac{1}{2} V_{1}$. This is negative for $r<r_{c}$ and the $\theta=\pi$ trajectory is therefore unstable in this regime. This analysis was carried out for $n=1$ but it illustrates the essential qualitative point (for a more general analysis with the same conclusion, see Appendix C.2).

In Appendix C.4 we show that for all ACR embeddings there are alternative trajectories with $\Phi \neq 0$ that are not delta-flat. This is important because it implies that, for a D3-brane moving along such a trajectory, $\eta$ can be different from $\frac{2}{3}$. We postpone a more general treatment of such trajectories for the future (but see Appendix C.4 for some preliminary remarks).

\section{Discussion}

In this section we will briefly take stock of our progress towards an explicit model of D-brane inflation. For this purpose, it is useful to consider the more general problem of deriving a low-energy Lagrangian from the data of a string compactification.

In principle, the data of a compactification - such as the background geometry, brane positions, and fluxes - determine the low-energy effective Lagrangian in full. In practice, one typically begins by deriving the leading-order effective Lagrangian, which follows from dimensional reduction of the classical ten-dimensional supergravity action, including the effect 
of fluxes but treating D-branes as probes. Then, one can include corrections to this action, including such things as nonperturbative terms in the superpotential, D-brane backreaction effects, string loop corrections to the Kähler potential, and $\alpha^{\prime}$ corrections to the Kähler potential. Except in cases with extended supersymmetry, it is typically impossible to obtain results beyond leading order in either series of corrections to the Kähler potential.

For the present purpose, an instructive way to organize these corrections is according to their effects on the slow-roll parameter $\eta$, as follows. The leading order classical fourdimensional Lagrangian we denote $\mathcal{L}_{0}$. Correction terms are well-known to give rise to inflaton masses of order $H$, and hence corrections of order unity to $\eta$. When all such effects, from any source whatsoever, have been added to $\mathcal{L}_{0}$, we denote this corrected Lagrangian $\mathcal{L}_{1}$. By definition, this is the Lagrangian whose inflaton mass term is a good approximation to the 'true' mass term that would follow from a dimensional reduction incorporating corrections of arbitrarily high degree. (Notice that the leading-order classical Lagrangian $\mathcal{L}_{0}$ may itself contain large inflaton mass terms.) Finally, if to $\mathcal{L}_{1}$ we add the leading terms that give corrections to $\eta$ that are parametrically small compared to unity, we call the resulting Lagrangian $\mathcal{L}_{2}$. In sum, we propose to organize corrections to the Lagrangian according to the degree of their effects on $\eta$, even though such an organization does not correspond to a literal expansion parameter such as the string coupling.

To determine whether a given model gives rise to prolonged slow-roll inflation, one needs to know $\mathcal{L}_{1}$. However, it is rarely true that all the required results are available. For example, in the warped brane inflation model of [6], the inflaton-dependence of the threshold factor $A(\phi)$ of the nonperturbative superpotential was not known until recently [11, 9]. Similarly, in Kähler moduli inflation ( $c f$. the second reference in [7]), a particular term in the Kähler potential that could give $\Delta \eta \sim 1$ has not yet been computed (though it has been conjectured that this term might vanish.) Although such partial data is generally insufficient to determine whether a model is successful, even this degree of detail is relatively rare: a fair fraction of proposed models of string inflation include only the $\mathcal{L}_{0}$ data, without any corrections at all.

In this paper, we have progressed towards a full understanding of the Lagrangian $\mathcal{L}_{1}$ for the warped brane inflation models of [6]. However, as we will now explain, further work is necessary.

First, let us briefly recall the best-understood correction terms. The D3-brane potential receives contributions from the mixing between the volume and the D3-brane position in the DeWolfe-Giddings Kähler potential (2.9). Moreover, the nonperturbative superpotential receives the correction (2.15) sourced by the backreaction of the D3-brane on the warp factor. Holomorphy of the gauge kinetic function ensures that this correction, which corresponds to a one-loop threshold factor, is the only perturbative correction to the superpotential. The only additional contributions to the superpotential come from multi-instantons, which give a negligibly small effect. 
The Kähler potential, however, is not protected by holomorphy, and in general receives $\alpha^{\prime}$ and $g_{s}$ corrections. In the large-volume, weak-coupling limit, these corrections are suppressed relative to the leading terms in the DeWolfe-Giddings Kähler potential, and so generate mass terms that are generically smaller than $H$ by powers of the inverse volume or powers of the string coupling. Hence, by our above definition, these corrections correspond to terms in $\mathcal{L}_{2}$. Although complete results for these terms are not available for a general compactification, the work of Berg, Haack, and Körs [39, 40] in the toroidal orientifold case gives substantial guidance. These authors found that the leading D3-brane-dependent corrections to $\mathcal{K}$ are of two types, one suppressed by an additional power of $\rho$ compared to the DeWolfe-Giddings result, and the other suppressed by one power of $g_{s}$. For $\rho \gg 1, g_{s} \ll 1$, these terms give a parametrically small correction to the D3-brane potential, and so belong to what we have called $\mathcal{L}_{2}$. Even better, in some cases [39] the expectations of naive dimensional analysis are borne out, and the numerical prefactors of these higher-order terms are moderately small. ${ }^{13}$

Corrections due to the fluxes are a further possibility. The best-understood $\alpha^{\prime}$ corrections arise from the term $\left(\alpha^{\prime}\right)^{3} R^{4}$ in ten dimensions, with $R^{4}$ standing for an appropriate contraction of four powers of the Riemann tensor. In the presence of three-form flux $G_{3}$, there are additional terms from $\left(G_{3}\right)^{2} R^{3}$, and it is less clear how these correct the Kähler potential [41]. However, it has been argued that at large volume, hence low flux density, this effect is subleading [42].

Finally, and perhaps most importantly, perturbations of the background fields in the throat, which can arise from sources in the bulk, can give substantial corrections to the D3-brane potential. ${ }^{14}$ It was argued in [43] (see also [33]) that in special cases these effects may be small compared to the forces from the D7-brane. However, lacking a more complete understanding of these effects, we do not claim that the potential we have presented is completely general. Instead, our construction is representative of a particularly tractable class of situations in which the bulk effects are small.

\section{Conclusions}

In this paper we have systematically studied the potential for a D3-brane in a warped throat containing holomorphically-embedded D7-branes. As explained in [6], this system is a promising candidate for an explicit model of inflation in string theory. However, the warped brane inflation model of [6] is well-known to suffer from an inflaton mass problem, in that corrections from moduli stabilization tend to curve the potential and make slowroll inflation impossible. The true severity of this problem - and, correspondingly, the

\footnotetext{
${ }^{13}$ We thank M. Berg for helpful discussions of this point.

${ }^{14}$ We thank S. Kachru for explanations of this point.
} 
status of the model - have remained unclear, because the functional form of one particular correction term, arising from a threshold correction to the nonperturbative superpotential, was unavailable before the recent result of [9]. In this work, building on [9], we have studied the corrected potential in detail. This equipped us to assess the true status of the warped brane inflation model [6] and to ascertain whether prolonged slow-roll inflation is indeed possible.

Our method for analyzing the potential involved several nontrivial improvements over existing approximations. First, we systematically identified stable minima in the angular directions of the conifold, and showed how the radial potential depends on the choice of angular minimum. Second, we showed that the common assumption that the compactification volume remains stabilized at its minimum during inflation is inadequate: the volume shrinks slightly as the D3-brane falls down the throat, and this leads to non-negligible corrections to the effective D3-brane potential. We gave an analytic expression for the volume as a function of the D3-brane position and showed that this is an excellent approximation to the full result.

For a large class of embeddings of the wrapped branes, the ACR embeddings, we showed that the radial potential for a D3-brane at a particular angular extremum is necessarily too steep to support inflation, because the contributions computed in [9] vanish along the trajectory, and so the no-go result of [6] applies. This was first explained in [18] for a special ACR embedding, the Ouyang embedding [19]. Our results here generalize this to the full ACR class. It follows that these trajectories in throats containing wrapped branes with ACR embeddings do not permit slow roll D-brane inflation, even if one allows an arbitrary degree of fine-tuning: there is simply no parameter that can be varied to flatten the potential in such a case. However, we also showed that all ACR embeddings have alternative trajectories, corresponding to other choices of the angular extremum, for which this no-go result does not apply.

Our main result was an analysis of a very simple and symmetric embedding, the $\mathrm{Ku}-$ perstein embedding, that does allow a flat D3-brane potential. We found that for certain fine-tuned ranges of the compactification parameters, the potential is flat enough to allow prolonged inflation. However, the resulting potential is not as simple as that conjectured in [6] and further elaborated in [13]: moduli stabilization gives rise to a potential that is much more complicated than a mass term for radial motion. Furthermore, adjusting the potential by varying microscopic parameters changes features in the potential instead of just rescaling the mass term. In particular, one can fine-tune to arrange for a flat region suitable for inflation, but we found that this will occur around an inflection point away from the origin. Hence, when inflation occurs, it does so near an approximate inflection point, rather than in a shallow quadratic potential centered on the origin. In short, we found that a low-order Taylor expansion of the potential around the minimum at the tip of the throat 
does not properly describe those regions of the potential where inflation is possible. Instead, one is obliged to use the complete potential presented here. Our result implies that the phenomenology of some classes of warped D-brane inflation models is well-described by an effective single-field potential with a constant and cubic term. As we explained, models of this sort (see [36] for an analogous example in the MSSM) are particularly sensitive to the initial conditions. Moreover, the tilt of the scalar spectrum is exquisitely sensitive to the slope of the potential near the approximate inflection point.

One important, general lesson of our work is that there is considerably less freedom to adjust the parameters of this system than one might expect from the low-energy effective action. First, the functional form of $A(\phi)$ derived in [9] is rather special: most importantly, $A(\phi)$ contains no quadratic term, and does not lead to any new quadratic terms in the inflaton potential. This implies that the potential cannot be flattened uniformly, and $\eta$ can only be

small in a limited region. Second, the range of $\phi$ is limited by the microscopic constraint of [21]. Finally, the field range is linked to the scale of inflation, because of a new geometric constraint linking the size of the wrapped four-cycle and the length of the throat. Using these results, we found that although this system depends on many microscopic parameters, in many cases it is nevertheless impossible to choose these parameters in such a way that the slow-roll parameter $\eta$ is fine-tuned to vanish. This occurs because constraints originating in the geometry of the compactification correlate the microscopic quantities, so that the true number of adjustable parameters is much smaller than a naive estimate would suggest.

An important direction for future work is a more comprehensive understanding of any additional corrections to the potential, such as $\alpha^{\prime}$ corrections, string loop corrections, and perturbations of the throat metric due to bulk sources. We have argued that in some cases the presence of such effects is not fatal for inflation, but precise observational predictions will certainly depend on these effects.

Finally, there is a pressing need for a more natural model of string inflation than the one we have presented here.

\section{Acknowledgments}

We are grateful to Paul Steinhardt for collaboration on the companion paper [1] and for many useful discussions of this paper. It is a pleasure to thank Marcus Berg, Cliff Burgess, Jim Cline, Joe Conlon, Oliver DeWolfe, Steve Giddings, Shamit Kachru, Renata Kallosh, Justin Khoury, Juan Maldacena, Arvind Murugan, Enrico Pajer, Sudhakar Panda, Joe Polchinski, Gary Shiu, Eva Silverstein, Shinji Tsujikawa, Henry Tye, Bret Underwood, and Herman Verlinde for many useful discussions and insightful comments. We especially thank Shamit Kachru for detailed comments on a draft. D.B. thanks Julian Baumann for assistance with 
Figure 1. D.B. thanks Dragan Huterer, Hiranya Peiris, and the KICP, Chicago for their hospitality while this work was completed. A.D. would like to thank the Galileo Galilei Institute for Theoretical Physics, the Perimeter Institute, and the University of Chicago for their hospitality. The research of A.D. was supported in part by Grant RFBR 07-0200878, and Grant for Support of Scientific Schools NSh-8004.2006.2. L.M. is grateful to the KITP Santa Barbara and the theory groups at the Perimeter Institute, the University of Pennsylvania, the University of Wisconsin, Cornell, Stanford, the University of Texas, the University of Michigan, Texas A\&M, Harvard, and Yale for their hospitality. This research was supported in part by the National Science Foundation under Grant No. PHY-0243680 and by the Department of Energy under grant DE-FG02-90ER40542. Any opinions, findings, and conclusions or recommendations expressed in this material are those of the authors and do not necessarily reflect the views of these funding agencies. 


\section{A Background Geometry}

\section{A.1 The singular conifold}

The singular conifold is a non-compact Calabi-Yau threefold defined by the constraint equation $[24]$

$$
\sum_{i=1}^{4} z_{i}^{2}=0
$$

where $\left\{z_{i} ; i=1,2,3,4\right\}$ are complex coordinates in $\mathbb{C}^{4}$. The conifold constraint (A.1) may be written as $\operatorname{det}(W)=0$ where

$$
W=\frac{1}{\sqrt{2}}\left(\begin{array}{cc}
z_{3}+i z_{4} & z_{1}-i z_{2} \\
z_{1}+i z_{2} & -z_{3}+i z_{4}
\end{array}\right) \equiv\left(\begin{array}{cc}
w_{3} & w_{2} \\
w_{1} & w_{4}
\end{array}\right) .
$$

The Kähler potential (2.3) is chosen such that the Kähler metric on the conifold, $k_{\alpha \bar{\beta}} \equiv$ $\partial_{\alpha} \partial_{\bar{\beta}} k$, is Calabi-Yau (Ricci-flat)

$$
\begin{aligned}
\mathrm{d} s^{2} & =\partial_{\alpha} \partial_{\bar{\beta}} k \mathrm{~d} u^{\alpha} \mathrm{d} \overline{u^{\beta}} \\
& =k^{\prime \prime}\left|\operatorname{Tr}\left(W^{\dagger} \mathrm{d} W\right)\right|^{2}+k^{\prime} \operatorname{Tr}\left(\mathrm{d} W \mathrm{~d} W^{\dagger}\right),
\end{aligned}
$$

where $(\ldots)^{\prime} \equiv \frac{d(\ldots)}{d r^{3}}$ and $\left\{u_{\alpha} ; \alpha=1,2,3\right\}$ are three complex coordinates on the conifold, e.g. $u_{\alpha}=z_{\alpha}$. The metric on the conifold (A.3) may be cast in the form (2.2) where

$$
\mathrm{d} s_{T^{1,1}}^{2}=\frac{1}{9}\left(\mathrm{~d} \psi+\sum_{i=1}^{2} \cos \theta_{i} \mathrm{~d} \phi_{i}\right)^{2}+\frac{1}{6} \sum_{i=1}^{2}\left(\mathrm{~d} \theta_{i}+\sin ^{2} \theta_{i} \mathrm{~d} \phi_{i}^{2}\right)
$$

is the metric on the Einstein space $T^{1,1}$. The complex coordinates $z_{i}$ are related to the real coordinates $\left\{r \in[0, \infty], \theta_{i} \in[0, \pi], \phi_{i} \in[0,2 \pi], \psi \in[0,4 \pi]\right\}$ via

$$
\begin{aligned}
& z_{1}=\frac{r^{3 / 2}}{\sqrt{2}} e^{\frac{i}{2} \psi}\left[\cos \left(\frac{\theta_{1}+\theta_{2}}{2}\right) \cos \left(\frac{\phi_{1}+\phi_{2}}{2}\right)+i \cos \left(\frac{\theta_{1}-\theta_{2}}{2}\right) \sin \left(\frac{\phi_{1}+\phi_{2}}{2}\right)\right], \\
& z_{2}=\frac{r^{3 / 2}}{\sqrt{2}} e^{\frac{i}{2} \psi}\left[-\cos \left(\frac{\theta_{1}+\theta_{2}}{2}\right) \sin \left(\frac{\phi_{1}+\phi_{2}}{2}\right)+i \cos \left(\frac{\theta_{1}-\theta_{2}}{2}\right) \cos \left(\frac{\phi_{1}+\phi_{2}}{2}\right)\right], \\
& z_{3}=\frac{r^{3 / 2}}{\sqrt{2}} e^{\frac{i}{2} \psi}\left[-\sin \left(\frac{\theta_{1}+\theta_{2}}{2}\right) \cos \left(\frac{\phi_{1}-\phi_{2}}{2}\right)+i \sin \left(\frac{\theta_{1}-\theta_{2}}{2}\right) \sin \left(\frac{\phi_{1}-\phi_{2}}{2}\right)\right], \\
& z_{4}=\frac{r^{3 / 2}}{\sqrt{2}} e^{\frac{i}{2} \psi}\left[-\sin \left(\frac{\theta_{1}+\theta_{2}}{2}\right) \sin \left(\frac{\phi_{1}-\phi_{2}}{2}\right)-i \sin \left(\frac{\theta_{1}-\theta_{2}}{2}\right) \cos \left(\frac{\phi_{1}-\phi_{2}}{2}\right)\right] .
\end{aligned}
$$


The complex coordinates $w_{i}$ are related to the real coordinates $\left\{r, \theta_{i}, \phi_{i}, \psi\right\}$ via

$$
\begin{aligned}
& w_{1}=r^{3 / 2} e^{\frac{i}{2}\left(\psi-\phi_{1}-\phi_{2}\right)} \sin \frac{\theta_{1}}{2} \sin \frac{\theta_{2}}{2}, \\
& w_{2}=r^{3 / 2} e^{\frac{i}{2}\left(\psi+\phi_{1}+\phi_{2}\right)} \cos \frac{\theta_{1}}{2} \cos \frac{\theta_{2}}{2}, \\
& w_{3}=r^{3 / 2} e^{\frac{i}{2}\left(\psi+\phi_{1}-\phi_{2}\right)} \cos \frac{\theta_{1}}{2} \sin \frac{\theta_{2}}{2}, \\
& w_{4}=r^{3 / 2} e^{\frac{i}{2}\left(\psi-\phi_{1}+\phi_{2}\right)} \sin \frac{\theta_{1}}{2} \cos \frac{\theta_{2}}{2} .
\end{aligned}
$$

\section{A.2 Making $S O(4)$ symmetry manifest}

It is sometimes convenient to write the F-term potential (2.13) in terms of the four homogeneous coordinates $z_{i}$ of the embedding space $\mathbb{C}^{4}$ which makes the $S O(4)$ symmetry of the conifold transparent. For that reason we define a new metric $\widehat{\mathcal{K}}^{A \bar{B}}$ which depends on $z_{i}$ in such a way that for any function $W\left(z_{i}\right)$ the following identity is satisfied

$$
D_{A} W \widehat{\mathcal{K}}^{A \bar{B}} \overline{D_{B} W} \equiv D_{\Sigma} W \mathcal{K}^{\Sigma \bar{\Omega}} \overline{D_{\Omega} W}
$$

where $\left\{Z^{A}\right\} \equiv\left\{\rho, z_{i} ; i=1,2,3,4\right\}$ and $\left\{Z^{\Sigma}\right\} \equiv\left\{\rho, z_{\alpha} ; \alpha=1,2,3\right\}$. In this equation the conifold constraint, $z_{4}^{2}=z_{4}^{2}\left(z_{\alpha}\right)=-\sum_{\alpha=1}^{3} z_{\alpha}^{2}$, is substituted after differentiation on the left-hand side and before differentiation on the right-hand side. The metric $\widehat{\mathcal{K}}^{A \bar{B}}\left(z_{i}\right)$ defined through (A.13) is not unique and the choice of one over another is a matter of convenience. We construct $\widehat{\mathcal{K}}^{A \bar{B}}$ with the help of the auxiliary matrix $J^{A}{ }_{\Sigma}$

$$
\widehat{\mathcal{K}}^{A \bar{B}}=J_{\Sigma}^{A} \mathcal{K}^{\Sigma \bar{\Omega}} J^{\bar{B}} \bar{\Omega},
$$

where $J^{A}$ is defined as follows

$$
D_{\Sigma} W=\frac{\partial Z^{A}}{\partial Z^{\Sigma}} D_{A} W \equiv J_{\Sigma}^{A} D_{A} W, \quad J_{\Sigma}^{A}=\left(\begin{array}{c|c}
1 & 0 \\
\hline 0 & \frac{\delta_{i \alpha}}{\sqrt{-\sum_{\gamma}^{3} z_{\gamma}^{2}}}
\end{array}\right) .
$$

This gives $\widehat{\mathcal{K}}^{A \bar{B}}$ as a function of $z_{\alpha}$. To find it as a function of $z_{i}$ guess a $\widehat{\mathcal{K}}^{A \bar{B}}\left(z_{i}\right)$ such that it reduces to $\widehat{\mathcal{K}}^{A \bar{B}}\left(z_{\alpha}\right)$ after substituting the conifold constraint. This step and hence $\widehat{\mathcal{K}}^{A \bar{B}}\left(z_{i}\right)$ is not unique. Nevertheless finding an $S O(4)$-invariant $\widehat{\mathcal{K}}^{A \bar{B}}\left(z_{i}\right)$ is not difficult, e.g. replacing $\left(-\sum_{\gamma=1}^{3} z_{\gamma}^{2}\right)^{1 / 2}$ by $z_{4}$ everywhere in $\widehat{\mathcal{K}}^{A \bar{B}}\left(z_{\alpha}\right)$ and $J^{A}{ }_{\Sigma}$ we find

$$
\widehat{\mathcal{K}}^{A \bar{B}}=\frac{\kappa^{2} U}{3}\left(\begin{array}{c|c}
U+\gamma k_{l} \hat{k}^{l \bar{m}} k_{\bar{m}} & k_{l} \hat{k}^{l \bar{\jmath}} \\
\hline \hat{k}^{i \bar{m}} k_{\bar{m}} & \frac{1}{\gamma} \hat{k}^{i \bar{\jmath}}
\end{array}\right),
$$


where

$$
k_{i}=\frac{\overline{z_{i}}}{r}
$$

and

$$
\hat{k}^{i \bar{\jmath}}=J_{\alpha}^{i} k^{\alpha \bar{\beta}} J_{\bar{\beta}}^{\bar{\jmath}}=r\left[\delta^{i \bar{\jmath}}+\frac{1}{2} \frac{z_{i} \overline{z_{j}}}{r^{3}}-\frac{\overline{z_{i}} z_{j}}{r^{3}}\right] .
$$

Notice that $\hat{k}^{i \bar{\jmath}}$ is not the inverse of $k_{i \bar{\jmath}}=\frac{1}{r}\left[\delta_{i \bar{\jmath}}-\frac{1}{3} \frac{\overline{z_{i}} z_{j}}{r^{3}}\right]$, which is $k^{i \bar{\jmath}}=r\left[\delta^{i \bar{\jmath}}+\frac{1}{2} \frac{z_{i} \overline{z_{j}}}{r^{3}}\right]$. From (A.17) and (A.18) one then finds

$$
k_{l} \hat{k}^{l \bar{\jmath}}=\frac{3}{2} \bar{z}^{j}, \quad k_{l} \hat{k}^{l \bar{m}} k_{\bar{m}}=\frac{3}{2} r^{2}=\hat{r}^{2}=k,
$$

and hence,

$$
\widehat{\mathcal{K}}^{A \bar{B}}=\frac{\kappa^{2} U}{3}\left(\begin{array}{c|c}
\rho+\bar{\rho} & \frac{3}{2} \overline{z_{j}} \\
\hline \frac{3}{2} z_{i} & \frac{r}{\gamma}\left[\delta^{i \bar{\jmath}}+\frac{1}{2} \frac{z_{i} \overline{z_{j}}}{r^{3}}-\frac{\overline{z_{i}} z_{j}}{r^{3}}\right]
\end{array}\right) .
$$

Using the above results we arrive at the F-term potential

$$
V_{F}=\frac{\kappa^{2}}{3 U^{2}}\left[(\rho+\bar{\rho})\left|W_{, \rho}\right|^{2}-3\left(\bar{W} W_{, \rho}+\text { c.c. }\right)+\frac{3}{2}\left(\overline{W_{, \rho}} z^{i} W_{, i}+\text { c.c. }\right)+\frac{1}{\gamma} \hat{k}^{i \jmath} W_{, i} \overline{W_{, j}}\right] .
$$

The result (A.21) is essential for the main analysis presented in this paper. In terms of the $w_{i}$-coordinates the F-term potential (A.21) is

$$
V_{F}=\frac{\kappa^{2}}{3 U^{2}}\left[(\rho+\bar{\rho})\left|W_{, \rho}\right|^{2}-3\left(\bar{W} W_{, \rho}+\text { c.c. }\right)+\frac{3}{2}\left(\overline{W_{, \rho}} w^{i} W_{, i}+c . c .\right)+\frac{1}{\gamma} \hat{k}_{w}^{i \bar{\gamma}} W_{, i} \overline{W_{, j}}\right],
$$

where

$$
\hat{k}_{w}^{i \bar{\jmath}}=r\left[\delta^{i \bar{\jmath}}+\frac{1}{2} \frac{w_{i} \overline{w_{j}}}{r^{3}}-\frac{c_{i}^{i^{\prime}} c_{j}^{j^{\prime}} \overline{w_{i^{\prime}}} w_{j^{\prime}}}{r^{3}}\right] .
$$

The matrix $c_{j}^{i^{\prime}}$ has only four non-zero elements $c_{2}^{1}=c_{1}^{2}=1$ and $c_{4}^{3}=c_{3}^{4}=-1$.

\section{B Dimensional Reduction}

As explained in [9] and reviewed in $\S 2$ of this paper, D7-branes wrapping certain four-cycles in the compactification source nonperturbative effects that stabilize the volume modulus. In $§$ B. 1 we identify the real part of the Kähler modulus with the warped volume of the four-cycle and give a detailed derivation of the DeWolfe-Giddings Kähler potential. These results are well known, but our goal here is to fix notation with enough care to permit precise discussions elsewhere in the paper. $\S \mathrm{B} .2$ computes the throat contribution to the warped four-cycle volume for our setup and relates it to microscopic compactification data. We also explain how this relates microscopic input to the energy scale of inflation. Finally, in $\S$ B.3, we present an improved derivation of the field range bound of [21] that includes a non-trivial breathing mode of the compactification. 


\section{B.1 Kähler modulus and Kähler potential}

We consider the line element

$$
\mathrm{d} s^{2}=h^{-1 / 2}(y) e^{-6 u} g_{\mu \nu} \mathrm{d} x^{\mu} \mathrm{d} x^{\nu}+h^{1 / 2}(y) e^{2 u} g_{\alpha \bar{\beta}} \mathrm{d} y^{\alpha} \mathrm{d} \overline{y^{\beta}},
$$

where $g_{\mu \nu}$ is the four-dimensional Einstein frame metric, $h$ is the warp factor, $g_{\alpha \bar{\beta}}$ is a fiducial metric on the internal space, and the factor $e^{u}$ extracts the breathing mode.

Before proceeding, let us explain the division of the metric $\tilde{g}_{\alpha \bar{\beta}} \equiv e^{2 u} g_{\alpha \bar{\beta}}$ into a fiducial metric $g$ and a breathing mode $e^{2 u}$. These two objects do play different roles: note in particular that although $g$ affects the four-dimensional Planck mass

$$
M_{P}^{2}=\frac{1}{\pi}\left(T_{3}\right)^{2} \int \mathrm{d}^{6} y \sqrt{g} h \equiv \frac{1}{\pi}\left(T_{3}\right)^{2} V_{6}^{w}
$$

the breathing mode does not, because of the factor $e^{-6 u}$ in the spacetime term of (B.1).

For any fixed location $Y \equiv Y_{\alpha}$ of the D3-brane, there is a minimum $\rho_{\star}(Y)$ of the nonperturbatively-generated potential for the Kähler modulus $\rho$. (We will soon come to a precise definition of $\rho$ in terms of the fields in (B.1).) We argued in $\S 3$ for an adiabatic approximation in which, as the D3-brane moves and hence the location $\rho_{\star}(Y)$ of the instantaneous minimum changes, $\rho$ moves to remain in this instantaneous minimum $\rho_{\star}(Y)$. In terms of the fields in (B.1), this is most conveniently represented ${ }^{15}$ by fixing the fiducial metric once and for all, but allowing the breathing mode $e^{u(Y, \bar{Y})}$ to keep track of the change in the volume that is due to the displacement of the D3-brane.

To this end, we normalize the fiducial metric $g$ to correspond to $\rho_{\star}(0)$, i.e. so that the volume computed with $g$ is precisely the physical volume of the internal space when the D3brane sits at the tip of the throat, $Y \approx 0$. Then, $e^{u(Y, \bar{Y})}$ represents the change in the volume that is due to the displacement of the D3-brane away from the tip, and by this definition, $u(0)=0$. In the throat region, the fiducial metric takes the form

$$
g_{\alpha \bar{\beta}} \mathrm{d} y^{\alpha} \mathrm{d} \overline{y^{\beta}}=\mathrm{d} \hat{r}^{2}+\hat{r}^{2} \mathrm{~d} s_{T^{1,1}}^{2} .
$$

The nonperturbative superpotential arising from strong gauge dynamics on $n$ D7-branes (or Euclidean D3-instantons) wrapping a four-cycle $\Sigma_{4}$ in the background (B.1) is [9]

$$
\left|W_{\mathrm{np}}\right|^{2} \propto \exp \left[-\frac{2 T_{3} V_{\Sigma_{4}}^{w} e^{4 u}}{n}\right]
$$

where $V_{\Sigma_{4}}^{w} \equiv \int \mathrm{d}^{4} \xi \sqrt{g^{\text {ind }}} h$. Before the mobile D3-brane enters the throat region, the (warped) four-cycle volume is $\left(V_{\Sigma_{4}}^{w}\right)_{0}$. When the D3-brane is in the throat, it induces a

\footnotetext{
${ }^{15}$ At the end of this section we will verify that choosing a different normalization of the fiducial metric does not affect any physical quantities.
} 
perturbation in the warp factor, $\delta h$, which sources a change in the warped four-cycle volume, $\delta V_{\Sigma_{4}}^{w}(Y)$. (This change in the warped volume is logically distinct from the shift in the stabilized value of the Kähler modulus studied in Appendix D.) Hence,

$$
\begin{aligned}
\left|W_{\mathrm{np}}\right|^{2} & \propto \exp \left[-\frac{2 T_{3} \delta V_{\Sigma_{4}}^{w}(Y) e^{4 u(Y, \bar{Y})}}{n}\right] \exp \left[-\frac{2 T_{3}\left(V_{\Sigma_{4}}^{w}\right)_{0} e^{4 u(Y, \bar{Y})}}{n}\right] \\
& \equiv|A(Y)|^{2} e^{-a(\rho+\bar{\rho})}
\end{aligned}
$$

We now define $a \equiv \frac{2 \pi}{n}$ and identify the Kähler modulus [9]

$$
\rho+\bar{\rho} \equiv \Gamma e^{4 u(Y, \bar{Y})}+\gamma k(Y, \bar{Y}), \quad \Gamma \equiv \frac{T_{3}\left(V_{\Sigma_{4}}^{w}\right)_{0}}{\pi} .
$$

Here $k$ is the little Kähler potential for the fiducial metric on the conifold, $\partial_{\alpha} \partial_{\bar{\beta}} k \equiv g_{\alpha \bar{\beta}}$. The term proportional to $k$ in (B.7) has to be added to make $\rho$ holomorphic; see [12, 9] for extensive discussions of this issue. Because $u(0)=k(0)=0$, we may relate the parameter $\Gamma$ to the value of the stabilized Kähler modulus $\rho$ when the D3-brane is at the tip of the throat,

$$
\Gamma \equiv \rho_{\star}(0)+\bar{\rho}_{\star}(0)=2 \sigma_{\star}(0) \equiv 2 \sigma_{0} .
$$

The range of allowed values for $\sigma_{0}$ is constrained by the throat contribution to the warped four-cycle volume, which we will compute in the next section.

With these definitions the DeWolfe-Giddings Kähler potential (see footnote 5 for comments on possible corrections to this Kähler potential) is

$$
\mathcal{K}=-3 M_{P}^{2} \log [\rho+\bar{\rho}-\gamma k(Y, \bar{Y})]=-3 M_{P}^{2} \log \left[\Gamma e^{4 u(Y, \bar{Y})}\right] .
$$

To determine the constant $\gamma$, we compare the kinetic terms derived from the DBI action,

$$
\mathcal{L}_{\text {kin }}^{\text {DBI }}=-\frac{1}{2} T_{3} e^{-4 u} g_{\alpha \bar{\beta}} \partial_{\mu} Y^{\alpha} \partial^{\mu} \overline{Y^{\beta}},
$$

with the kinetic terms ${ }^{16}$ derived from (B.9),

$$
\begin{aligned}
\mathcal{L}_{\text {kin }}^{\mathcal{K}} & =-\mathcal{K}_{\alpha \bar{\beta}} \partial_{\mu} Y^{\alpha} \partial^{\mu} \overline{Y^{\beta}} \\
& \approx 3 M_{P}^{2} e^{-4 u} \partial_{\alpha} \partial_{\bar{\beta}} e^{4 u} \partial_{\mu} Y^{\alpha} \partial^{\mu} \overline{Y^{\beta}},
\end{aligned}
$$

$$
\begin{aligned}
& { }^{16} \text { Of course, the complete kinetic terms for the D3-brane coordinates and the volume modulus are } \\
& \qquad \begin{aligned}
\mathcal{L}_{\text {kin }} \equiv & -\mathcal{K}_{\rho \bar{\rho}} \partial_{\mu} \rho \partial^{\mu} \bar{\rho}-\mathcal{K}_{Y_{\alpha} \overline{Y_{\beta}}} \partial_{\mu} Y^{\alpha} \partial^{\mu} \overline{Y^{\beta}}-\left(\mathcal{K}_{\rho \overline{Y_{\beta}}} \partial_{\mu} \rho \partial^{\mu} \overline{Y^{\beta}}+\text { c.c. }\right) \\
= & 3 M_{P}^{2} \frac{\gamma k_{\alpha \bar{\beta}} \partial_{\mu} Y^{\alpha} \partial^{\mu} \overline{Y^{\beta}}}{U} \\
& -3 M_{P}^{2}\left|\frac{\partial_{\mu} \rho}{U}\right|^{2}+3 M_{P}^{2}\left|\frac{\gamma k_{\alpha} \partial_{\mu} \overline{Y^{\alpha}}}{U}\right|^{2}+3 M_{P}^{2}\left(\frac{\partial_{\mu} \rho}{U} \frac{\gamma k_{\bar{\beta}} \partial^{\mu} \overline{Y^{\beta}}}{U}+\text { c.c. }\right) \\
\approx & \frac{3 M_{P}^{2} \gamma k_{\alpha \bar{\beta}} \partial_{\mu} Y^{\alpha} \partial^{\mu} \overline{Y^{\beta}}}{U}
\end{aligned}
\end{aligned}
$$


where

$$
\partial_{\alpha} \partial_{\bar{\beta}} e^{4 u}=-\frac{\gamma}{\Gamma} \partial_{\alpha} \partial_{\bar{\beta}} k=-\frac{\gamma}{\Gamma} g_{\alpha \bar{\beta}}
$$

Equations (B.10) and (B.11) are consistent if we define

$$
\gamma \equiv \frac{\Gamma}{6} \frac{T_{3}}{M_{P}^{2}}=\frac{1}{6} \frac{\left(V_{\Sigma_{4}}^{w}\right)_{0}}{V_{6}^{w}} .
$$

Using equation (B.8) this may be written in the useful form

$$
\gamma=\frac{\sigma_{0}}{3} \frac{T_{3}}{M_{P}^{2}}
$$

We now notice that the physical quantities of interest, such as $\rho$ and $\gamma k$, are independent of the split into breathing mode $e^{u(0)}$ and fiducial metric $g$ in (B.1). For example,

$$
\gamma k=\frac{1}{6} \frac{T_{3}}{\left[M_{P}^{2} e^{6 u}\right]} \frac{T_{3}\left[\left(V_{\Sigma_{4}}^{w}\right)_{0} e^{4 u}\right]}{\pi}\left[e^{2 u} k\right]=\frac{1}{6} \frac{\left(\tilde{V}_{\Sigma_{4}}^{w}\right)_{0}}{\tilde{V}_{6}^{w}} \tilde{k} \equiv \tilde{\gamma} \tilde{k},
$$

where we have defined

$$
\tilde{k} \equiv e^{2 u} k=e^{2 u} r^{2}=\tilde{r}^{2},
$$

and

$$
\tilde{\gamma} \equiv e^{-2 u} \gamma=\frac{\tilde{\Gamma}}{6} \frac{T_{3}}{\left[M_{P}^{2} e^{6 u}\right]}=\frac{1}{6} \frac{\left(\tilde{V}_{\Sigma_{4}}^{w}\right)_{0}}{\tilde{V}_{6}^{w}}
$$

where

$$
\tilde{\Gamma} \equiv \Gamma e^{4 u}=2 \sigma_{0} e^{4 u}
$$

This shows that $\tilde{\gamma} \tilde{k}$ is invariant under the change of conventions

$$
e^{2 u(0)} \rightarrow \lambda e^{2 u(0)}, \quad g_{\alpha \bar{\beta}} \rightarrow \lambda^{-1} g_{\alpha \bar{\beta}}
$$

One easily sees that $\rho+\bar{\rho}$ is likewise invariant. We have therefore justified our original choice of the convenient value $u(0)=0$.

In the final relation we have focused attention on a subset of the kinetic terms for the D3-brane coordinates. This is justified for several reasons. First, in this paper we are specifically searching for (fine-tuned) configurations in which the D3-brane potential is unusually flat. In such a case, it is consistent to use an adiabatic approximation for the motion of the volume modulus, and omit the kinetic terms for $\rho$. Next, the term involving $\left|k_{\alpha} \partial_{\mu} \overline{Y^{\alpha}}\right|^{2}$ is suppressed, relative to the term we have retained, by $U^{-1} \ll 1$. Furthermore, $k_{\alpha}$ vanishes at the tip of a singular conifold, and is correspondingly very small at the tip of the deformed conifold we are considering. Finally, from the explicit form of $k$ we learn that the term $\left|k_{\alpha} \partial_{\mu} \overline{Y^{\alpha}}\right|^{2}$ is suppressed by the small quantity $\left(\phi / M_{P}\right)^{2}$ relative to the term we have retained. 


\section{B.2 Warped four-cycle volume}

The four-cycle $\Sigma_{4}$ wrapped by the D7-branes is, in principle, compact. However, we have access to explicit metric information only in the throat region, but can still make progress by deriving results that are largely independent of the unknown bulk region. The fourcycle volume receives contributions both from the throat region and the bulk, $\left(V_{\Sigma_{4}}^{w}\right)_{0}=$ $\left(V_{\Sigma_{4}}^{w}\right)_{0, \text { throat }}+\left(V_{\Sigma_{4}}^{w}\right)_{0 \text {,bulk }}$. In the remainder of this section we compute $\left(V_{\Sigma_{4}}^{w}\right)_{0, \text { throat }}$ for various embeddings and use $\left(V_{\Sigma_{4}}^{w}\right)_{0} \equiv B_{4}\left(V_{\Sigma_{4}}^{w}\right)_{0 \text {,throat }}$, with $B_{4}>1$, to parameterize the unknown bulk contribution. In the non-compact limit, $\left(V_{\Sigma_{4}}^{w}\right)_{0 \text {,throat }}$ diverges. Here we identify the leading order divergence and regularize the throat volume by introducing the UV cutoff $\hat{r}_{\mathrm{UV}}$, where the throat attaches to the bulk and $h\left(\hat{r}_{\mathrm{UV}}\right) \approx 1$. We use the following approximation to the warp factor $[15,25]$,

$$
h \approx \frac{L^{4}}{\hat{r}^{4}} \log \frac{\hat{r}}{\varepsilon^{2 / 3}}
$$

where

$$
2 T_{3} L^{4}=\frac{27}{16 \pi^{2}}\left(\frac{3 g_{s} M}{2 \pi K}\right) N, \quad \log Q_{0} \equiv \log \frac{\hat{r}_{\mathrm{UV}}}{\varepsilon^{2 / 3}} \approx \frac{2 \pi K}{3 g_{s} M} .
$$

At large radius $\hat{r}$ the D7-brane wraps an $S^{3}$ and the metric on the four-cycle is

$$
\mathrm{d} s_{\Sigma_{4}}^{2} \rightarrow \mathrm{d} \hat{r}^{2}+\hat{r}^{2} \mathrm{~d} s_{S^{3}}^{2}
$$

The warped four-cycle volume then becomes

$$
\begin{aligned}
\left(V_{\Sigma_{4}}^{w}\right)_{0} \equiv \int \mathrm{d}^{4} \xi \sqrt{g^{i n d}} h & =B_{4} L^{4} \operatorname{Vol}\left(S^{3}\right) \int_{\hat{r}_{\mu}}^{\hat{r}_{\mathrm{UV}}} \frac{\mathrm{d} \hat{r}}{\hat{r}} \log \frac{\hat{r}}{\varepsilon^{2 / 3}} \\
& =B_{4} L^{4} \operatorname{Vol}\left(S^{3}\right) \log Q_{\mu}\left[\log Q_{0}-\frac{1}{2} \log Q_{\mu}\right]
\end{aligned}
$$

where $Q_{\mu} \equiv \frac{\hat{r}_{\mathrm{UV}}}{\hat{r}_{\mu}}>1$. The numerical value of $\operatorname{Vol}\left(S^{3}\right)$ depends on the specific embedding. For the Kuperstein embedding we have [20]

$$
\operatorname{Vol}\left(S^{3}\right)=\frac{16 \pi^{2}}{9}
$$

and hence, by (B.7),

$$
\Gamma=\frac{3}{2 \pi} B_{4} N \log Q_{\mu}\left[1-\frac{1}{2} \frac{\log Q_{\mu}}{\log Q_{0}}\right]
$$

This implies

$$
\begin{aligned}
\omega_{0} \equiv a \sigma_{0} & =\frac{3}{2} B_{4}\left(\frac{N}{n}\right) \log Q_{\mu}\left[1-\frac{1}{2} \frac{\log Q_{\mu}}{\log Q_{0}}\right] \\
& \approx \frac{3}{2} B_{4}\left(\frac{N}{n}\right) \log Q_{\mu}
\end{aligned}
$$


Since, $B_{4}>1, N / n>1$ and $Q_{\mu}>1$, this can assume a range of values, with $\omega_{0}=\mathcal{O}(10)$ being easily achievable. The value of $\omega_{0}$ is important as it determines the scale of inflation

$$
\frac{V_{d S}}{M_{P}^{4}} \sim \frac{e^{-2 \omega_{0}}}{2 \omega_{0}} .
$$

In particular, for $B_{4}=\mathcal{O}(1)$ and $\log Q_{\mu}=\mathcal{O}(1)$, constraints on the minimal phenomenologically viable inflation scale, $V_{d S}>\mathcal{O}\left(\mathrm{TeV}^{4}\right) \sim 10^{-60} M_{P}^{4}$ or $\omega_{0} \lesssim 150$, translate into an upper limit on the background five form flux

$$
\frac{N}{n}<\mathcal{O}\left(10^{2}\right)
$$

This can be a serious constraint on nonperturbative volume stabilization by the KKLT mechanism.

Finally, the ACR [17] embeddings, $\prod_{i=1}^{4} w_{i}^{p_{i}}=\mu^{P}$, satisfy

$$
\operatorname{Vol}\left(S^{3}\right)=\frac{16 \pi^{2}}{9} P
$$

and hence

$$
\omega_{0} \approx \frac{3}{2} P B_{4}\left(\frac{N}{n}\right) \log Q_{\mu} .
$$

Possible corrections to these results coming from induced magnetic fields on the D7-branes are discussed in Appendix E. There we show that the result for the Kuperstein four-cycle is in fact unchanged, while ACR four-cycles may receive a correction. However, even for the ACR case this would only affect numerical details, and in any event this uncertainty in the precise ACR result may be absorbed in the uncertainty of the value of $B_{4}$.

\section{B.3 Canonical field range}

\section{B.3.1 Canonical inflaton}

The inflaton action includes the kinetic term

$$
\mathcal{L}_{\text {kin }}=-\frac{1}{2} T_{3} e^{-4 u(\hat{r})}\left(\partial_{\mu} \hat{r}\right)^{2} \equiv-\frac{1}{2}\left(\partial_{\mu} \varphi\right)^{2},
$$

so the canonical inflaton field is

$$
\varphi=\int\left(T_{3} \frac{\Gamma}{\tilde{\Gamma}(\hat{r})}\right)^{1 / 2} \mathrm{~d} \hat{r} .
$$

For analytical considerations the following approximation is often sufficient (see Appendix D for more accurate analytical results)

$$
\varphi^{2} \approx \frac{\Gamma}{\tilde{\Gamma}(\hat{r})} T_{3} \hat{r}^{2} \approx \frac{2 \sigma_{0}}{\rho+\bar{\rho}} T_{3} \hat{r}^{2} \approx T_{3} \hat{r}^{2} \equiv \phi^{2} .
$$


This is independent of the split into breathing mode and fiducial metric in (B.1):

$$
\frac{\varphi^{2}}{M_{P}^{2}}=\frac{\left[\Gamma e^{4 u}\right]}{\tilde{\Gamma}} \frac{T_{3}\left[e^{2 u} \hat{r}^{2}\right]}{\left[M_{P}^{2} e^{6 u}\right]}=\frac{\tilde{r}^{2}}{\frac{1}{\pi} T_{3} \widetilde{V}_{6}^{w}}
$$

This implies

$$
\frac{\gamma k}{\rho+\bar{\rho}}=\frac{\Gamma}{\rho+\bar{\rho}} \frac{T_{3} \hat{r}^{2}}{6 M_{P}^{2}} \approx \frac{\Gamma}{\tilde{\Gamma}} \frac{T_{3} \hat{r}^{2}}{6 M_{P}^{2}}=\frac{1}{6} \frac{\varphi^{2}}{M_{P}^{2}}
$$

and the DeWolfe-Giddings Kähler potential becomes separable

$$
\kappa^{2} \mathcal{K} \approx-3 \log (\rho+\bar{\rho})-3 \log u, \quad u \equiv 1-\frac{1}{6} \frac{\varphi^{2}}{M_{P}^{2}}
$$

\section{B.3.2 Bound on the field range}

We now derive the microscopic bound on the inflaton field range in four-dimensional Planck units [21]. Recall that the Planck mass depends on the warped volume of the internal space as

$$
M_{P}^{2}=\frac{1}{\pi}\left(T_{3}\right)^{2} V_{6}^{w}
$$

where $V_{6}^{w}$ is computed from the fiducial metric excluding the breathing mode. The warped volume of the internal space receives contributions both from the throat and from the bulk, $V_{6}^{w}=\left(V_{6}^{w}\right)_{\text {throat }}+\left(V_{6}^{w}\right)_{\text {bulk }}$. Since the bulk metric is not known we use the parametrization $V_{6}^{w} \equiv B_{6}\left(V_{6}^{w}\right)_{\text {throat }}$, with $B_{6}>1$, to characterize the unknown bulk contribution. Using the warp factor (B.20), we find that the leading contribution to the throat volume is

$$
\left(V_{6}^{w}\right)_{\text {throat }}=\frac{\pi}{T_{3}} \frac{N}{4} \hat{r}_{\mathrm{UV}}^{2}
$$

This implies that the range of the inflaton is bounded by [21]

$$
\frac{\varphi^{2}}{M_{P}^{2}} \leq \frac{\varphi_{\mathrm{UV}}^{2}}{M_{P}^{2}}=\frac{4}{e^{4 u\left(r_{\mathrm{UV}}\right) N B_{6}}}
$$

where

$$
e^{4 u\left(r_{\mathrm{UV}}\right)}=\frac{\sigma\left(r_{\mathrm{UV}}\right)}{\sigma(0)} .
$$

In [21] two of us (D.B. and L.M.) explained that this relation sharply limits the amount of gravitational waves that can be expected in D-brane inflation models. One of the main results of the present paper is that the bound (B.41) also provides a surprisingly strong constraint on the possibility of fine-tuning the inflaton potential, even in cases where the energy scale of inflation is too low for gravitational waves to be relevant. 
The bound (B.41) is written in a slightly stronger and more general form than the bound given in [21],

$$
\frac{\varphi^{2}}{M_{P}^{2}} \leq \frac{4}{N} .
$$

The factor $B_{6}>1$ is simply an explicit representation of the unknown bulk contribution to $V_{6}^{w}$ and hence to $M_{P}^{2}$. The factor of the breathing mode requires further explanation. Recall that we have used the convention $e^{u(0)}=1$, so that the breathing mode factor is unity when the D3-brane is at the tip of the throat. We will now argue that in the configurations of interest, fluctuations of the breathing mode as the D3-brane is displaced from the tip are characterized by the condition $e^{4 u(r)}=\frac{\sigma(r)}{\sigma(0)} \geq 1$. The D3-brane potential from moduli stabilization is minimized when the D3-brane is at the tip of the throat, in all the configurations studied in this paper (see also [43]). Displacing the D3-brane from the tip increases the potential, and so tends to increase the compactification volume, because positive energy causes a runaway potential for the volume. (We have confirmed this expectation by explicit numerical analysis in $\S 4.3$, and by analytical arguments in Appendix D.) Thus, moving the D3-brane up the throat dilates the space, and leads to $e^{4 u(r)}=\frac{\sigma(r)}{\sigma(0)}>1$ for $r>0$. This effect goes in the direction of making the bound (B.41) stronger than (B.43), but the effect is very small in practice: in the cases we considered, $1<\frac{\sigma\left(r_{\mathrm{UV}}\right)}{\sigma(0)}<1$.1. Such a factor is a negligible correction in comparison to the uncertainties in $B_{6}$, so it is very reasonable to omit it, as in $[21]$.

\section{Stability in the Angular Directions}

\section{C.1 Kuperstein embedding}

The following analysis complements our Kuperstein case study of $\S 3$ and $\S 4$. In $\S$ C.1.1 we derive the condition for extremal trajectories $z_{1}= \pm \frac{r^{3 / 2}}{\sqrt{2}}$ whose angular stability we investigate in $\S$ C.1.2.

\section{C.1.1 Extremal trajectories}

Recall that the Kuperstein potential (3.3) depends only on the following dynamical fields: $\left|z_{1}\right|^{2}, z_{1}+\overline{z_{1}}, r$, and $\sigma=\operatorname{Re}(\rho)$. Along a trajectory that extremizes the potential in the angular directions we must have $\frac{\partial V}{\partial \Psi_{i}}=0$ for all $r$, so we aim to find points in $T^{1,1}$ that satisfy

$$
\frac{\partial\left|z_{1}\right|^{2}}{\partial \Psi_{i}}=0=\frac{\partial\left(z_{1}+\overline{z_{1}}\right)}{\partial \Psi_{i}}
$$


We examine (C.1) by introducing local coordinates in the vicinity of a fiducial point $\mathbf{z}_{0} \equiv$ $\left(z_{1}^{\prime}, z_{2}^{\prime}, z_{3}^{\prime}, z_{4}^{\prime}\right)$. The coordinates around this point are given by the five generators of $S O(4)$ acting nontrivially on $\mathbf{z}_{0}$

$$
\mathbf{z}\left(r, \Psi_{i}\right)=\exp (\mathbf{T}) \mathbf{z}_{0} .
$$

The Kuperstein embedding, $z_{1}=\mu$, breaks the global $S O(4)$ symmetry of the conifold down to $S O(3)$, and the D3-brane potential preserves this $S O(3)$ symmetry. We will find that the actual trajectory breaks this $S O(3)$ down to $S O(2)$, which we take to act on $z_{3}$ and $z_{4}$. The coordinates that make this $S O(2)$ stability group manifest are given by

$$
\mathrm{T} \equiv\left(\begin{array}{cc|cc}
0 & \alpha_{2} & \alpha_{3} & \alpha_{4} \\
-\alpha_{2} & 0 & \beta_{3} & \beta_{4} \\
\hline-\alpha_{3} & -\beta_{3} & 0 & 0 \\
-\alpha_{4} & -\beta_{4} & 0 & 0
\end{array}\right),
$$

where $\Psi_{i} \equiv\left\{\alpha_{i}, \beta_{i}\right\} \in \mathbb{R}$ are the local coordinates of the base of the cone. We aim to find $\mathbf{z}_{0}$ such that the potential $V\left(z_{1}+\overline{z_{1}},\left|z_{1}\right|^{2}\right)$ is extremal along $\mathbf{z}_{0}$. We here find trajectories along which the linear variation of $z_{1}+\overline{z_{1}}$ and $\left|z_{1}\right|^{2}$ vanishes. First, we observe from (C.2) and (C.3) that for arbitrary $\mathbf{z}_{0}$ we have

$$
\delta z_{1}=\sum_{i=2}^{4} \alpha_{i} z_{i}^{\prime}, \quad \alpha_{i} \in \mathbb{R} .
$$

and, hence,

$$
\delta\left|z_{1}\right|^{2}=\sum_{i=2}^{4} \alpha_{i}\left(z_{i}^{\prime} \bar{z}_{1}^{\prime}+z_{1}^{\prime} \bar{z}_{i}^{\prime}\right) \equiv 0 .
$$

To satisfy (C.5) for all $\alpha_{i}$ one requires

$$
z_{i}^{\prime}=i \rho_{i} z_{1}^{\prime}, \quad \rho_{i} \in \mathbb{R}
$$

We may use $S O(3)$ to set $\rho_{3}=\rho_{4}=0$, while keeping $\rho_{2}$ finite. The conifold constraint, $z_{1}^{2}+z_{2}^{2}=0$, then implies $\rho_{2}= \pm 1$, while the requirement

$$
\delta\left(z_{1}^{\prime}+\bar{z}_{1}^{\prime}\right)=a_{2}\left(z_{2}^{\prime}+\bar{z}_{2}^{\prime}\right)=0
$$

makes $z_{2}^{\prime}$ purely imaginary and $z_{1}^{\prime}$ real. This proves that the following is an extremal trajectory of the brane potential for the Kuperstein potential

$$
z_{1}^{\prime}= \pm \frac{1}{\sqrt{2}} r^{3 / 2}, \quad z_{2}^{\prime}= \pm i z_{1}^{\prime} .
$$




\section{C.1.2 Stability}

Let us now study the stability of the potential along the path (C.8). From (C.2) one finds

$$
z_{1}=z_{1}^{\prime}\left[1-\frac{1}{2}\left(\alpha_{2}^{2}+\alpha_{3}^{2}+\alpha_{4}^{2}\right)+\frac{i}{2} \rho_{2}\left(2 \alpha_{2}-\alpha_{3} \beta_{3}-\alpha_{4} \beta_{4}\right)+\cdots\right],
$$

and

$$
\begin{aligned}
z_{1}+\bar{z}_{1} & =2 z_{1}^{\prime}\left[1-\frac{1}{2}\left(\alpha_{2}^{2}+\alpha_{3}^{2}+\alpha_{4}^{2}\right)+\cdots\right], \\
\left|z_{1}\right|^{2} & =\left(z_{1}^{\prime}\right)^{2}\left[1-\left(\alpha_{3}^{2}+\alpha_{4}^{2}\right)+\cdots\right] .
\end{aligned}
$$

Since the Kuperstein potential (3.3) depends only on $r, z_{1}+\overline{z_{1}}$, and $z_{1} \overline{z_{1}}$, and because

$$
\left.\frac{\partial\left|z_{1}\right|^{2}}{\partial \Psi_{i}}\right|_{0}=\left.\frac{\partial\left(z_{1}+\overline{z_{1}}\right)}{\partial \Psi_{i}}\right|_{0}=0
$$

where $\left.(\ldots)\right|_{0}$ denotes evaluation at $\mathbf{z}_{0}$, we find

$$
\left.\frac{\partial V}{\partial \Psi_{i}}\right|_{0}=0
$$

and

$$
\left.\frac{\partial^{2} V}{\partial \Psi_{i} \partial \Psi_{j}}\right|_{0}=\left.\left[\frac{\partial V}{\partial\left|z_{1}\right|^{2}} \frac{\partial^{2}\left|z_{1}\right|^{2}}{\partial \Psi_{i} \partial \Psi_{j}}+\frac{\partial V}{\partial\left(z_{1}+\overline{z_{1}}\right)} \frac{\partial^{2}\left(z_{1}+\overline{z_{1}}\right)}{\partial \Psi_{i} \partial \Psi_{j}}\right]\right|_{0},
$$

where

$$
\left.\partial_{i} \partial_{j}\left|z_{1}\right|^{2}\right|_{0}= \pm\left.\frac{r^{3 / 2}}{\sqrt{2}} \partial_{i} \partial_{j}\left(z_{1}+\overline{z_{1}}\right)\right|_{0}+r^{3} \delta_{i 2} \delta_{j 2}=-r^{3} \delta_{i 3} \delta_{j 3}-r^{3} \delta_{i 4} \delta_{j 4}
$$

Hence, the angular mass matrix at $\mathbf{z}_{0}$ has the form

$$
\left.\frac{\partial^{2} V}{\partial \Psi_{i} \partial \Psi_{j}}\right|_{0}=\left(\begin{array}{ccccc}
X & 0 & 0 & 0 & 0 \\
0 & X+Y & 0 & 0 & 0 \\
0 & 0 & X+Y & 0 & 0 \\
0 & 0 & 0 & 0 & 0 \\
0 & 0 & 0 & 0 & 0
\end{array}\right) \mid \begin{gathered}
\alpha_{2} \\
\alpha_{3} \\
\alpha_{4} \\
\beta_{3} \\
\beta_{4}
\end{gathered}
$$

where

$$
\begin{aligned}
X & \left.\equiv \mp \sqrt{2} r^{3 / 2} \frac{\partial V}{\partial\left(z_{1}+\overline{z_{1}}\right)}\right|_{0}, \\
Y & \equiv-\left.r^{3} \frac{\partial V}{\partial\left|z_{1}\right|^{2}}\right|_{0} .
\end{aligned}
$$


The flat directions in the $\beta_{3}, \beta_{4}$ angles parameterize the symmetry group $S O(3) / S O(2)$ that leaves the Kuperstein embedding $z_{1}=\mu$ invariant. The angles $\alpha_{3}$ and $\alpha_{4}$ have degenerate eigenvalues.

To calculate the eigenvalues $X$ and $Y$ we note that the F-term potential (3.3) may be written as

$$
\begin{aligned}
V_{F}= & \mathcal{C}(r, \sigma) \mathcal{G}^{1 / n}\left[(2 a \sigma+6)-\frac{6 e^{a \sigma}\left|W_{0}\right|}{\left|A_{0}\right|} \mathcal{G}^{-1 / 2 n}\right. \\
& \left.+\frac{3}{2 n}\left(\frac{1}{\mu}\left(z_{1}+\overline{z_{1}}\right)-\frac{2}{\mu^{2}}\left|z_{1}\right|^{2}\right) \mathcal{G}^{-1}+\frac{4 c}{n} \frac{r}{r_{\mu}}\left(1-\frac{\left|z_{1}\right|^{2}}{2 r^{3}}\right) \mathcal{G}^{-1}\right],
\end{aligned}
$$

and the D-term potential (2.20) depends only on $r$ and is independent of the angles (at least far from the tip [43]). In (C.19) we have defined the functions

$$
\begin{aligned}
\mathcal{C}(r, \sigma) & \equiv \frac{\kappa^{2} a\left|A_{0}\right|^{2} e^{-2 a \sigma}}{3 U^{2}}>0, \quad \partial_{z_{1}+\overline{z_{1}}} \mathcal{C}=\partial_{\left|z_{1}\right|^{2}} \mathcal{C}=0 \\
\mathcal{G} & \equiv \frac{|A|}{\left|A_{0}\right|}=\left|1-\frac{z_{1}}{\mu}\right|^{2}, \quad \partial_{z_{1}+\overline{z_{1}}} \mathcal{G}=-\frac{1}{\mu}, \quad \partial_{\left|z_{1}\right|^{2}} \mathcal{G}=\frac{1}{\mu^{2}}, \\
\mathcal{G}_{0} & \left.\equiv \mathcal{G}\right|_{0}=\left|1 \mp x^{3 / 2}\right|^{2}=g(x)^{2},
\end{aligned}
$$

and the variable

$$
x \equiv \frac{r}{r_{\mu}}=\frac{\phi}{\phi_{\mu}} .
$$

After a lengthy but straightforward computation we find

$$
\begin{aligned}
& X= \pm \frac{2 \mathcal{C}}{n} \frac{x^{3 / 2}}{\left|1 \mp x^{3 / 2}\right|^{2(1-1 / n)}}\left[2 a \sigma+\frac{9}{2}-\frac{3 e^{a \sigma}\left|W_{0}\right|}{\left|A_{0}\right|} \frac{1}{\left|1 \mp x^{3 / 2}\right|^{1 / n}}\right. \\
& \left.\mp 3\left(1-\frac{1}{n}\right) \frac{x^{3 / 2}\left(1 \mp x^{3 / 2}\right)}{\left|1 \mp x^{3 / 2}\right|^{2}}-3 c\left(1-\frac{1}{n}\right) \frac{x}{\left|1 \mp x^{3 / 2}\right|^{2}}\right] \text {, }
\end{aligned}
$$

and

$$
X+Y=\left(1 \mp x^{3 / 2}\right) X+\frac{2 \mathcal{C} c}{n} \frac{x}{\left|1 \mp x^{3 / 2}\right|^{2(1-1 / n)}}\left(1+\frac{3}{2 c} x^{2}\right)
$$

where

$$
c \equiv \frac{9}{4 n a \sigma_{0} \frac{\phi_{\mu}^{2}}{M_{P}^{2}}} .
$$

Notice that stability of the trajectory $z_{1}= \pm \frac{1}{\sqrt{2}} r^{3 / 2}$ for both positive and negative real $z_{1}$ only requires that $X>0$ (from (C.25) this automatically implies $X+Y>0$, at least for the regime of interest, $r<r_{\mu}$ ). Hence, from equation (C.24) a simple numerical check can decide whether a specific scenario is stable in the angular directions. For all potential inflationary trajectories we have performed this stability test. 


\section{Analytical approximation}

In $\S 3$ and Appendix D we explain how the position of the Kähler modulus in the AdS vacuum, $\sigma_{F}$, shifts to $\sigma_{0}$ when the minimum gets uplifted to dS,

$$
a \sigma_{0} \approx a \sigma_{F}+\frac{s}{a \sigma_{F}},
$$

where $\frac{3\left|W_{0}\right|}{\left|A_{0}\right|} e^{a \sigma_{F}}=2 a \sigma_{F}+3$ and $\frac{3\left|W_{0}\right|}{\left|A_{0}\right|} e^{a \sigma_{0}} \approx 2 a \sigma_{0}+3+2 s$. Substituting this into (C.24) we find

$$
\begin{aligned}
X= \pm \frac{2 \mathcal{C}}{n} \frac{x^{3 / 2}}{\left|1 \mp x^{3 / 2}\right|^{2(1-1 / n)}} & {\left[\frac{3}{2}-\frac{2 s}{\left|1 \mp x^{3 / 2}\right|^{1 / n}}+2 a \sigma_{0}\left(1-\frac{1}{\left|1 \mp x^{3 / 2}\right|^{1 / n}}\right)\right.} \\
& +3\left(1-\frac{1}{\left|1 \mp x^{3 / 2}\right|^{1 / n}}\right) \mp 3\left(1-\frac{1}{n}\right) \frac{x^{3 / 2}\left(1 \mp x^{3 / 2}\right)}{\left|1 \mp x^{3 / 2}\right|^{2}} \\
& \left.-3 c\left(1-\frac{1}{n}\right) \frac{x}{\left|1 \mp x^{3 / 2}\right|^{2}}\right] .
\end{aligned}
$$

In the limit $x \rightarrow 0$ this becomes

$$
\lim _{x \rightarrow 0} X=\mp \frac{\mathcal{C}}{n} x^{3 / 2}[4 s-3] .
$$

Hence, the near-tip region is stable on the negative axis and unstable on the positive axis. Notice that it was essential to include the shift from uplifting to realize this.

\section{C.2 Ouyang embedding}

\section{C.2.1 Extremal trajectories}

For the Ouyang embedding, $w_{1}=\mu$, the brane potential depends on $w_{1}+\overline{w_{1}},\left|w_{1}\right|^{2}$ and $\left|w_{2}\right|^{2}$. To find extremal trajectories of the potential we therefore require

$$
\frac{\partial\left|w_{1}\right|^{2}}{\partial \Psi_{i}}=\frac{\partial\left|w_{2}\right|^{2}}{\partial \Psi_{i}}=\frac{\partial\left(w_{1}+\overline{w_{1}}\right)}{\partial \Psi_{i}}=0 .
$$

We introduce local coordinates by applying generators of $S U(2)$ to the generic point $W_{0}$

$$
W=e^{i \boldsymbol{\top}_{1}} W_{0} e^{-i \boldsymbol{\top}_{2}}, \quad W_{0} \equiv\left(\begin{array}{cc}
w_{3}^{\prime} & w_{2}^{\prime} \\
w_{1}^{\prime} & w_{4}^{\prime}
\end{array}\right)
$$

where

$$
\mathrm{T}_{i} \equiv\left(\begin{array}{cc}
\alpha_{i} & \beta_{i}+i \gamma_{i} \\
\beta_{i}-i \gamma_{i} & -\alpha_{i}
\end{array}\right)
$$


This implies

$$
\delta w_{1}=-i\left(\alpha_{1}+\alpha_{2}\right) w_{1}^{\prime}+\left(-\beta_{1}+i \gamma_{1}\right) w_{3}^{\prime}+\left(\beta_{2}-i \gamma_{2}\right) w_{4}^{\prime}+\cdots
$$

and $\delta\left(w_{1}+\bar{w}_{1}\right)=0$ gives $w_{1}^{\prime} \in \mathbb{R}, w_{3}^{\prime}=w_{4}^{\prime}=0$. We find that $\delta\left|w_{1}\right|^{2}=0$ and $\delta\left|w_{2}\right|^{2}=0$ if $w_{2}^{\prime} \in \mathbb{R}$. The conifold constraint $w_{1}^{\prime} w_{2}^{\prime}=0$ then restricts the solution to the following two options:

$$
w_{1}^{\prime}=0, \quad\left|w_{2}^{\prime}\right|=r^{3 / 2}, \quad \Leftrightarrow \quad \theta_{1}=\theta_{2}=0
$$

or

$$
w_{1}^{\prime}= \pm r^{3 / 2}, \quad w_{2}^{\prime}=0, \quad \Leftrightarrow \quad \theta_{1}=\theta_{2}=\pi
$$

\section{Delta-flat direction}

For $w_{1}^{\prime}=0$ the superpotential correction to the potential vanishes and inflation is impossible, as noted in [18] and reviewed in $\S 5$.

\section{Non-delta-flat direction}

For $w_{2}^{\prime}=0$ the superpotential correction to the potential does not vanish. In fact, along this extremal trajectory the potential can be shown to be identical to the potential for the Kuperstein case. However, angular stability for the Ouyang and Kuperstein cases is different, as we now discuss.

\section{C.2.2 Stability}

Delta-flat direction

Near $w_{1}^{\prime}=0, w_{2}^{\prime} \neq 0$ we have

$$
\begin{aligned}
& w_{1}=w_{2}^{\prime}\left[\beta_{1} \beta_{2}-\gamma_{1} \gamma_{2}-i\left(\beta_{1} \gamma_{2}+\beta_{2} \gamma_{1}\right)\right] \\
& w_{2}=w_{2}^{\prime}\left[1+i\left(\alpha_{1}+\alpha_{2}\right)-\frac{1}{2}\left(\alpha_{1}+\alpha_{2}\right)^{2}-\frac{1}{2}\left(\beta_{1}^{2}+\beta_{2}^{2}+\gamma_{1}^{2}+\gamma_{2}^{2}\right)\right]
\end{aligned}
$$

and (to second order)

$$
\begin{aligned}
w_{1}+\overline{w_{1}} & =2 w_{2}^{\prime}\left[\beta_{1} \beta_{2}-\gamma_{1} \gamma_{2}\right] \\
\left|w_{1}\right|^{2} & =0 \\
\left|w_{2}\right|^{2} & =\left(w_{2}^{\prime}\right)^{2}\left[1-\left(\beta_{1}^{2}+\beta_{2}^{2}+\gamma_{1}^{2}+\gamma_{2}^{2}\right)\right] .
\end{aligned}
$$


The mass matrix in these coordinates in non-diagonal, but may easily be diagonalized by the transformation

$$
\beta_{1,2}=\frac{u_{1} \pm v_{1}}{\sqrt{2}}, \quad \gamma_{1,2}=\frac{v_{2} \pm u_{2}}{\sqrt{2}}, \quad U^{2} \equiv u_{1}^{2}+u_{2}^{2}, \quad V^{2} \equiv v_{1}^{2}+v_{2}^{2} .
$$

This gives

$$
\begin{aligned}
w_{1}+\overline{w_{1}} & =w_{2}^{\prime}\left[U^{2}-V^{2}\right] \\
\left|w_{2}\right|^{2} & =\left(w_{2}^{\prime}\right)^{2}\left[1-\left(U^{2}+V^{2}\right)\right] .
\end{aligned}
$$

A lengthy but straightforward computation gives the eigenvalues of the angular mass matrix of the potential along the delta-flat direction

$$
\begin{aligned}
& X_{U}=\frac{2 \mathcal{C} c}{n} x\left[1 \mp \frac{1}{4 c} x^{1 / 2}\left(4 a \sigma+9-\frac{6 e^{a \sigma}\left|W_{0}\right|}{\left|A_{0}\right|}\right)\right], \\
& X_{V}=\frac{2 \mathcal{C} c}{n} x\left[1 \pm \frac{1}{4 c} x^{1 / 2}\left(4 a \sigma+9-\frac{6 e^{a \sigma}\left|W_{0}\right|}{\left|A_{0}\right|}\right)\right] .
\end{aligned}
$$

We have confirmed that these stability criteria are precisely what was found for the deltaflat direction in [18] ( $c f$. their equation (3.15)). Equation (C.44) and (C.45) show that the delta-flat direction is angularly stable if $x<x_{c}$ and unstable if $x>x_{c}$, where

$$
x_{c} \equiv\left(\frac{4 c}{4 s-3}\right)^{2}=\frac{1}{(4 s-3)^{2}}\left(\frac{9}{a \sigma_{0}}\right)^{2} \frac{1}{n^{2}} \frac{M_{P}^{4}}{\phi_{\mu}^{4}} .
$$

Equation (C.46) is the generalization of (5.21) to general $n$. Applying the field range bound in the form $\frac{\phi_{\mu}^{2}}{M_{P}^{2}}<\frac{4}{N}$ one finds

$$
x_{c}>\frac{1}{(4 s-3)^{2}}\left(\frac{9}{a \sigma_{0}}\right)^{2} \frac{N^{2}}{(4 n)^{2}} \geq 1 .
$$

For typical parameters the delta-flat direction is hence stable from the tip to at least the location of the D7-branes.

\section{Non-delta-flat direction}

The non-delta-flat trajectory of the Ouyang embedding is very closely related to the extremal trajectories of the Kuperstein embedding. In fact, the shape of the potential is identical for the two cases. However, the stability analysis reveals subtle, but important differences.

Near $w_{2}^{\prime}=0, w_{1}^{\prime} \neq 0$ we have

$$
\begin{aligned}
w_{1}+\overline{w_{1}} & =2 w_{1}^{\prime}\left[1-\frac{1}{2}\left(U^{2}+V^{2}\right)\right], \\
\left|w_{1}\right|^{2} & =\left(w_{1}^{\prime}\right)^{2}\left[1-V^{2}\right],
\end{aligned}
$$


where we have defined

$$
\begin{aligned}
U^{2} & \equiv\left(\alpha_{1}+\alpha_{2}\right)^{2} \\
V^{2} & \equiv \beta_{1}^{2}+\beta_{2}^{2}+\gamma_{1}^{2}+\gamma_{2}^{2} .
\end{aligned}
$$

Computing the eigenvalues of the angular mass matrix we find results that are almost identical to the Kuperstein results (C.24) and (C.25), except for one crucial sign difference:

$$
\begin{aligned}
& X_{U}=X, \\
& X_{V}=\left(1 \mp x^{3 / 2}\right) X+\frac{2 \mathcal{C} c}{n} \frac{x}{\left|1 \mp x^{3 / 2}\right|^{2(1-1 / n)}}\left(-1+\frac{3}{2 c} x^{2}\right),
\end{aligned}
$$

where $X$ is the Kuperstein result (C.24). Since the leading term in (C.53) now comes with the opposite sign ( $c f$. (C.25)), the non-delta-flat trajectory for Ouyang is typically unstable for small $x$ whereas the corresponding trajectory for Kuperstein is stable,

$$
X_{V} \approx-\frac{2 \mathcal{C} c}{n} \frac{x}{\left|1 \mp x^{3 / 2}\right|^{2(1-1 / n)}}<0 .
$$

This is consistent with the results of [18].

\section{C.3 Stability for small $r$}

To summarize our discussion of stability for the Kuperstein scenario and the Ouyang scenario we now give an intuitive explanation of angular stability in the limit of small $r$.

For either the Kuperstein or the Ouyang embedding, stability near the tip $r \rightarrow 0$ is controlled by the term in the potential proportional to $k^{1 \overline{1}}$. We first focus on the Kuperstein embedding. Since $k^{1 \overline{1}}$ contains a term proportional to $r^{-3}$, its contribution to the second derivative of the potential with respect to an angular variable $\Psi_{i}, \frac{\partial^{2} V}{\partial \Psi_{i}^{2}}$, grows as $r$. All other terms grow at least as $r^{3 / 2}$ (this follows from $\frac{\partial}{\partial \Psi}=\frac{\partial z_{i}}{\partial \Psi} \frac{\partial}{\partial z_{i}}+$ c.c. and $\frac{\partial z_{i}}{\partial \Psi} \sim r^{3 / 2}$ ). A parallel consideration confirms that $\hat{k}_{w}^{1 \overline{1}}$ is responsible for the leading contribution to the stability analysis in the case of the Ouyang embedding as well.

Now, the trajectories $z_{1}= \pm \frac{r^{3 / 2}}{\sqrt{2}}$ maximize $\left|z_{1}\right|^{2}$ for a given $r$, and any variation of angles may only increase $k^{1 \overline{1}}=r\left(1-\frac{\left|z_{1}\right|^{2}}{2 r^{3}}\right)$. Hence the trajectories in question are stable at small $r$ under fluctuations of any angles that affect $\left|z_{1}\right|^{2}$. So far, this analysis does not include the phase of $z_{1}$, which of course leaves $\left|z_{1}\right|^{2}$ invariant. The leading correction to the potential from fluctuations of this phase comes not through $k^{1 \overline{1}}$ but through terms in $V$ proportional to $z_{1}+\overline{z_{1}}$. These terms change sign when $z_{1}$ does; thus, one of the signs

in $z_{1}= \pm \frac{r^{3 / 2}}{\sqrt{2}}$ corresponds to the stable trajectory, while the other sign corresponds to an unstable trajectory. We showed above that the stable trajectory in fact corresponds to 
$z_{1}<0$ (careful consideration of the shift of the volume, $c f$. Appendix D, is required for that argument.)

The analysis for the Ouyang embedding is very similar. The delta-flat trajectory $\left|w_{2}\right|^{2}=$ $r^{3}\left(\theta_{1}=\theta_{2}=0\right)$ maximizes the ratio $\frac{\left|w_{2}\right|^{2}}{r^{3}}$. Thus, any angular fluctuation can only decrease the ratio $\frac{\left|w_{2}\right|^{2}}{r^{3}}$, without affecting $\frac{\left|w_{1}\right|^{2}}{r^{3}}$ to second order (cf. (C.38)). This is easily checked with the help of the angular coordinates $\theta_{i}$ (A.9). On the other hand, the trajectory $w_{1}= \pm r^{3 / 2}$ $\left(\theta_{1}=\theta_{2}=\pi\right)$ maximizes $\frac{\left|w_{1}\right|^{2}}{r^{3}}$, and angular fluctuations away from this trajectory decrease the ratio $\frac{\left|w_{1}\right|^{2}}{r^{3}}$, without affecting $\frac{\left|w_{2}\right|^{2}}{r^{3}}$ to second order. As a result, $\hat{k}_{w}^{1 \overline{1}}=r\left(1+\frac{\left|w_{1}\right|^{2}}{2 r^{3}}-\frac{\left|w_{2}\right|^{2}}{r^{3}}\right)$ cannot decrease in the case of the delta-flat trajectory $\left|w_{2}\right|^{2}=r^{3}$, but necessarily has a negative mode along the non-delta-flat trajectory $w_{1}= \pm r^{3 / 2}$. Hence, the non-delta-flat trajectory is unstable for small $r$. No further consideration is needed to show that the deltaflat trajectory $\left|w_{2}\right|^{2}=r^{3}$ is stable. Since angular fluctuations around $w_{1}=0$ cannot affect the term involving $w_{1}+\overline{w_{1}}$, the leading contribution always comes from $\hat{k}_{w}^{1 \overline{1}}$.

We have therefore demonstrated that near the tip, the trajectory $z_{1}=-\frac{r^{3 / 2}}{\sqrt{2}}$ is stable for the Kuperstein embedding, whereas the trajectory $w_{1}= \pm r^{3 / 2}$ in the Ouyang embedding is unstable.

\section{C.4 Higher-degree ACR embeddings}

We conclude this Appendix with a derivation of the non-delta flat trajectory for higher degree ACR embeddings. As before, to find an extremal radial direction we need to satisfy the equations $\frac{\partial V}{\partial \Psi_{i}}=0$ for any $r$. Since the potential for a general ACR embedding depends only on $|\Phi|^{2 P}, \operatorname{Re}\left(\Phi^{P}\right)$ and

$$
\begin{aligned}
Z \equiv & \left|p_{1} \frac{w_{4}}{w_{1}}+p_{3} \frac{w_{2}}{w_{3}}\right|^{2}+\left|p_{1} \frac{w_{3}}{w_{1}}+p_{4} \frac{w_{2}}{w_{4}}\right|^{2} \\
& +\left|p_{2} \frac{w_{3}}{w_{2}}+p_{4} \frac{w_{1}}{w_{4}}\right|^{2}+\left|p_{2} \frac{w_{4}}{w_{2}}+p_{3} \frac{w_{1}}{w_{3}}\right|^{2},
\end{aligned}
$$

we will consider these terms separately. Extremizing $\operatorname{Re}\left(\Phi^{P}\right)$ with respect to the phase of $\Phi$ selects real $\Phi^{P}$. For any non-zero $\Phi$ we can use equations (C.31) and (C.32) to show that

$$
\operatorname{Re}\left[\frac{\delta \Phi^{P}}{\Phi^{P}}\right]=\operatorname{Re}\left[\sum_{i} p_{i} \frac{\delta w_{i}}{w_{i}}\right]=0
$$

for

$$
\begin{aligned}
& \left|\frac{w_{1}}{w_{3}}\right|^{2}=\tan ^{2} \frac{\theta_{1}}{2}=\frac{p_{1}+p_{4}}{p_{2}+p_{3}} \\
& \left|\frac{w_{1}}{w_{4}}\right|^{2}=\tan ^{2} \frac{\theta_{2}}{2}=\frac{p_{1}+p_{3}}{p_{2}+p_{4}},
\end{aligned}
$$


and

$$
\Phi^{P}= \pm \lambda^{P} r^{3 P / 2}
$$

where

$$
\lambda^{P} \equiv \frac{\left(p_{1}+p_{3}\right)^{\frac{1}{2}\left(p_{1}+p_{3}\right)}\left(p_{1}+p_{4}\right)^{\frac{1}{2}\left(p_{1}+p_{4}\right)}\left(p_{2}+p_{3}\right)^{\frac{1}{2}\left(p_{2}+p_{3}\right)}\left(p_{2}+p_{4}\right)^{\frac{1}{2}\left(p_{2}+p_{4}\right)}}{P^{P}} .
$$

Notice that this point trivially extremizes $|\Phi|^{2 P}$ since

$$
\delta|\Phi|^{2 P}=|\Phi|^{2 P} \operatorname{Re}\left[\frac{\delta \Phi^{P}}{\Phi^{P}}\right]=0 .
$$

In addition, since $\Phi^{P}$ is real, equation (C.56) also implies $\delta \operatorname{Re}\left(\Phi^{P}\right)=0$. Finally, surprisingly enough, (C.57, C.58) also extremizes (C.55) with respect to the angular directions. Therefore (C.59) is an extremal radial trajectory which exists for a generic case in addition to the delta-flat direction, $\Phi=0$. For $P=1$ this reproduces the non-delta-flat direction for the Ouyang embedding, $c f$. Appendix C.2. For $P=2, p_{1}=p_{2}=1$ we find that the Karch-Katz embedding has a non-delta-flat direction given by $\Phi^{2}=w_{1} w_{2}=w_{3} w_{4}= \pm \frac{1}{4} r^{3}$.

Although every ACR embedding contains non-delta flat directions (C.59), we now argue that these higher degree embeddings $(P>1)$ are not promising settings for flat potentials. Recall that for the $P=1$ Kuperstein an inflection point could be arranged along $z_{1} \in \mathbb{R}^{-}$ (modulo consistency with microscopic constraints), basically because an $x^{3 / 2}$ term in the potential led to a negative divergence of $\eta$ for small $x$, which could be balanced against the $x^{2}$ term familiar from the $\eta$-problem for intermediate $x$ (see $\S 4.1$ ). For $P>1$ embeddings this possibility disappears, since there are then no terms of order lower than quadratic in $x$. Hence,

$$
\lim _{x \rightarrow 0} \eta=\frac{2}{3} .
$$

This is suggestive evidence that fine tuning in higher degree ACR embeddings cannot give inflation, at least not at $x<1$ (cf. [33]). 


\section{Stabilization of the Volume}

In the AdS minimum of a KKLT compactification, the stabilized value of the Kähler modulus $\omega_{F} \equiv a \sigma_{F}$ is given by the SUSY condition

$$
\left.D_{\rho} W\right|_{r=0, \omega_{F}}=\left.0 \quad \Rightarrow \quad \frac{\partial V_{F}}{\partial \omega}\right|_{\omega_{F}}=0,
$$

which in terms of the flux superpotential is [16]

$$
3 \frac{\left|W_{0}\right|}{\left|A_{0}\right|} e^{\omega_{F}}=2 \omega_{F}+3 .
$$

Adding an antibrane to lift the KKLT AdS minimum to a dS minimum induces a small shift in the stabilized volume, $\omega_{0}=\omega_{F}+\delta \omega$. We compute this shift in $\S$ D.1. This gives the value of the Kähler modulus in the absence of a mobile D3-brane (or when the brane is near the tip of the throat). The presence of a D3-brane away from the tip induces a further shift of the volume that depends on the brane position, which we denote $\omega_{\star}(r)$. We compute this dependence of the Kähler modulus on the D3-brane position in §D.2. (See also [33].)

\section{D.1 Shift induced by uplifting}

The stabilized value of the Kähler after uplifting, $\omega_{0}=\omega_{F}+\delta \omega$, is determined from

$$
\left.\frac{\partial V}{\partial \omega}\right|_{\omega_{0}}=\left.0 \approx \frac{\partial^{2} V_{F}}{\partial \omega^{2}}\right|_{\omega_{F}} \delta \omega+\left.\frac{\partial V_{D}}{\partial \omega}\right|_{\omega_{0}},
$$

where, from (2.20),

$$
\left.\frac{\partial V_{D}}{\partial \omega}\right|_{\omega_{0}}=\left.\left.\frac{-2 V_{D}}{\omega}\right|_{\omega_{0}} \approx \frac{-2 V_{D}}{\omega_{F}}\right|_{\omega_{F}}\left[1-3 \frac{\delta \omega}{\omega_{F}}\right] .
$$

Solving (D.3) and (D.4) for $\delta \omega$ we find

$$
\delta \omega=\frac{\omega_{F}}{3+\left.\frac{\omega^{2}}{2 V_{D}} \frac{\partial^{2} V_{F}}{\partial \omega^{2}}\right|_{\omega_{F}}} .
$$

From equation (3.11) we have

$$
\begin{aligned}
V_{F}(0, \omega) & =C \frac{e^{-2 \omega}}{(2 \omega)^{2}}\left[(2 \omega+6)-6 e^{\omega} \frac{\left|W_{0}\right|}{\left|A_{0}\right|}\right] \\
V_{D}(0, \omega) & =\left(D+D_{\text {other }}\right) \frac{1}{(2 \omega)^{2}}
\end{aligned}
$$


and

$$
\frac{\partial V_{F}}{\partial \omega}=-\frac{\omega+2}{\omega}\left[V_{F}+C \frac{e^{-2 \omega}}{2 \omega}\right] .
$$

Since

$$
V_{F}\left(0, \omega_{F}\right)=-C \frac{e^{-2 \omega_{F}}}{2 \omega_{F}},
$$

equation (D.8) vanishes at $\omega_{F}$, confirming equation (D.1). The second derivative of the F-term potential at $\omega_{F}$ is

$$
\begin{aligned}
\left.\frac{\partial^{2} V_{F}}{\partial \omega^{2}}\right|_{\omega_{F}} & =-\frac{2}{\left(\omega_{F}\right)^{2}}\left[\left(\omega_{F}\right)^{2}+\frac{5}{2} \omega_{F}+1\right] V_{F}\left(0, \omega_{F}\right) \\
& \approx+2\left|V_{F}\left(0, \omega_{F}\right)\right|
\end{aligned}
$$

where $\omega_{F} \gg 1$. Recalling the definition (3.19) and using $\frac{\left(\omega_{F}\right)^{2}}{s} \gg 1$ in equation (D.5), we find

$$
\omega_{0} \approx \omega_{F}+\frac{s}{\omega_{F}}
$$

Although $\delta \omega$ is small, it appears in an exponent in the potential (3.11), so that its effect there has to be considered:

$$
\begin{aligned}
3 \frac{\left|W_{0}\right|}{\left|A_{0}\right|} e^{\omega_{0}}=3 \frac{\left|W_{0}\right|}{\left|A_{0}\right|} e^{\omega_{F}} e^{\delta \omega} & \approx\left(2 \omega_{F}+3\right)\left(1+\frac{s}{\omega_{F}}\right) \\
& \approx 2 \omega_{F}+3+2 s, \\
& \approx 2 \omega_{0}+3+2 s .
\end{aligned}
$$

\section{D.2 Shift induced by brane motion}

Adding a mobile D3-brane to the compactification induces a further shift of the Kähler modulus that depends on the radial position of the brane, $\omega_{\star}(r)$. The function $\omega_{\star}(r)$ is determined by the solution of a transcendental equation,

$$
\left.\partial_{\omega} V\right|_{\omega_{\star}(r)}=0
$$

Although equation (D.13) does not have an exact analytic solution, here we derive a simple approximate solution. (See also [33].) The precise form of the solution we give here is only valid for the trajectory $z_{1}=-\frac{r^{3 / 2}}{\sqrt{2}}$ of the Kuperstein potential (see $\S 2$ ). While these results can easily be generalized to the trajectory $z_{1}=\frac{r^{3 / 2}}{\sqrt{2}}$ and to more general embeddings, we have argued in the main body of the paper that the trajectory $z_{1}=-\frac{r^{3 / 2}}{\sqrt{2}}$ in the Kuperstein embedding is of primary interest as far as the possibility of inflationary solutions is concerned. 
First, we notice that $\omega_{\star}$ appears both in polynomial terms and exponential terms in (D.13). Letting $\omega_{\star} \rightarrow \omega_{0}=\omega_{F}+\frac{s}{\omega_{F}}$ (see equation (D.11)) in all polynomial terms, but not in the exponentials, we transform the transcendental equation (D.13) into a quadratic equation for $e^{\omega_{\star}(r)}$. Solving this we obtain the dependence of the Kähler modulus on the brane position as an expansion in $x$

$$
\omega_{\star}(r)=\omega_{0}\left[1+c_{1} x+c_{3 / 2} x^{3 / 2}+c_{2} x^{2}+\ldots\right],
$$

where

$$
\omega_{0}=\omega_{F}+\frac{s}{\omega_{F}}+\frac{s(3 s-5)}{2 \omega_{F}^{2}}+\mathcal{O}\left(\omega_{F}^{-3}\right),
$$

and

$$
\begin{aligned}
c_{1} & =\left(\frac{27}{8 n^{2}} \frac{M_{P}^{2}}{\phi_{\mu}^{2}}\right) \frac{1}{\omega_{F}^{3}}+\mathcal{O}\left(\omega_{F}^{-4}\right), \\
c_{3 / 2} & =\frac{1}{n} \frac{1}{\omega_{F}}\left[1-\frac{1}{2 \omega_{F}}\right]+\mathcal{O}\left(\omega_{F}^{-3}\right), \\
c_{2} & =\left(\frac{s-1}{6} \frac{\phi_{\mu}^{2}}{M_{P}^{2}}\right) \frac{1}{\omega_{F}^{2}}+\mathcal{O}\left(\omega_{F}^{-3}\right) .
\end{aligned}
$$

As we can see from this, typically $c_{3 / 2} \gg c_{1}, c_{2}$, so that the following approximation can be tried

$$
\omega_{\star}(r) \approx \omega_{0}\left[1+c_{3 / 2} x^{3 / 2}\right] .
$$

Notice also that $c_{1}, c_{3 / 2}, c_{2}$ are all positive - the volume shrinks as the D3-brane moves towards the tip. Fig. 6 shows the D3-brane potential derived from (D.20) (dashed line) and the exact numerical result (solid line). Notice that the analytic approximation is always steeper than the more precise numerical result and therefore systematically underestimates the total number of $e$-folds [34].

\section{D.3 Canonical inflaton revisited}

Equipped with (D.19) for the evolution of the volume modulus, we may obtain a more accurate analytical result for the canonical inflaton field (B.34),

$$
\varphi(r)=\int\left(\frac{3 \sigma_{0} T_{3}}{U\left(r, \sigma_{\star}(r)\right)}\right)^{1 / 2} \mathrm{~d} r,
$$

where

$$
\frac{3 \sigma_{0} T_{3}}{U\left(r, \sigma_{\star}(r)\right)}=\frac{3}{2} T_{3}\left(\frac{\omega_{\star}(r)}{\omega_{\star}(0)}-\frac{\frac{3}{2} T_{3} r^{2}}{6 M_{P}^{2}}\right)^{-1}
$$



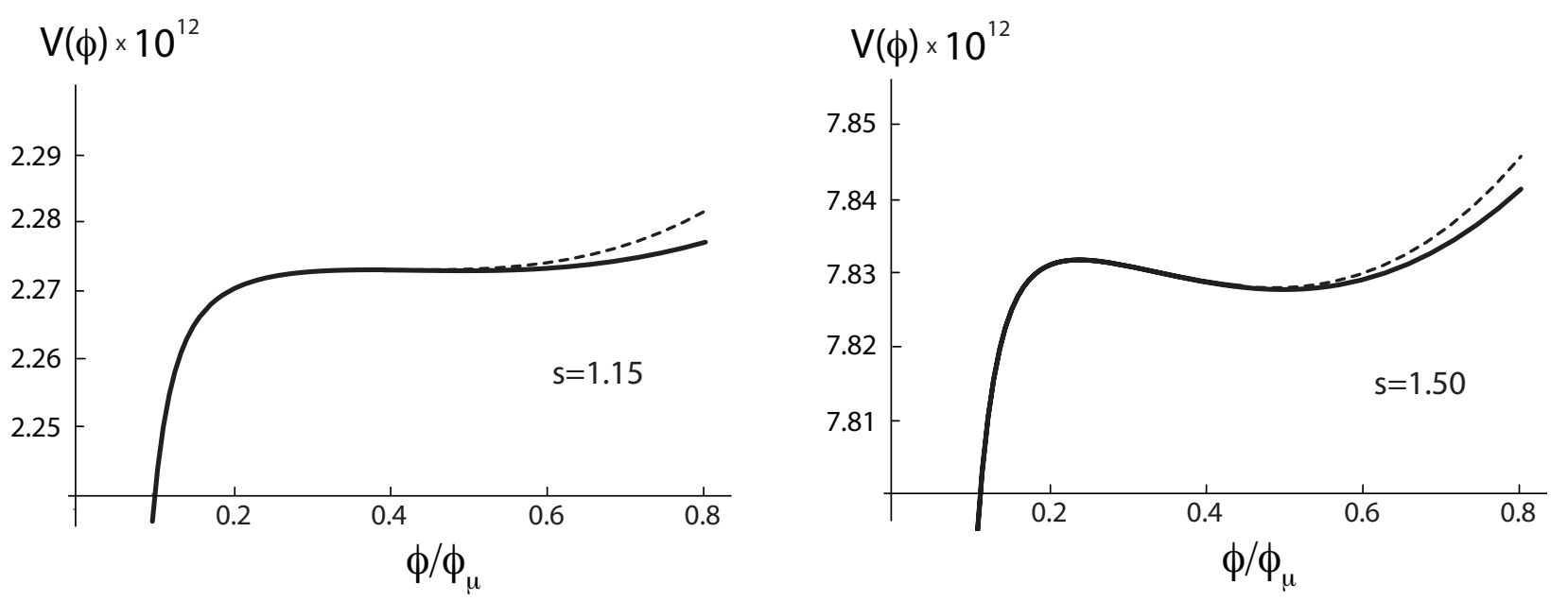

Figure 6: D3-brane potential $\mathbb{V}(\phi)$.

Shown are the analytic potential derived from (D.19)(dashed line) and the exact numerical result (solid line).

This implies

$$
\begin{aligned}
\varphi(r) & =\int\left[1+c_{3 / 2}\left(\frac{\phi}{\phi_{\mu}}\right)^{3 / 2}-\frac{\phi^{2}}{6 M_{P}^{2}}\right]^{-1 / 2} \mathrm{~d} \phi \\
& \approx \phi\left[1-\frac{c_{3 / 2}}{5}\left(\frac{\phi}{\phi_{\mu}}\right)^{3 / 2}+\frac{\phi^{2}}{36 M_{P}^{2}}\right], \\
& \approx \phi .
\end{aligned}
$$




\section{E Effects of Induced Magnetic Fields}

One may be concerned that magnetic fields induced on the wrapped D7-branes could give rise to an additional contribution to the D3-brane potential. In this appendix we address this problem and show that if supersymmetry is broken by a brane-antibrane pair in a (conformally) Calabi-Yau throat, the induced magnetic fields do not affect our considerations.

Let us consider a D7-brane with the worldvolume action $S_{D_{7}}=S_{\mathrm{DBI}}+S_{\mathrm{CS}}$,

$$
S_{D_{7}}=-T_{7} \int_{\mathcal{M}_{8}} \mathrm{~d}^{8} \xi e^{-\Phi} \sqrt{-\operatorname{det}\left(\hat{G}_{a b}+\hat{B}_{a b}+2 \pi \alpha^{\prime} F_{a b}\right)}-T_{7} \int_{\mathcal{M}_{8}} \sum_{q} \hat{C}_{[q]} \wedge e^{\hat{B}_{[2]}+2 \pi \alpha^{\prime} F},
$$

where hats denote pullbacks of bulk fields onto the worldvolume of the D7-brane, $C_{[p]}$ are the RR potentials, and $F$ is the worldvolume gauge field strength. The indices $a, b$ run through the eight dimensions of the D7-brane worldvolume, while Greek indices $\mu, \nu$ and Roman indices $i, j$ will denote directions along Minkowski spacetime and along the internal Calabi-Yau space, respectively. Let the warped background metric be

$$
\mathrm{d} s^{2}=h^{-1 / 2} \eta_{\mu \nu} \mathrm{d} x^{\mu} \mathrm{d} x^{\mu}+h^{1 / 2} \sum_{i=1}^{6} G_{i}^{2}
$$

where $G_{i}$ are six-dimensional vielbeins. We assume that the D7-brane wraps some four-cycle $\Sigma_{4}$ in the compact space, and that there is a magnetic field $B$ in the bulk and an induced gauge field $F$ on the D7-brane. The gauge field can be decomposed into components $\mathcal{F}$ in the internal dimensions and components $\mathbb{F}$ in spacetime. We absorb a dimensionful coefficient, $2 \pi \alpha^{\prime}$, in the definition of $\mathcal{F}$ and $\mathbb{F}$. The action of the D7-brane depends on $\mathcal{F}$ through the combination $M \equiv \hat{B}+\mathcal{F}$. We are interested in the Born-Infeld and Chern-Simons actions for the D7-brane in the presence of a D3-brane and a non-trivial gauge field $\mathbb{F}$ in the spacetime directions. Let us first consider the D7-brane alone, without any D3-branes. We do not yet assume that the compactification manifold is conformally Calabi-Yau. The kappa-symmetry condition for a supersymmetric embedding of the D7-brane can be reformulated purely in geometrical terms without use of the $\Gamma$-algebra [44]. First, supersymmetry requires the fourcycle $\Sigma_{4}$ to be holomorphic. Then the following identities for the forms on $\Sigma_{4}$ should be satisfied

$$
h^{1 / 2} \hat{J} \wedge M=\tanh \theta\left(\frac{h}{2} \hat{J} \wedge \hat{J}-\frac{1}{2} M \wedge M\right), \quad M^{2,0}=0 .
$$

Here $J$ is a (pseudo)-Kähler form of the internal manifold $J=\frac{i}{2} \sum_{i=1}^{3} G_{2 i-1} \wedge G_{2 i}$ and the angle $\theta$ characterizes the geometry of the supergravity solution as explained in [44]. For the warped Calabi-Yau case of the warped deformed conifold [14] solution, $\theta=0$, while the solutions on the baryonic branch [45] satisfy $\cosh \theta=e^{-\Phi}$, where $\Phi$ is the dilaton. We use 
the block-diagonal form of the matrices in (E.1) to evaluate the DBI action

$$
\operatorname{det}\left(\hat{G}_{a b}+\hat{B}_{a b}+2 \pi \alpha^{\prime} F_{a b}\right)=\operatorname{det}\left(h^{-1 / 2} \eta_{\mu \nu}+\mathbb{F}_{\mu \nu}\right) \times \operatorname{det}\left(h^{1 / 2} \hat{G}_{i j}+M_{i j}\right),
$$

and express the answer as a power series in $\mathbb{F}$

$$
\sqrt{-\operatorname{det}\left(h^{-1 / 2} \eta+\mathbb{F}\right)}=\left[h^{-1}-\frac{1}{4} \operatorname{Tr} \mathbb{F}^{2}+\mathcal{O}\left(\mathbb{F}^{4}\right)\right] .
$$

Since $\hat{G}_{i j}$ (and $\hat{J}$ ) has only $(1,1)$ components, equation (E.3) implies

$$
\operatorname{det}\left(h^{1 / 2} \hat{G}+M\right)=\left(h \operatorname{det}^{1 / 2} \hat{G}-\operatorname{Pf} M\right)^{2}+h(\hat{J} \wedge M)_{1234}^{2},
$$

where $\operatorname{Pf} X$ of a 2 -form $X$ is defined as $\operatorname{Pf} X=\frac{1}{2}(X \wedge X)_{1234}$. Expression (E.6) is algebraic because $\operatorname{det} \hat{G}$ also factorizes: $\operatorname{Pf} \hat{J}=\operatorname{det}^{1 / 2} \hat{G}$. Combining (E.6) with (E.3) we get

$$
\operatorname{det}\left(h^{1 / 2} \hat{G}+M\right)=\left(h \operatorname{det}^{1 / 2} \hat{G}-\operatorname{Pf} M\right)^{2}\left(1+\tanh ^{2} \theta\right),
$$

and

$$
S_{\mathrm{DBI}}=-T_{7} \int_{\mathcal{M}_{8}} \mathrm{~d}^{8} \xi e^{-\Phi}\left[h^{-1}-\frac{1}{4} \operatorname{Tr} \mathbb{F}^{2}+\mathcal{O}\left(\mathbb{F}^{4}\right)\right]\left(h \operatorname{det}^{1 / 2} \hat{G}-\operatorname{Pf} M\right) \frac{1}{\cosh \theta} .
$$

From now on we focus on the warped deformed conifold solution, although the calculation below is the same for any background with imaginary self-dual flux. Since the dilaton is constant, $\Phi=\theta=0$, the leading terms of the $\mathbb{F}$-expansion are

$$
\begin{aligned}
S_{\mathrm{DBI}} & =-\frac{1}{2} T_{7} \operatorname{Vol}_{4} \int_{\Sigma}\left(\hat{J} \wedge \hat{J}-h^{-1} M \wedge M\right)+ \\
& +T_{7} \int_{\mathbb{R}^{1,3}} \mathrm{~d}^{4} x \operatorname{Tr} \mathbb{F}^{2} \int_{\Sigma_{4}}\left(h \operatorname{det}^{1 / 2} \hat{G} \mathrm{~d}^{4} \xi-\frac{1}{2} M \wedge M\right)+\mathcal{O}\left(\mathbb{F}^{4}\right) .
\end{aligned}
$$

To compute the Chern-Simons term, we note that the only relevant RR flux in the warped deformed conifold case is

$$
C_{4}=h^{-1} \mathrm{Vol}_{4},
$$

where $\operatorname{Vol}_{4}=\mathrm{d} x^{1} \wedge \cdots \wedge \mathrm{d} x^{4}$ is a volume form in the Minkowski space and $\left.e^{\hat{B}+2 \pi \alpha^{\prime} F}\right|_{\Sigma_{4}}=$ $\left.e^{M+\mathbb{F}}\right|_{\Sigma_{4}}=\left.\frac{1}{2} M \wedge M\right|_{\Sigma_{4}}$. Hence,

$$
S_{\mathrm{CS}}=-\frac{1}{2} T_{7} \operatorname{Vol}_{4} \int_{\Sigma_{4}} h^{-1} M \wedge M .
$$


Adding the DBI and CS actions, we have

$$
\begin{aligned}
S_{D_{7}} & =-\frac{1}{2} T_{7} \operatorname{Vol}_{4} \int_{\Sigma_{4}} \hat{J} \wedge \hat{J}+ \\
& +T_{7} \int_{\mathbb{R}^{1,3}} \mathrm{~d}^{4} x \operatorname{Tr} \mathbb{F}^{2} \int_{\Sigma_{4}}\left(h \operatorname{det}^{1 / 2} \hat{G} \mathrm{~d}^{4} \xi-\frac{1}{2} M \wedge M\right)+\mathcal{O}\left(\mathbb{F}^{4}\right) .
\end{aligned}
$$

Finally, we are ready to add the D3-brane to our considerations. As was discussed in [9], to leading order the D3-brane only changes the warp factor $h$, but does not affect the metric $G$ or the RR or NSNS forms. The $h$ (and $M$ ) dependence of the leading term of the DBI action (E.9) is precisely cancelled by the CS term (E.11). Since the leading term in (E.12) is independent of $h$, it is not affected by the D3-brane.

For the solutions in question the Kähler form $J$ is closed. Therefore the leading term in (E.12), $\int_{\Sigma_{4}} \hat{J} \wedge \hat{J}$, does not depend on $\Sigma_{4}$ itself but only on its homology class. This also means that the D7-brane tension does not vary under small perturbations of $\Sigma_{4}$, as expected for the tension of supersymmetric branes. This is only true when $\Sigma_{4}$ is compact. In the non-compact case this term diverges as the fourth power of a cut-off radius $r_{\mathrm{UV}}^{4}$.

In the subleading term in (E.12) the warp factor $h$ and magnetic field $M$ do not mix. The first term is proportional to $h$ and is responsible for the factor in the nonperturbative superpotential discussed in detail in [9]. For the validity of the considerations of [9] it is important that this term is independent of $M$. The second term involves $M$, but is $h$ independent. To leading order it is not affected by the backreaction of the D3-brane.

\section{E.1 Kuperstein embedding}

After deriving the general formula (E.12) we are ready to apply it to the particular case of the Kuperstein embedding. We are interested in calculating the unperturbed value of the gauge coupling

$$
\frac{1}{g_{0}^{2}}=2 T_{7} \int_{\Sigma_{4}}\left(h \operatorname{det}^{1 / 2} \hat{G} \mathrm{~d}^{4} \xi-\frac{1}{2} M \wedge M\right)
$$

before the D3-brane is added. In $\S \mathrm{B} .2$ we computed the warped four-cycle volume (the first term in (E.13)), but ignored the effects of induced magnetic fields (the second term in (E.13)). Here we justify this approximation. For the Kuperstein embedding this is a straightforward calculation, since in this case $F=0$ and hence $M=\hat{B}$ [20]. The integral (E.13) is divergent in the non-compact case. To estimate its lower limit in the compact case we evaluate its dependence on the cutoff parameter $r_{\mathrm{UV}}$, defined by $h\left(r_{\mathrm{UV}}\right) \approx 1$. For large $r_{\mathrm{UV}}$ we use the KT [15] approximation for the metric (2.5) and the NSNS fields [15] and 
drop all corrections in $1 / r$. In this limit we find

$$
-\frac{1}{2} B \wedge B=\frac{9}{4} \log (r)^{2} \mathrm{~d} \theta_{1} \wedge \sin \theta_{1} \mathrm{~d} \phi_{1} \wedge \mathrm{d} \theta_{2} \wedge \sin \theta_{2} \mathrm{~d} \phi_{2}
$$

In the large $r$ region, where the conifold can be approximated by a cone over $T^{1,1}$, the four-cycle $\Sigma_{4}$ specified by the Kuperstein embedding $z_{1}=\mu$ approaches a cone over an $S^{3}$ given by

$$
\phi_{2}=\phi_{1}, \quad \theta_{2}=\theta_{1}
$$

We choose the variables $\phi_{1}, \theta_{1}, \psi$ and $r$ to parameterize the internal part of the D7-brane world-volume $\Sigma_{4}$ with $\theta_{2}, \phi_{2}$ given by (E.15). It is clear from (E.15) that (E.14) gives no contribution in the large $r$ limit. Therefore its contribution is finite when $r_{\mathrm{UV}} \rightarrow \infty$ and is small compared to the divergent warped four-cycle volume computed in (B.2) if $r_{\mathrm{UV}}$ is large enough. Hence the contribution of the magnetic field in (E.13) is negligible for the Kuperstein embedding.

\section{E.2 ACR embeddings}

For the case of the general ACR embedding (5.1), we note that in the large $r$ region, where $\Sigma_{4}$ can be approximated by a cone over a certain three-sphere (or a sum of several threespheres), (E.14) vanishes as well. This follows immediately from the equations specifying the base of the cone,

$$
\theta_{i}=0, \pi
$$

This is not sufficient to say that the second term in (E.13) gives no contribution, because the gauge field $F$ is not zero in this case. Nevertheless one would not expect the contribution of the induced gauge field to be dominant in the DBI action, as the value of the induced gauge field is such that the DBI action is minimized. Therefore the second term in (E.13) can only change the coefficient in front of the leading divergence in (B.32) but will not change the power of the divergence itself. 


\section{F Symbols Used in the Paper}

Table 1: Definitions of symbols used in this paper

\begin{tabular}{|c|c|c|}
\hline Variable & Description & Definition \\
\hline$z_{i}$ & complex conifold coordinates & $\sum_{i} z_{i}^{2}=0,(\mathrm{~A} .5)-(\mathrm{A} .8)$ \\
\hline$w_{i}$ & complex conifold coordinates & $w_{1} w_{2}-w_{3} w_{4}=0,(\mathrm{~A} .9)-(\mathrm{A} .12)$ \\
\hline$\varepsilon$ & conifold deformation parameter & $\sum_{i} z_{i}^{2}=\varepsilon$, equation $(2.1)$ \\
\hline$r$ & radial coordinate on the conifold & $r^{3}=\sum_{i}\left|z_{i}\right|^{2}$ \\
\hline$\hat{r}$ & radial coordinate on the conifold & $\hat{r}^{2}=\frac{3}{2} r^{2}, \mathrm{~d} s^{2}=\mathrm{d} \hat{r}^{2}+\ldots$ \\
\hline$\tilde{r}$ & radial coordinate on the conifold & $\tilde{r}=e^{u} \hat{r}$ \\
\hline$e^{u}$ & breathing mode & equation (B.1) \\
\hline$g_{\alpha \bar{\beta}}$ & fiducial metric & \\
\hline$\tilde{g}_{\alpha \bar{\beta}}$ & physical metric & $\tilde{g}_{\alpha \bar{\beta}}=e^{2 u} g_{\alpha \bar{\beta}}$ \\
\hline$k$ & Kähler potential & $g_{\alpha \bar{\beta}}=k_{, \alpha \bar{\beta}}, k=\frac{3}{2}\left(\sum_{i} z_{i}^{2}\right)^{2 / 3}$ \\
\hline$\phi_{i}, \theta_{i}, \psi$ & angular coordinates on $T^{1,1}$ & equations (A.5)-(A.8) \\
\hline$h(r)$ & warp factor & equation (2.4) \\
\hline$r_{\mathrm{UV}}$ & radius at the UV end & $\ln r_{\mathrm{UV}} / \varepsilon^{2 / 3}=2 \pi K /\left(3 g_{s} M\right)$ \\
\hline$\phi$ & inflaton field & $\phi^{2}=T_{3} \hat{r}^{2}$ \\
\hline$\varphi$ & canonical inflaton field & Appendix B, $\varphi^{2} \approx \frac{\sigma_{\star}(0)}{\sigma(\phi)} \phi^{2}$ \\
\hline $\mathcal{K}$ & Kähler potential & $\kappa^{2} \mathcal{K}=-3 \log U$ \\
\hline$W$ & superpotential & \\
\hline$U$ & argument of Kähler potential [29] & $U=\rho+\bar{\rho}-\gamma k$ \\
\hline$\rho$ & complex Kähler modulus & \\
\hline$\sigma$ & real part of $\rho$ & $2 \sigma=\rho+\bar{\rho}$ \\
\hline$\tau$ & imaginary part of $\rho$ & $2 i \tau=\rho-\bar{\rho}$ \\
\hline$\sigma_{\star}$ & stabilized volume modulus & $\left.\partial_{\sigma} V\right|_{\sigma_{\star}}=0$ \\
\hline$\omega_{\star}$ & rescaled volume modulus & $\omega_{\star} \equiv a \sigma_{\star}$ \\
\hline$W_{0}$ & GVW-flux superpotential & $W_{0}=\int G \wedge \Omega$ \\
\hline$W_{\mathrm{np}}$ & non-perturbative superpotential & $W_{\mathrm{np}}=A\left(z_{i}\right) e^{-a \rho}$ \\
\hline$f\left(z_{i}\right)$ & embedding equation & $A\left(z_{i}\right) \propto\left(f\left(z_{i}\right)\right)^{1 / n}$ \\
\hline$g\left(z_{i}\right)$ & embedding equation & $g\left(z_{i}\right)=f\left(z_{i}\right) / f(0)$ \\
\hline$A_{0}$ & prefactor of $W_{\mathrm{np}}$ & $A_{0}=A\left(z_{i}=0\right)$ \\
\hline$V_{F}$ & F-term potential & equation (2.8) \\
\hline$V_{D}$ & D-term potential & $V_{D}=D(r) U^{-2}(\rho, r) ;$ equation $(2.20)$ \\
\hline$D$ & scale of D-term energy & $D \equiv 2 h_{0}^{-1} T_{3}$ \\
\hline
\end{tabular}


Table 2: Definitions of symbols used in this paper (continued)

\begin{tabular}{|c|c|c|}
\hline Variable & Description & Definition \\
\hline$\epsilon, \eta$ & slow-roll parameters & 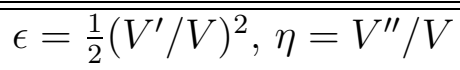 \\
\hline$g_{s}$ & string coupling & \\
\hline$T_{3}$ & D3-brane tension & $T_{3}^{-1}=(2 \pi)^{3} g_{s}\left(\alpha^{\prime}\right)^{2}$ \\
\hline$\mu$ & embedding parameter & $z_{1}=\mu$ \\
\hline$n$ & \# of embedded D7's & \\
\hline$a$ & parameter in $W_{\mathrm{np}}$ & $a=2 \pi / n$ \\
\hline$r_{\mu}$ & minimal radius of $\mathrm{D} 7$ & $r_{\mu}^{3}=2 \mu^{2}$ \\
\hline$\phi_{\mu}$ & rescaled $r_{\mu}$ & $\phi_{\mu}^{2}=\frac{3}{2} T_{3} r_{\mu}^{2}$ \\
\hline$M, K$ & flux on the $A$ and $B$ cycle & \\
\hline$N$ & five-form flux & $N \equiv M K$ \\
\hline$L$ & AdS radius & equation (B.21) \\
\hline$M_{P}$ & 4d Planck mass & $M_{P}^{2}=\frac{1}{\pi}\left(T_{3}\right)^{2} V_{6}^{w}$ \\
\hline$V_{6}^{w}$ & warped 6-volume & \\
\hline$V_{\Sigma_{4}}^{w}$ & warped 4-cycle volume & Appendix B \\
\hline$s$ & ratio of D- and F-term & equation (3.19) \\
\hline$x$ & ratio of $r$ and $r_{\mu}$ & $x=r / r_{\mu}$ \\
\hline$x_{0}$ & location of $\eta=0$ & \\
\hline$Q_{\mu}$ & ratio of $r_{\mathrm{UV}}$ and $r_{\mu}$ & $Q_{\mu}=r_{\mathrm{UV}} / r_{\mu}>1$ \\
\hline$B_{6}$ & bulk contribution to $V_{6}^{w}$ & $\left(V_{6}^{w}\right)_{\text {bulk }}=B_{6}\left(V_{6}^{w}\right)_{\text {throat }}$ \\
\hline$B_{4}$ & bulk contribution to $V_{\Sigma_{4}}^{w}$ & $\left(V_{\Sigma_{4}}^{w}\right)_{\text {bulk }}=B_{4}\left(V_{\Sigma_{4}}^{w}\right)_{\text {throat }}$ \\
\hline$\omega_{F}$ & Kähler modulus before uplifting & equation (D.2) \\
\hline$\omega_{0}$ & Kähler modulus after uplifting & $\omega_{0} \approx \omega_{F}+s / \omega_{F}$ \\
\hline$\Gamma$ & factor in $\mathcal{K}$ & $\Gamma=2 \sigma_{0}$ \\
\hline$\tilde{\Gamma}$ & factor in $\mathcal{K}$ & $\tilde{\Gamma} \equiv \Gamma e^{4 u}=U$ \\
\hline$\gamma$ & prefactor in the Kähler potential & $\gamma=\frac{\Gamma}{6} \frac{T_{3}}{M_{P}^{2}}=\frac{\sigma_{0}}{3} \frac{T_{3}}{M_{P}^{2}}$ \\
\hline$c$ & factor in $V_{F}$ & $c^{-1}=4 \pi \gamma r_{\mu}^{2}$ \\
\hline$c_{3 / 2}$ & factor in volume shift & equation (3.22) \\
\hline$X, X+Y$ & eigenvalues of Hessian & Appendix C \\
\hline P & degree of ACR embeddings & $\prod_{i} w_{i}^{p_{i}}=\mu^{P}$ \\
\hline$p_{i}$ & embedding parameter & $p_{i} \in \mathbb{Z}$ \\
\hline$\Phi$ & collective coordinate for ACR & $\Phi^{P} \equiv \prod_{i} w_{i}^{p_{i}}$ \\
\hline
\end{tabular}




\section{References}

[1] D. Baumann, A. Dymarsky, I. R. Klebanov, L. McAllister and P. J. Steinhardt, "A Delicate Universe," arXiv:0705.3837 [hep-th].

[2] A. H. Guth, "The Inflationary Universe: A Possible Solution To The Horizon And Flatness Problems," Phys. Rev. D 23, 347 (1981);

A. D. Linde, "A New Inflationary Universe Scenario: A Possible Solution Of The Horizon, Flatness, Homogeneity, Isotropy And Primordial Monopole Problems," Phys. Lett. B 108, 389 (1982);

A. Albrecht and P. J. Steinhardt, "Cosmology For Grand Unified Theories With Radiatively Induced Symmetry Breaking," Phys. Rev. Lett. 48, 1220 (1982).

[3] D. N. Spergel et al. [WMAP Collaboration], "First Year Wilkinson Microwave Anisotropy Probe (WMAP) Observations: Determination of Cosmological Parameters," Astrophys. J. Suppl. 148, 175 (2003) [arXiv:astro-ph/0302209];

H. V. Peiris et al., "First year Wilkinson Microwave Anisotropy Probe (WMAP) observations: Implications for inflation," Astrophys. J. Suppl. 148, 213 (2003) [arXiv:astro-ph/0302225];

D. N. Spergel et al., "Wilkinson Microwave Anisotropy Probe (WMAP) three year results: Implications for cosmology," [arXiv:astro-ph/0603449].

[4] G. R. Dvali and S. H. H. Tye, "Brane inflation," Phys. Lett. B 450, 72 (1999) [arXiv:hep-ph/9812483].

[5] S. Alexander, "Inflation from D - Anti-D-Brane Annihilation," Phys. Rev. D 65, 023507 (2002) [arXiv:hep-th/0105032];

G. Dvali, Q. Shafi and S. Solganik, "D-brane Inflation," arXiv:hep-th/0105203;

C. P. Burgess, M. Majumdar, D. Nolte, F. Quevedo, G. Rajesh and R. J. Zhang, "The Inflationary Brane-Antibrane Universe," JHEP 07, 047 (2001) [arXiv:hep-th/0105204];

D. Choudhury, D. Ghoshal, D. P. Jatkar and S. Panda, "Hybrid inflation and brane-antibrane system," JCAP 0307, 009 (2003) [arXiv:hep-th/0305104].

[6] S. Kachru, R. Kallosh, A. Linde, J. Maldacena, L. McAllister and S. P. Trivedi, "Towards inflation in string theory," JCAP 0310, 013 (2003) [arXiv:hep-th/0308055].

[7] S. Dimopoulos, S. Kachru, J. McGreevy and J. G. Wacker, "N-flation," arXiv:hep-th/0507205;

J. P. Conlon and F. Quevedo, "Kähler moduli inflation," JHEP 0601, 146 (2006) [arXiv:hep-th/0509012]; 
J. J. Blanco-Pillado et al., "Inflating in a better racetrack," JHEP 0609, 002 (2006) [arXiv:hep-th/0603129];

J. R. Bond, L. Kofman, S. Prokushkin and P. M. Vaudrevange, "Roulette inflation with Kähler moduli and their axions," arXiv:hep-th/0612197.

[8] A. Linde, "Inflation and string cosmology," eConf C040802, L024 (2004) [J. Phys. Conf. Ser. 24, 151 (2005 PTPSA,163,295-322.2006)] [arXiv:hep-th/0503195];

S. H. Henry Tye, "Brane inflation: String theory viewed from the cosmos," arXiv:hep-th/0610221;

J. M. Cline, "String cosmology," arXiv:hep-th/0612129;

R. Kallosh, "On Inflation in String Theory," arXiv:hep-th/0702059.

[9] D. Baumann, A. Dymarsky, I. R. Klebanov, J. Maldacena, L. McAllister and A. Murugan, "On D3-brane potentials in compactifications with fluxes and wrapped D-branes," JHEP 0611, 031 (2006) [arXiv:hep-th/0607050].

[10] O. J. Ganor, "A note on zeroes of superpotentials in F-theory," Nucl. Phys. B 499, 55 (1997) [arXiv:hep-th/9612077].

[11] M. Berg, M. Haack and B. Kors, "Loop corrections to volume moduli and inflation in string theory," Phys. Rev. D 71, 026005 (2005) [arXiv:hep-th/0404087].

[12] S. B. Giddings and A. Maharana, "Dynamics of warped compactifications and the shape of the warped landscape," Phys. Rev. D 73, 126003 (2006) [arXiv:hep-th/0507158].

[13] H. Firouzjahi and S. H. H. Tye, "Closer towards inflation in string theory," Phys. Lett. B 584, 147 (2004) [arXiv:hep-th/0312020].

[14] I. R. Klebanov and M. J. Strassler, "Supergravity and a confining gauge theory: Duality cascades and chiSB-resolution of naked singularities," JHEP 0008, 052 (2000) [arXiv:hep-th/0007191].

[15] I. R. Klebanov and A. A. Tseytlin, "Gravity duals of supersymmetric $S U(N) \times S U(N+M)$ gauge theories," Nucl. Phys. B 578, 123 (2000) [arXiv:hep-th/0002159].

[16] S. Kachru, R. Kallosh, A. Linde and S. P. Trivedi, "De Sitter vacua in string theory," Phys. Rev. D 68, 046005 (2003) [arXiv:hep-th/0301240].

[17] D. Areán, D. E. Crooks and A. V. Ramallo, "Supersymmetric probes on the conifold," JHEP 0411, 035 (2004) [arXiv:hep-th/0408210].

[18] C. P. Burgess, J. M. Cline, K. Dasgupta and H. Firouzjahi, "Uplifting and inflation with D3 branes," JHEP 0703, 027 (2007) [arXiv:hep-th/0610320]. 
[19] P. Ouyang, "Holomorphic D7-branes and flavored $N=1$ gauge theories," Nucl. Phys. B 699, 207 (2004) [arXiv:hep-th/0311084].

[20] S. Kuperstein, "Meson spectroscopy from holomorphic probes on the warped deformed conifold," JHEP 0503, 014 (2005) [arXiv:hep-th/0411097].

[21] D. Baumann and L. McAllister, "A microscopic limit on gravitational waves from D-brane inflation," arXiv:hep-th/0610285.

[22] S. B. Giddings, S. Kachru and J. Polchinski, "Hierarchies from fluxes in string compactifications," Phys. Rev. D 66, 106006 (2002) [arXiv:hep-th/0105097].

[23] M. R. Douglas and S. Kachru, "Flux compactification," arXiv:hep-th/0610102.

[24] P. Candelas and X. C. de la Ossa, "Comments on conifolds," Nucl. Phys. B 342, 246 (1990).

[25] C. P. Herzog, I. R. Klebanov and P. Ouyang, "Remarks on the warped deformed conifold," arXiv:hep-th/0108101; "D-branes on the conifold and $N=1$ gauge / gravity dualities," arXiv:hep-th/0205100.

[26] D. Lüst, P. Mayr, S. Reffert and S. Stieberger, "F-theory flux, destabilization of orientifolds and soft terms on D7-branes," Nucl. Phys. B 732, 243 (2006) [arXiv:hep-th/0501139].

[27] L. Görlich, S. Kachru, P. K. Tripathy and S. P. Trivedi, "Gaugino condensation and non-perturbative superpotentials in flux compactifications," JHEP 0412, 074 (2004) [arXiv:hep-th/0407130].

[28] S. Kachru, J. McGreevy and P. Svrcek, "Bounds on masses of bulk fields in string compactifications," JHEP 0604, 023 (2006) [arXiv:hep-th/0601111].

[29] O. DeWolfe and S. B. Giddings, "Scales and hierarchies in warped compactifications and brane worlds," Phys. Rev. D 67, 066008 (2003) [arXiv:hep-th/0208123].

[30] C. P. Burgess, P. G. Camara, S. P. de Alwis, S. B. Giddings, A. Maharana, F. Quevedo and K. Suruliz, "Warped supersymmetry breaking," arXiv:hep-th/0610255.

[31] S. Gukov, C. Vafa and E. Witten, "CFT's from Calabi-Yau four-folds," Nucl. Phys. B 584, 69 (2000) [Erratum-ibid. B 608, 477 (2001)] [arXiv:hep-th/9906070].

[32] A. Dymarsky, I. R. Klebanov and N. Seiberg, "On the moduli space of the cascading $S U(M+p) \times S U(p)$ gauge theory," JHEP 0601, 155 (2006) [arXiv:hep-th/0511254].

[33] A. Krause and E. Pajer, "Chasing Brane Inflation in String-Theory," arXiv:0705.4682 [hep-th]. 
[34] S. Panda, M. Sami and S. Tsujikawa, "Prospects of inflation in delicate D-brane cosmology," arXiv:0707.2848 [hep-th].

[35] R. Holman, P. Ramond and G. G. Ross, "Supersymmetric Inflationary Cosmology," Phys. Lett. B 137, 343 (1984);

D. H. Lyth and A. Riotto, "Particle physics models of inflation and the cosmological density perturbation," Phys. Rept. 314, 1 (1999) [arXiv:hep-ph/9807278].

[36] R. Allahverdi, K. Enqvist, J. Garcia-Bellido and A. Mazumdar, "Gauge invariant MSSM inflaton," Phys. Rev. Lett. 97, 191304 (2006) [arXiv:hep-ph/0605035];

R. Allahverdi, K. Enqvist, J. Garcia-Bellido, A. Jokinen and A. Mazumdar, "MSSM flat direction inflation: Slow roll, stability, fine tunning and reheating," JCAP 0706, 019 (2007) [arXiv:hep-ph/0610134];

J. C. Bueno Sanchez, K. Dimopoulos and D. H. Lyth, "A-term inflation and the MSSM," JCAP 0701, 015 (2007) [arXiv:hep-ph/0608299].

[37] N. Itzhaki and E. D. Kovetz, "Inflection Point Inflation and Time Dependent Potentials in String Theory," arXiv:0708.2798 [hep-th]; see also N. Itzhaki's talk at the Conference "String Theory: Achievements and Perspectives," Jerusalem and Tel-Aviv April 2007, http://stringfest.tau.ac.il

[38] A. Karch and E. Katz, "Adding flavor to AdS/CFT," JHEP 0206, 043 (2002) [arXiv:hep-th/0205236].

[39] M. Berg, M. Haack and B. Körs, "String loop corrections to Kähler potentials in orientifolds," JHEP 0511, 030 (2005) [arXiv:hep-th/0508043].

[40] M. Berg, M. Haack and B. Körs, "On volume stabilization by quantum corrections," Phys. Rev. Lett. 96, 021601 (2006) [arXiv:hep-th/0508171].

[41] K. Becker, M. Becker, M. Haack and J. Louis, "Supersymmetry breaking and alpha'-corrections to flux induced potentials," JHEP 0206, 060 (2002) [arXiv:hep-th/0204254].

[42] V. Balasubramanian, P. Berglund, J. P. Conlon and F. Quevedo, "Systematics of moduli stabilisation in Calabi-Yau flux compactifications," JHEP 0503, 007 (2005) [arXiv:hep-th/0502058].

[43] O. DeWolfe, L. McAllister, G. Shiu and B. Underwood, "D3-brane vacua in stabilized compactifications," arXiv:hep-th/0703088.

[44] M. Marino, R. Minasian, G. W. Moore and A. Strominger, "Nonlinear instantons from supersymmetric p-branes," JHEP 0001, 005 (2000) [arXiv:hep-th/9911206]. 
[45] A. Butti, M. Grana, R. Minasian, M. Petrini and A. Zaffaroni, "The baryonic branch of Klebanov-Strassler solution: A supersymmetric family of $S U(3)$ structure backgrounds," JHEP 0503, 069 (2005) [arXiv:hep-th/0412187]. 